\title{
Convergent synthesis of trisubstituted tetrahydrofurans via bis- thermally reactive 1,5-diene-tert-butyl carbonates.
}

Fabien Emmetiere and Alexander J. Grenning*

Department of Chemistry, University of Florida, PO Box 117200

E-mail: grenning@ufl.edu

Abstract: Cascade reactions (also known as domino- or tandem reactions) are an efficient strategy for generating molecular complexity. We report that synergizing the thermal reactivity of 3,3-dicyano-1,5-dienes and tert-butyl carbonates result in stereospecific 2,3,4-trisubstituted tetrahydrofuran synthesis. While substituted tetrahydrofurans can be challenging to synthesize, this discovery converts readily available 1,5dienes derived from aldehydes, malononitrile, and cis-buten-1,4-diol into complex tetrahydrofurans via a process involving thermal Cope rearrangement, Boc-deprotection, and oxy-Michael addition. Described herein includes background related to the discovery, optimization and scope, and representative functional group interconversion chemistry for the scaffolds.

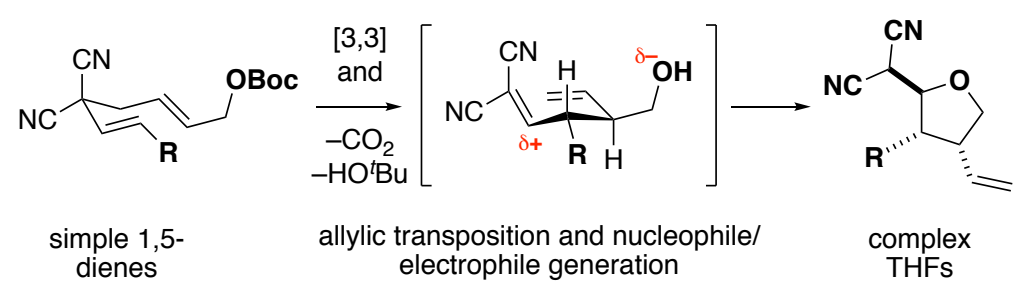

\section{Introduction}

Substituted tetrahydrofurans (THFs) are a common heterocyclic core found in diverse, bioactive natural products such as terpenes, (anethofuran,${ }^{[1]}$ guaioxide ${ }^{[2]}$ ), furanose-derivatives (isosorbide ${ }^{[3]}, \operatorname{xylitan}^{[4]}$ ), lignans $s^{[5,6]}$ (asarinin, nectandrin B, burseran), macrolides (avermectin), fungal metabolites ${ }^{[7-10]}$ (aureonitol), and pharmaceutical drug leads (LY $379268^{[11]}$ and elenbecestat $\left.{ }^{[12]}\right)$.

Considering the relevance of tetrahydrofurans to drug discovery, a variety of strategies have been developed to access them in efficient ways. ${ }^{[13]}$ Synthesis strategies fall into one of two subcategories: (a) constructive synthesis, where the THF scaffold is assembled ${ }^{[14-23]}$ or (b) by an approach that functionalizes a preexisting THF core (e.g C-H functionalization, cross-coupling reactions ${ }^{[24-31]}$ The former category is of particular relevance to this report. We have been advancing the classic Cope rearrangement

Figure 1. Representative THF-containing natural products and pharmaceuticals
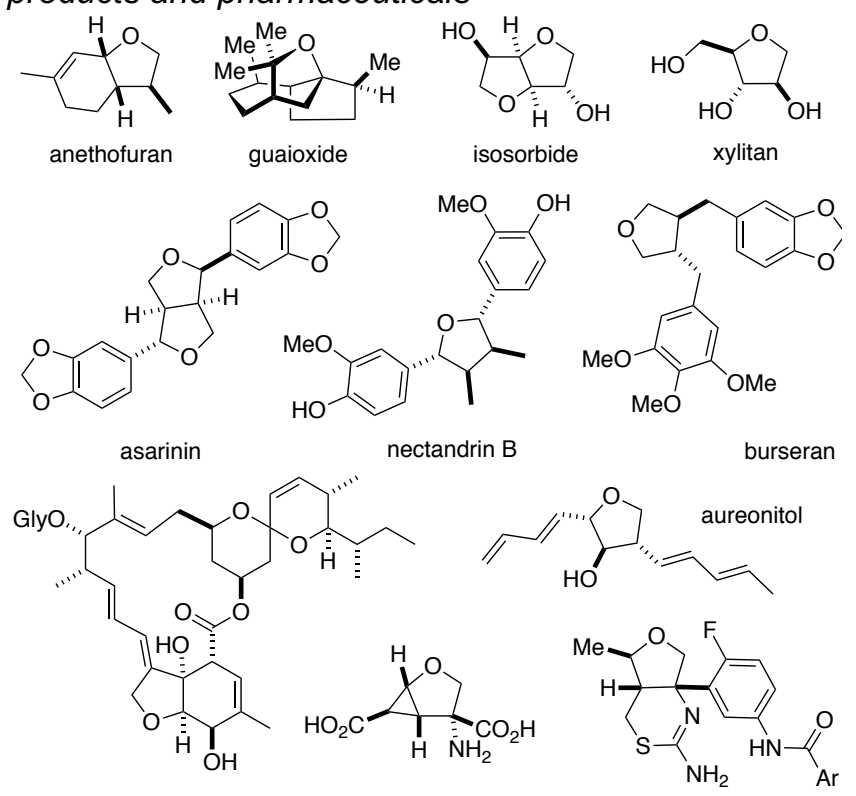

nectandrin B

burseran

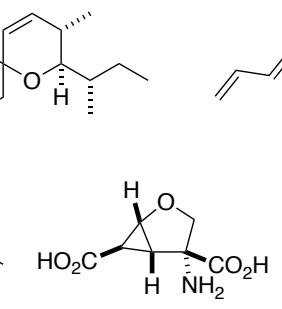

LY 379268

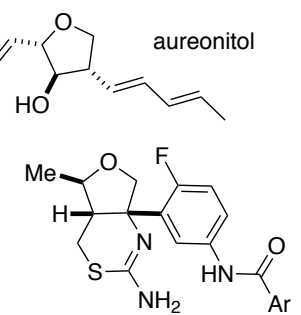

elenbecestat 
(that of 3,3-dicyano-1,5-dienes) for application in modern chemical synthesis. ${ }^{[32-37]}$ Considering that Cope rearrangement results in allylic transposition and an electrophilic alkylidenemalononitrile, we hypothesized that

Scheme 1. This proposal for THF synthesis and representative state of the art protocols.

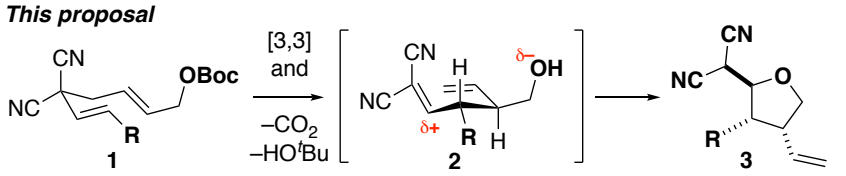

$\begin{array}{ccc}\text { simple 1,5- } & \text { allylic transposition and nucleophile/ } & \text { complex } \\ \text { electrophile generation } & \text { THFs }\end{array}$

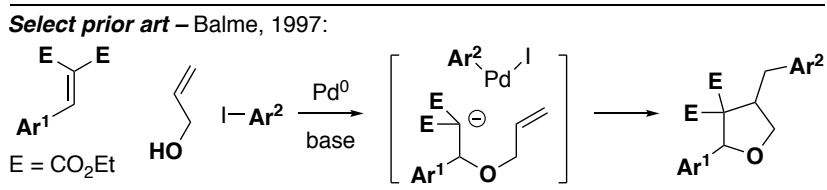

Select prior art-Yamamoto 1998 \& 2001 :

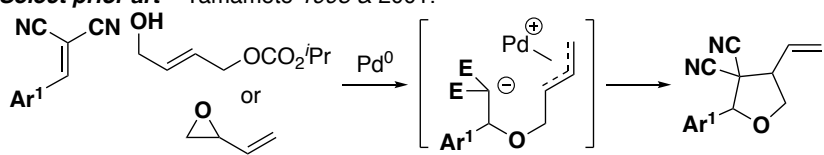

"trapping" with tethered nucleophiles could result in additional bond formation and structural complexity. Specifically, it was proposed that 1,5-dienes 1 derived from alkylidenemalononitrile and a cis-buten-1,4-diol derivative could undergo polythermal transformation involving Cope rearrangement, Boc-deprotection, and intramolecular oxy-Michael addition. Reviews of furan synthesis literature ${ }^{[13-31]}$ and "domino reactions ${ }^{[38,39] "}$ in general reveal that this proposal is unique and would

access novel trisubstituted THFs. The most relevant prior art is summarized in Scheme 1, which demonstrates the likelihood of oxy-Michael additions to alkylidenemalonic acid derivatives. Interestingly our proposal and these methods for comparison result in furans where the malonate/malononitrile functional group are at complementary locations about the THF core.

\section{Results and Discussion}

We began our studies by synthesizing diastereomeric 1,5-dienes E-1a and Z-1a from their corresponding

Scheme 2. Representative synthesis of either E- or Z1,5-dienes.
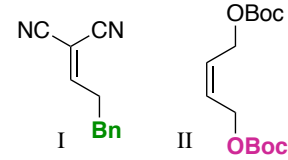

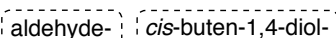
derived $: 1$, derived

${ }_{\mathrm{I}}^{\mathrm{NC}}$

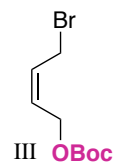

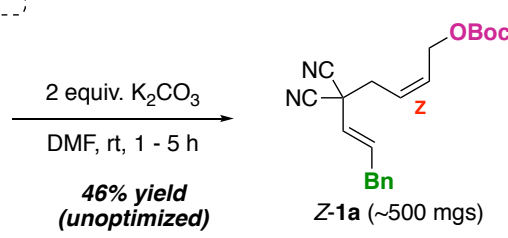

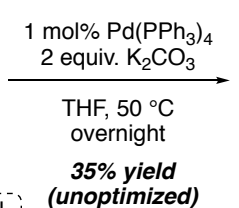

(unoptimized) aldehyde-derived Knoevenagel adduct and a cis-buten1,4-diol derivative (Scheme 2). Specifically, $E-1 a$ is prepared in $35 \%$ unoptimized yield via Tsuji-Trost reaction between alkylidenemalononitrile $\mathbf{I}$ and the bisboc-protected cis-buten-1,4-diol derivative II where Z-1a is derived from the allyl bromide III and prepared in $46 \%$ yield. Notably, via Pd-catalysis the cis-olefin on II is

isomerized to the trans-isomer during the deconjugative alkylation process. ${ }^{[40]}$ Finally, all 1,5 -dienes in this disclosure were prepared by this method in unoptimized yields ranging from $25-75 \%$ (See the supporting information for details). 
With E-1a and Z-1a in hand, we began examining the substrates for the desired thermal cascade to tetrahydrofurans. The desired products $3 \mathbf{a}$ and $\mathbf{3 b}$ could be formed when heated at $175{ }^{\circ} \mathrm{C}$ in toluene with good yield and diastereoselectivity (Scheme 3.). Interestingly, $\mathbf{3 a}$ and $\mathbf{3} \mathbf{b}$ were the only products detected in the crude mixture; none of the suspected intermediates such as [IntA], [Int-B], or their isomers were observed. The different diastereomers $E-1 \mathbf{a}$ and $Z-1 a$ react distinctly and diastereoselectively to yield their respective products. In both cases, the stereochemical outcomes can be
Scheme 3. Stereospecific furan synthesis via a thermal cascade.
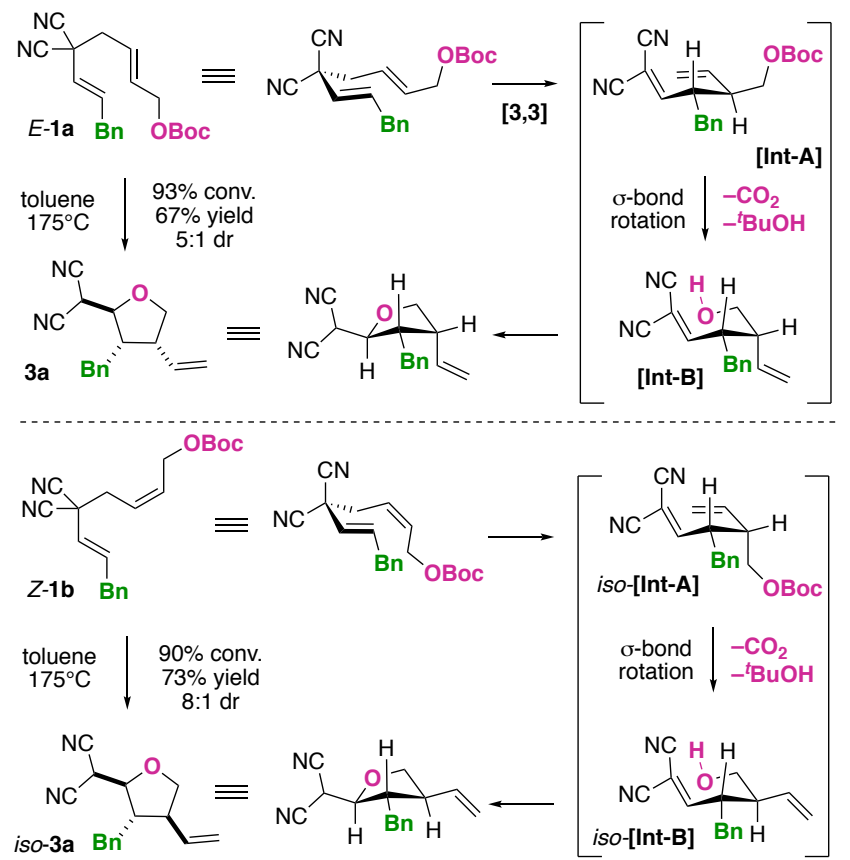
rationalized via a Zimmerman-Traxler model and an oxy-Michael addition yielding a pseudo-equatorial malononitrile functional group. Notably, the elevated temperatures in this transformation's current form are

Scheme 4. The Cope rearrangement and oxy-Michael addition are cooperative Tandem processes.
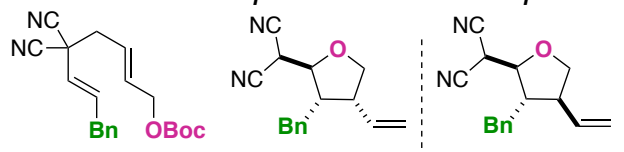

$\mathrm{CN}$

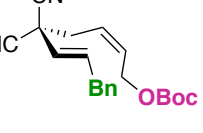
93\% conv. $90 \%$ conv
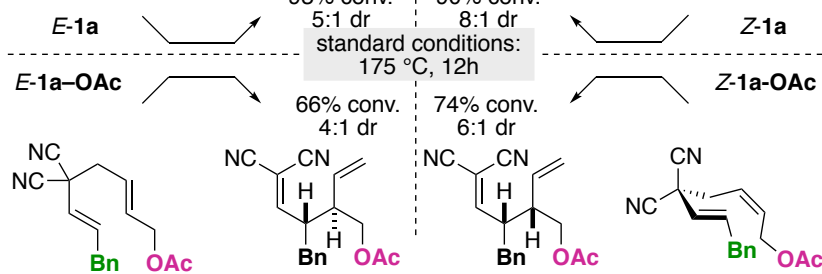

CC:

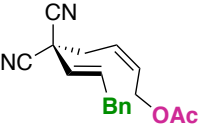

necessary: at $150{ }^{\circ} \mathrm{C}$ or lower in toluene, we observed diminished conversion of the 1,5-dienes. In this case, both Cope rearrangement and Boc-deprotection were sluggish over the same time period.

From our previous work on related 1,5-dienes, it was found that the classic Cope rearrangement often does not favor the product side of the equilibrium. ${ }^{[34,36]}$ Rather it was demonstrated that the Cope rearrangement could be promoted by a stoichiometric reductant that intermolecularly reacted with the product side of the equilibrium via chemoselective alkylidenemalononitrile reduction (the "reductive Cope rearrangement"). It was hypothesized that oxy-Michael addition was having a similar effect here. To examine this, we compared E-1a and Z-1a to substrates E-1a-OAc and Z-1a-OAc that have similar structure and electronics but cannot generate the nucleophilic hydroxyl group under the standard reaction conditions (Scheme 4). While E-1a transforms to the furan scaffolds with $93 \%$ conversion under standard conditions, E-1a-OAc had lower conversion (66\%). A similar trend was observed for the Z-1a and Z1a-OAc, though with slightly different ratios. This suggests that the tandem transformation is cooperative: The 
Cope equilibrium process will not reach full conversion on its own during the reaction time, rather is driven forward and rendered irreversible by the nucleophilic addition; the oxy-Michael reaction.

The scope of the furan synthesizing reaction was examined next (Scheme 5). It was found that the transformation was successful with a variety of substrates and could tolerate the incorporation of functional groups such as alkenes an ${ }^{-} d$ TBS-protected alcohols. Furthermore, the yield and diastereoselectivity remained consistent with the previously optimized yields. It should be noted that sterics about the 1,5-diene termini have an impact on the kinetics of the transformation. For example, 1e having an $\alpha$-isopropyl group reacted under the standard conditions to produce a mixture of the desired product $(50 \%$ yield, 9:1 dr) along with the Bocdeprotected substrate $\mathbf{4 e}$. However, you can re-subject the 1,5-diene-alcohol to the conditions and yield additional product. As a final note, this suggests that the order of events for the tandem reaction might be Bocdeprotection then Cope rearrangement.

Having an understanding of the scope of the transformation, we next examined chirality transfer from an existing stereocenter on the 1,5-diene for the cascade sequence. Unfortunately, in limited studies (only the one substrate disclosed herein has currently been examined to date), the existing stereocenter on 1f did not influence the diastereoselectivity of the Cope rearrangement (equation 1). Thus, two epimers (3f) were prepared via the

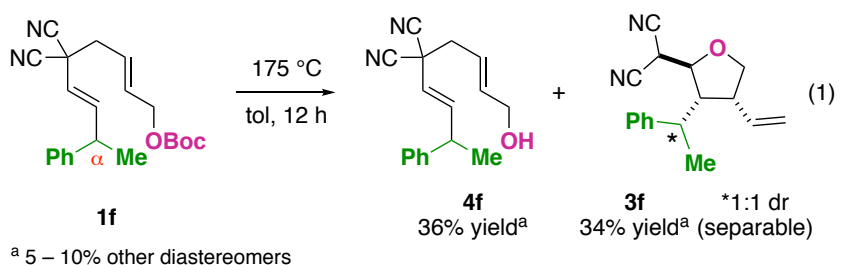
thermal cascade reaction. Notably, both epimers can be separated by silica gel chromatography and each individual epimer is $>20: 1 \mathrm{dr}$ from the tandem thermal transformation.

We next examined malonate-containing 1,5-dienes for tetrahydrofuran synthesis. Generally speaking, the malonate-1,5-dienes are less reactive to Cope rearrangement than malononitrile substrates. For example, 
Scheme 6. Reactivity of malonate-containing-1,5dienes.
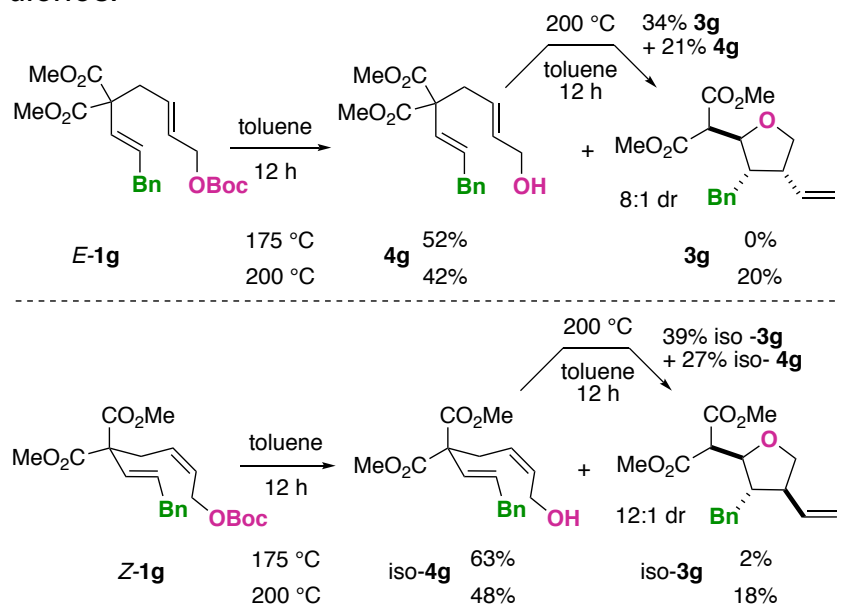

heating $E-1 \mathrm{~g}$ at $175{ }^{\circ} \mathrm{C}$ only produced the Bocdeprotected starting material $\mathbf{4 g}$ in $52 \%$ yield (Scheme 6 ). At $200{ }^{\circ} \mathrm{C}$, the desired product $\mathbf{3 g}$ was prepared in $20 \%$ yield as an $8: 1$ mixture of diastereomers along with $42 \%$ of the alcohol $\mathbf{4 g}$, which upon re-subjection to the standard conditions produced an additional $21 \%$ of the desired furan. Similar reactivity was observed with Z-1g.

As final results related to scope, we have identified a current limitation of this furan synthesis (equations 2). In

its current form, the transformation is limited to aldehyde- and butene-1,4-diol-derived 1,5-dienes, as outlined in the aforementioned schemes and equations. We encountered challenges when utilizing ketone-derived substrates (equation 2). Under the standard conditions, E-1h underwent Cope rearrangement (with significant
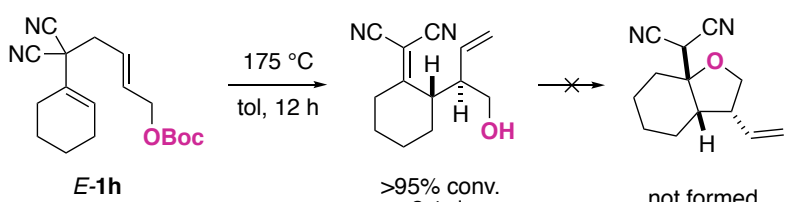

$>95 \%$ conv.
$2: 1 \mathrm{dr}$

not formed

Scheme 7. Representative functional group interconversion chemistry.

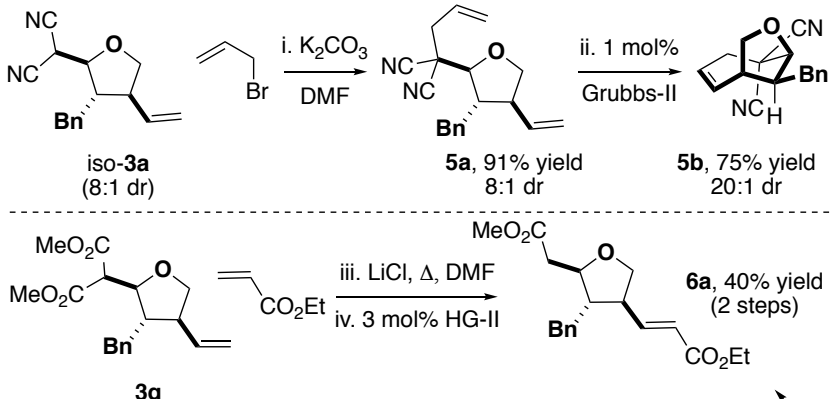

$$
\begin{aligned}
& 3 \mathrm{~g} \\
& (8: 1 \mathrm{dr})
\end{aligned}
$$

chain-length analogs )
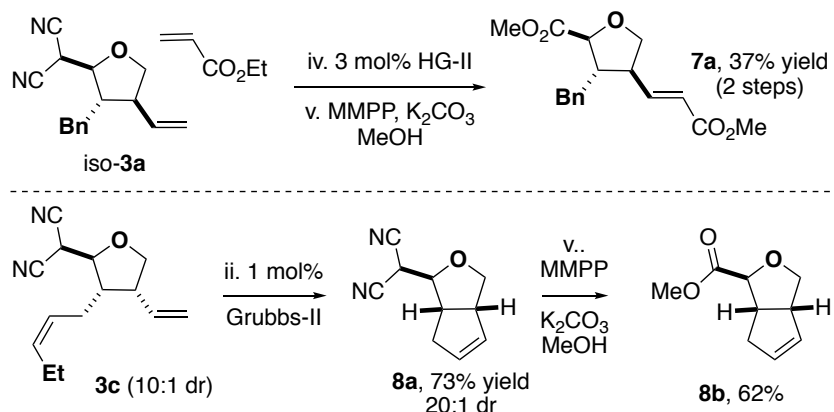

Conditions: i. 2 eq $\mathrm{K}_{2} \mathrm{CO}_{3}$, DMF, rt, 1 h. ii. 1 mol\% Grubbs II, toluene, $80^{\circ} \mathrm{C}, 12 \mathrm{~h}$ iii. 1 eq LiCl, 1 eq $\mathrm{H}_{2} \mathrm{O}$, DMF, $150^{\circ} \mathrm{C}$, 4 h. iv.) 3 mol\% Hoveyda-Grubbs II, 10 eq ethyl acrylate, toluene, $80^{\circ} \mathrm{C}, 12$ h. v.) 1 eq MMPP, 2 eq $\mathrm{K}_{2} \mathrm{CO}_{3}, \mathrm{MeOH}, \mathrm{rt}, 1 \mathrm{~h}$.

epimerization; 2:1 dr) and Boc deprotection but no furan product was detected. The potential reasons for the lack of oxy-Michael reactivity are many. For example, there could be steric or conformation issues with the nucleophilic attack. Alternatively, the reduced electrophilicity of the tetrasubstituted alkylidene may also play a role in the undesired outcome. In any case, more studies are needed to expand the reactivity to ketones.

For our final studies, we examined functional group interconversions on the THF scaffolds (Scheme 7). As a result of the reaction design, trisubstituted THFs with complementary and orthogonal functional groups are prepared. This allows for rapid structural diversification. For example, we prepared a bicyclic THF-containing architecture $\mathbf{5 b}$ via malononitrile allylation (to $\mathbf{5 a}$ ) followed by ring-closing metathesis ${ }^{[41]}$ in $68 \%$ yield over 2 steps. 
The malonate-containing THF scaffold can be converted to $6 a$ via Krapcho decarboxylation ${ }^{[42]}$ followed by crossmetathesis ${ }^{[43]}$ with ethyl acrylate in $40 \%$ yield over 2 steps. Interestingly, the analogous malononitrile scaffold iso-3a can be converted into a chain-length analog (6a vs $7 a)$ via oxidative decyanation ${ }^{[44-46]}$ then crossmetathesis. Finally, $\mathbf{3 c}$ was converted into the bicycloalkanes $\mathbf{8 b}$ via ring-closing metathesis (to $\mathbf{8 a}$ ) and oxidative decyanation.

In conclusion, we have developed a new methodology to synthesize trisubstituted and functionally dense tetrahydrofurans from readily available 1,5-dienes via a cooperative tandem process involving Cope rearrangement, OBoc-deprotection, and oxy-Michael addition. The transformation occurs with good yields and diastereoselectivities within the current scope. These discoveries set the stage for numerous follow-up studies related to increasing the scope and diversity of the transformation, developing asymmetric variants, and finding unique opportunities for the transformation in complex natural product and pharmaceutical molecule synthesis.

\section{Experimental Section}

Experimental procedures, compound characterization ( $\left.{ }^{1} \mathrm{NMR},{ }^{13} \mathrm{C} N \mathrm{NMR}, \mathrm{HRMS}\right)$, and spectral reprints are available in the Supporting Information.

\section{Acknowledgements}

This material is based upon work supported by the National Science Foundation under Grant No. 1844443. We thank the College of Liberal Arts and Sciences and the Department of Chemistry at the University of Florida for start-up funds. We thank the Mass Spectrometry Research and Education Center and their funding source: $\mathrm{NIH}$ S10 OD021758-01A1.

\section{References}

[1] G. Q. Zheng, P. M. Kenney, L. K. T. Lam, Planta Med. 1992, 58, 338-341.

[2] L. Tissandie, M. Gaysinski, H. Brevard, U. J. Meierhenrich, J.-J. Filippi, J. Nat. Prod. 2017, 80, $526-537$.

[3] M. Rose, R. Palkovits, ChemSusChem 2012, 5, 167-176.

[4] P. R. Elias, G. S. Coelho, V. F. Xavier, F. F. Hilario, J. G. Taylor, V. F. Xavier, J. P. A. Sales, A. J. Romanha, S. M. F. Murta, C. M. Carneiro, et al., Molecules 2016, 21, 1342-1355.

[5] J.-Y. Pan, S.-L. Chen, M.-H. Yang, J. Wu, J. Sinkkonen, K. Zou, Nat. Prod. Rep. 2009, 26, 1251-1292.

[6] X. Fang, X. Hu, Molecules 2018, 23, 3385-3407.

[7] F. Bohlmann, J. Ziesche, Phytochem. 1979, 18, 664-665.

[8] R. G. Marwah, M. O. Fatope, M. L. Deadman, Y. M. Al-Maqbali, J. Husband, Tetrahedron 2007, 63, 8174-8180.

[9] T. Nakazawa, K. Ishiuchi, M. Sato, Y. Tsunematsu, S. Sugimoto, Y. Gotanda, H. Noguchi, K. Hotta, K. Watanabe, J. Am. Chem. Soc. 2013, 135, 13446-13455.

[10] T. Asai, S. Morita, N. Shirata, T. Taniguchi, K. Monde, H. Sakurai, T. Ozeki, Y. Oshima, Org. Lett. 2012, 14, 5456-5459.

[11] J. A. Monn, M. J. Valli, S. M. Massey, M. M. Hansen, T. J. Kress, J. P. Wepsiec, A. R. Harkness, J. L. Grutsch Jr., R. A. Wright, B. G. Johnson, et al., J. Med. Chem. 1999, 42, 1027-1040. 
[12] J. Godyń, J. Jończyk, D. Panek, B. Malawska, Pharmacol. Reports 2016, 68, 127-138.

[13] J. P. Wolfe, M. B. Hay, Tetrahedron 2007, 63, 261-290.

[14] M. Gao, Y. Zhao, C. Zhong, S. Liu, P. Liu, Q. Yin, L. Hu, Org. Lett. 2019, 21, 5679-5684.

[15] P. Hoffmeyer, C. Schneider, European J. Org. Chem. 2019, 2019, 5326-5333.

[16] B. M. Trost, G. Mata, Angew. Chem. Int. Ed. 2018, 57, 12333-12337.

[17] S. Ali, H. Milanezi, T. M. F. Alves, C. F. Tormena, M. A. B. Ferreira, J. Org. Chem. 2018, 83, 76947713.

[18] J. Son, T. W. Reidl, K. H. Kim, D. J. Wink, L. L. Anderson, Angew. Chem. Int. Ed. 2018, 57, 6597-6600.

[19] Y. Xie, G.-J. Cheng, S. Lee, P. S. J. Kaib, W. Thiel, B. List, J. Am. Chem. Soc. 2016, 138, 1453814541.

[20] W. G. Shuler, L. A. Combee, I. D. Falk, M. K. Hilinski, European J. Org. Chem. 2016, 2016, 3335-3338.

[21] J. Sabbatani, N. Maulide, Angew. Chem. Int. Ed. 2016, 55, 6780-6783.

[22] X. Yuan, L. Lin, W. Chen, W. Wu, X. Liu, X. Feng, J. Org. Chem. 2016, 81, 1237-1243.

[23] S. M. Nicolle, W. Lewis, C. J. Hayes, C. J. Moody, Angew. Chem. Int. Ed. 2015, 54, 8485-8489.

[24] M. Shang, K. S. Feu, J. C. Vantourout, L. M. Barton, H. L. Osswald, N. Kato, K. Gagaring, C. W. McNamara, G. Chen, L. Hu, et al., Proc. Natl. Acad. Sci. 2019, 116, 8721 LP - 8727.

[25] G. Wang, X. Xin, Z. Wang, G. Lu, Y. Ma, L. Liu, Nat. Commun. 2019, 10, 1-9.

[26] Y. Yang, F. Yuan, X. Ren, G. Wang, W. Zhao, X. Tang, M. Guo, J. Org. Chem. 2019, 84, 4507-4516.

[27] P. Hoffmeyer, C. Schneider, J. Org. Chem. 2019, 84, 1079-1084.

[28] S. Lee, P. S. J. Kaib, B. List, J. Am. Chem. Soc. 2017, 139, 2156-2159.

[29] D. P. Affron, J. A. Bull, European J. Org. Chem. 2016, 2016, 139-149.

[30] J. Li, J. Zhang, H. Tan, D. Z. Wang, Org. Lett. 2015, 17, 2522-2525.

[31] D. Liu, C. Liu, H. Li, A. Lei, Angew. Chem. Int. Ed. 2013, 52, 4453-4456.

[32] O. Lahtigui, F. Emmetiere, W. Zhang, L. Jirmo, S. Toledo-Roy, J. C. Hershberger, J. M. Macho, A. J. Grenning, Angew. Chem. Int. Ed. 2016, 55, 15792-15796.

[33] S. K. Scott, A. J. Grenning, Angew. Chem. Int. Ed. 2017, 56, 8125-8129.

[34] E. Fereyduni, A. J. Grenning, Org. Lett. 2017, 19, 4130-4133.

[35] S. K. Scott, J. N. Sanders, K. E. White, R. A. Yu, K. N. Houk, A. J. Grenning, J. Am. Chem. Soc. 2018, 140, 16134-16139.

[36] P. Vertesaljai, R. Serrano, M. D. Mannchen, M. Williams, E. Semenova, A. J. Grenning, Org. Lett. 2019, 21, 5704-5707.

[37] E. Fereyduni, J. N. Sanders, G. Gonzalez, K. N. Houk, A. J. Grenning, Chem. Sci. 2018, 9, 8760-8764.

[38] H. Pellissier, Chem. Rev. 2013, 113, 442-524.

[39] L. F. Tietze, Chem. Rev. 1996, 96, 115-136.

[40] A. J. Blacker, M. L. Clarke, M. S. Loft, J. M. J. Williams, Org. Lett. 1999, 1, 1969-1971.

[41] M. E. Maier, Angew. Chem. Int. Ed. 2000, 39, 2073-2077.

[42] A. P. Krapcho, E. Ciganek, Org. React. (Hoboken, NJ, United States) 2013, 81, 1-535.

[43] R. H. Grubbs, S. J. Miller, G. C. Fu, Acc. Chem. Res. 1995, 28, 446-452.

[44] Y. Hayashi, J. Li, H. Asano, D. Sakamoto, European J. Org. Chem. 2019, 2019, 675-677.

[45] J. Li, M. J. Lear, Y. Hayashi, Angew. Chem. Int. Ed. 2016, 55, 9060-9064.

[46] S. Foester, O. Tverskoy, G. Helmchen, Synlett 2008, 2803-2806. 


\title{
Supporting Information
}

\section{Convergent synthesis of trisubstituted tetrahydrofurans via bis-thermally reactive 1,5-diene-tert-butyl carbonates.}

\author{
Fabien Emmetiere, Alexander J. Grenning
}

\section{Table of content}

1. General experimental details

2. General procedures

a. Knoevenagel adduct $\mathrm{S}_{\mathrm{N}} 2$ alkylation

i. Procedure A-1

ii. Procedure A-2

b. Knoevenagel adduct Tsuji-Trost alkylation

i. Procedure B-1

ii. Procedure B-2

c. Cope rearrangement/Oxy Michael addition cascade

i. Procedure $\mathrm{C}-1$

ii. Procedure $\mathrm{C}-2$

d. Specific experimental procedures

i. Allylation of iso-3a

ii. Ring-closing metathesis of $\mathbf{5 a} / \mathbf{5 b}$ and $\mathbf{3 c} / \mathbf{8 a}$

iii. Krapcho decarboxylation of $\mathbf{3 g}$

iv. Cross-metathesis of iso-3a and SI-6a

v. Oxidative esterification of SI-7a and 8a

vi. Retro-Oxy-Michael addition on 3a

3. ${ }^{1} \mathrm{H},{ }^{13} \mathrm{C}$ NMR \& mass spectrometry data

4. Structural and Stereochemical assignment control experiments

a. Conversion and diastereoselectivity ratio for Scheme 4

b. Stereochemical outcome of the Cope rearrangement (3 and 4 position)

c. Stereochemical outcome of the Cope rearrangement/Oxy-Michael (2 position)

5. ${ }^{1} \mathrm{H} \&{ }^{13} \mathrm{C}$ NMR spectral reprints

6. References

\section{General experimental details}

All commercial materials were used without further purification. Knoevenagel adducts were synthesized from modified reported literature procedures that are referenced along the way. All other synthetic protocols are outlined below. ${ }^{1} \mathrm{H}$ NMR and ${ }^{13} \mathrm{C}$ NMR spectra were recorded in $\mathrm{CDCl}_{3}$ using a $400 \mathrm{MHz}$ or $600 \mathrm{MHz}$ Varian VNMRS spectrometer (with $\mathrm{CHCl}_{3}$ residual peak as an internal standard) unless specified otherwise. All ${ }^{13} \mathrm{C}$ NMR spectra were recorded with complete proton decoupling. HRMS data were recorded on Agilent Time of Flight 6200 spectrometer. Reaction progress was monitored by thin-layer chromatography 
(TLC) and visualized by UV light, phosphomolybdic acid stain, and $\mathrm{KMnO}_{4}$ stain. Compounds were purified via silica gel column chromatography using Hexanes/Ethyl Acetate (Hex/EtOAc) solvent mixture.

All reactions were carried out using anhydrous solvents obtained dried by passing through activated alumina columns. Notation: Hex/EtOAc X \% reads "mixture of $10 \%$ ethyl acetate (EtOAc) in hexanes (Hex)".

\section{General procedures}

a. Knoevenagel adduct $\mathrm{S}_{\mathrm{N}} 2$ alkylation

i. Procedure A-1

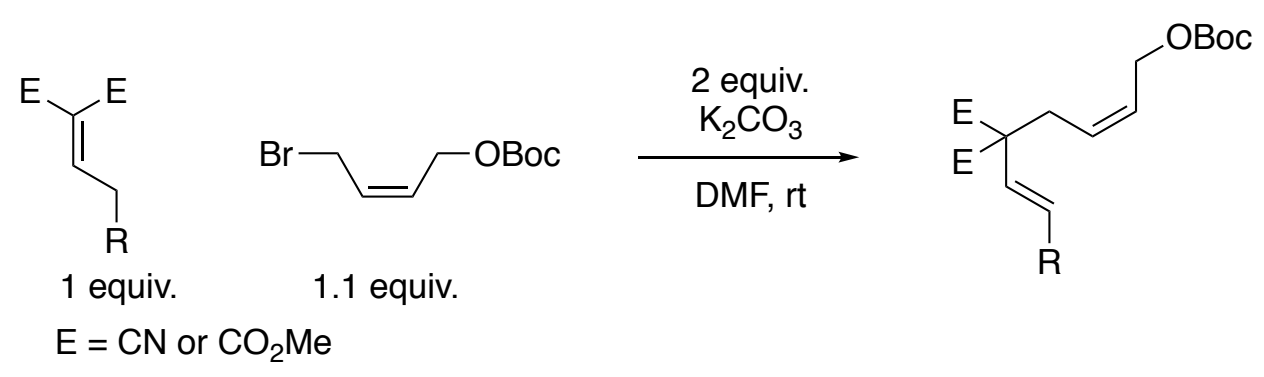

Pulverized $\mathrm{K}_{2} \mathrm{CO}_{3}$ (2 equiv.) was suspended in dry DMF in a flame-dried Schlenk flask under $\mathrm{N}_{2}$. A mixture of Knoevenagel adduct (1 equiv.) and (Z)-4-bromobut-2-en-1-yl tert-butyl carbonate [Bulletin of the Chemical Society of Japan, 92(5), 937-940; 2019] (1.1 equiv.) in DMF (resulting in 0.1 M concentration) was then added at room temperature. The reaction mixture was monitored by TLC. Once the reaction reached completion, the reaction mixture was quenched slowly using a $2 \mathrm{M} \mathrm{HCl}_{(\mathrm{aq})}$ solution until no more effervescence was observed. The resulting aqueous mixture was extracted with EtOAc three times. The organic layers were then combined and washed sequentially with $2 \mathrm{M} \mathrm{HCl}_{(\mathrm{aq})}$ and brine before being dried over $\mathrm{Na}_{2} \mathrm{SO}_{4}$. The excess solvent was removed under reduced pressure and the crude mixture was purified via silica gel column chromatography (Hex/EtOAc).

\section{ii. Procedure A-2}

Same procedure as above but with $\mathrm{NaH}$ (1 equiv.) as base.

b. Knoevenagel adduct Tsuji-Trost alkylation

i. Procedure B-1

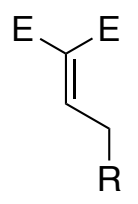

1 equiv. $\mathrm{E}=\mathrm{CN}$ or $\mathrm{CO}_{2} \mathrm{Me}$

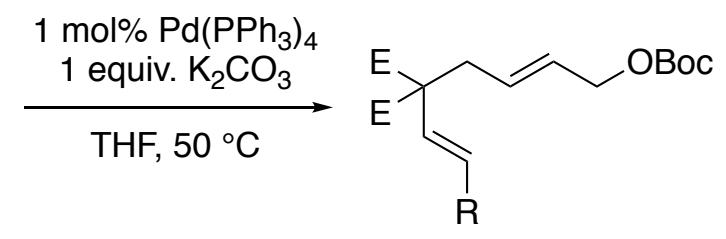


$\mathrm{Pd}\left(\mathrm{PPh}_{3}\right)_{4}(1 \mathrm{~mol} \%)$ and $\mathrm{K}_{2} \mathrm{CO}_{3}$ (1 equiv.) were charged in a flame-dried Schlenk flask under $\mathrm{N}_{2}$ and suspended in THF $(0.1 \mathrm{M})$. bis-Boc protected (Z)-but-2-ene-1,4-diol[Angewandte Chemie, International Edition, 49(48), 9270-9273, S9270/1-S9270/106; 2010] (1.1 equiv.) and Knoevenagel adduct (1 equiv.) were added sequentially in this order. The reaction mixture was heated at $50^{\circ} \mathrm{C}$ until completion (monitored by TLC). After completion, the reaction mixture was filtered through a short pad of silica eluted with EtOAc. The filtrate was concentrated down and the crude mixture was purified via silica column chromatography (Hex/EtOAc).

ii. Procedure B-2

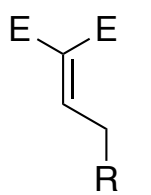

1 equiv.

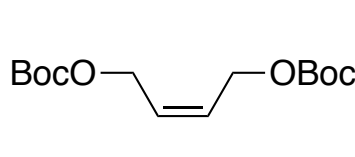

1.1 equiv.
$1 \mathrm{~mol} \% \mathrm{Pd}\left(\mathrm{PPh}_{3}\right)_{4}$

1 equiv. $\mathrm{NaH}$

DMF, rt

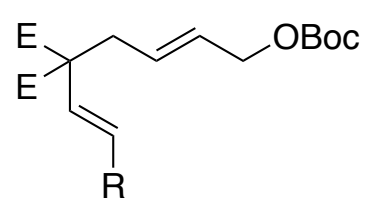

$$
\mathrm{E}=\mathrm{CN} \text { or } \mathrm{CO}_{2} \mathrm{Me}
$$

$\mathrm{Pd}\left(\mathrm{PPh}_{3}\right)_{4}(1 \mathrm{~mol} \%)$ and $\mathrm{NaH}$ (1 equiv.) were charged in a flame-dried Schlenk flask under $\mathrm{N}_{2}$ and suspended in DMF (0.1 M). bis-Boc protected (Z)-but-2-ene-1,4-diol [Angewandte Chemie, International Edition, 49(48), 9270-9273, S9270/1-S9270/106; 2010] (1.1 equiv.) and Knoevenagel adduct (1 equiv.) were added sequentially in this order. The reaction mixture was stirred at room temperature until completion (monitored by TLC). Once the reaction reached completion, the reaction mixture was quenched slowly using a $2 \mathrm{M} \mathrm{HCl}_{(\mathrm{aq})}$ solution until no more effervescence was observed. The resulting aqueous mixture was extracted with EtOAc three times. The organic layers were then combined and washed sequentially with $2 \mathrm{M} \mathrm{HCl}_{(\mathrm{aq})}$ and brine before being dried over $\mathrm{Na}_{2} \mathrm{SO}_{4}$. The excess solvent was removed under reduced pressure and the crude mixture was purified via silica gel column chromatography (Hex/EtOAc).

\section{c. Cope rearrangement/Oxy Michael addition cascade}

\section{i. Procedure $\mathrm{C}-1$}<smiles>[R]C=CC(F)(F)C/C=C\COC(C)(C)C</smiles>

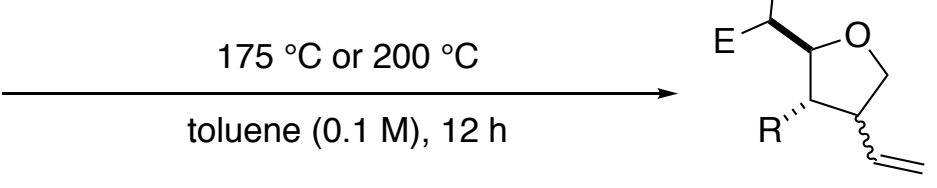

1 equiv.

$$
\mathrm{E}=\mathrm{CN} \text { or } \mathrm{CO}_{2} \mathrm{Me}
$$

Alkylated Knoevenagel precursor was charged in a flame-dried microwave pressured flask under $\mathrm{N}_{2}$. Toluene is added $(0.1 \mathrm{M})$ and the pressured flask was sealed. The reaction mixture was heated at $175^{\circ} \mathrm{C}$ for $12 \mathrm{~h}$. After $12 \mathrm{~h}$ reaction, the flask was cooled down to room temperature and toluene was removed under reduced pressure. The resulting crude mixture was purified via silica gel column chromatography (Hex/EtOAc).

\section{ii. Procedure $\mathrm{C}-2$}

Same as Procedure C-1 but at $200{ }^{\circ} \mathrm{C}$. 


\section{d. Specific experimental procedures}

i. Allylation of iso-3a and 3a
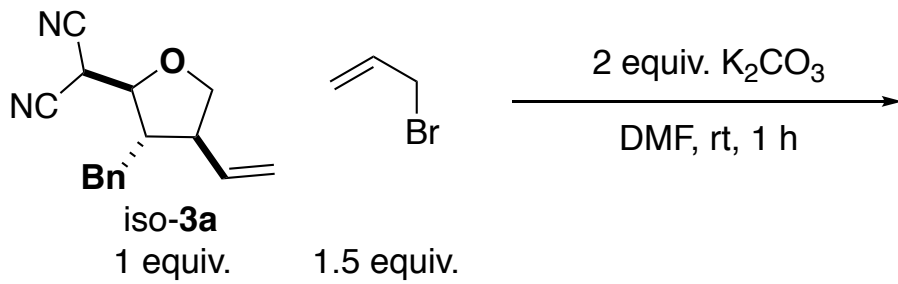

1.5 equiv.

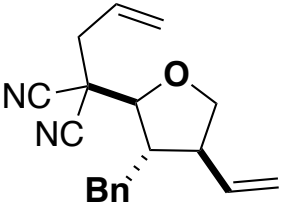

$5 a, 91 \%$

$\mathrm{K}_{2} \mathrm{CO}_{3}$ (2 equiv., $22 \mathrm{mg}, 0.16 \mathrm{mmol}$ ) was charged in a flame-dried Schlenk flask under $\mathrm{N}_{2}$ and suspended in DMF $(0.5 \mathrm{~mL})$. iso-3a (1 equiv., $20 \mathrm{mg}, 0.079 \mathrm{mmol}$ ) in $0.5 \mathrm{~mL}$ DMF was then added immediately followed by allyl bromide ( 1.5 equiv., $10 \mu \mathrm{L}, 0.12 \mathrm{mmol}$ ) at room temperature. The reaction was stirred for $1 \mathrm{~h}$ at room temperature and was then quenched slowly using a $2 \mathrm{M} \mathrm{HCl}_{(\mathrm{aq})}(3 \mathrm{~mL})$ solution until no more effervescence was observed. The resulting aqueous mixture was extracted with EtOAc three times $(3 \times 10$ $\mathrm{mL})$. The organic layers were then combined and washed sequentially with $2 \mathrm{M} \mathrm{HCl}_{(\mathrm{aq})}(10 \mathrm{~mL})$ and brine $(10 \mathrm{~mL})$ before being dried over $\mathrm{Na}_{2} \mathrm{SO}_{4}$. The excess solvent was removed under reduced pressure and $\mathbf{5 a}$ was sufficiently pure to be used in the next step without further purification $(21 \mathrm{mg}, 91 \%$ yield, colorless oil, 8:1 d.r., major reported below).

${ }^{1}$ H NMR (400 MHz, Chloroform- $d$ ) $\delta 7.35-7.28$ (m, 2H), 7.25 - 7.17 (m, 3H), 5.91 (ddt, $J=17.3,10.4$, $7.3 \mathrm{~Hz}, 1 \mathrm{H}), 5.60(\mathrm{dt}, J=17.9,9.2 \mathrm{~Hz}, 1 \mathrm{H}), 5.46-5.36(\mathrm{~m}, 2 \mathrm{H}), 5.05(\mathrm{~d}, J=6.1 \mathrm{~Hz}, 1 \mathrm{H}), 5.02(\mathrm{~s}, 1 \mathrm{H})$, $3.94(\mathrm{dd}, J=7.6,3.2 \mathrm{~Hz}, 2 \mathrm{H}), 3.82(\mathrm{t}, J=9.2 \mathrm{~Hz}, 1 \mathrm{H}), 3.11(\mathrm{dd}, J=14.0,4.6 \mathrm{~Hz}, 1 \mathrm{H}), 2.93(\mathrm{dd}, J=14.1$, $7.3 \mathrm{~Hz}, 1 \mathrm{H}), 2.86-2.75(\mathrm{~m}, 2 \mathrm{H}), 2.66-2.49(\mathrm{~m}, 2 \mathrm{H})$.

${ }^{13}$ C NMR $\left(101 \mathrm{MHz}, \mathrm{CDCl}_{3}\right) \delta 137.28,135.42,130.14,128.67,127.00,123.36,118.26,114.58,114.11$, 83.57, 73.32, 50.29, 49.57, 42.91, 39.64, 37.31.
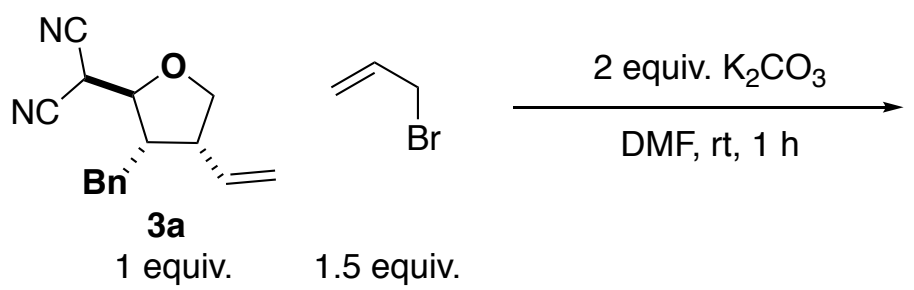

1.5 equiv.

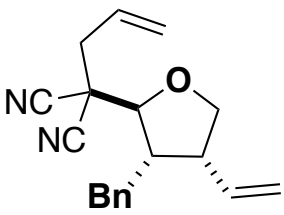

iso-5a, quant.

$\mathrm{K}_{2} \mathrm{CO}_{3}$ (2 equiv., $22 \mathrm{mg}, 0.16 \mathrm{mmol}$ ) was charged in a flame-dried Schlenk flask under $\mathrm{N}_{2}$ and suspended in DMF (0.5 mL). 3a (1 equiv., $19 \mathrm{mg}, 0.078 \mathrm{mmol}$ ) in $0.5 \mathrm{~mL}$ DMF was then added immediately followed by allyl bromide ( 1.5 equiv., $10 \mu \mathrm{L}, 0.12 \mathrm{mmol}$ ) at room temperature. The reaction was stirred for $1 \mathrm{~h}$ at room temperature and was then quenched slowly using a $2 \mathrm{M} \mathrm{HCl}_{(\mathrm{aq})}(3 \mathrm{~mL})$ solution until no more effervescence was observed. The resulting aqueous mixture was extracted with EtOAc three times $(3 \times 10$ $\mathrm{mL})$. The organic layers were then combined and washed sequentially with $2 \mathrm{M} \mathrm{HCl}_{(\mathrm{aq})}(10 \mathrm{~mL})$ and brine $(10 \mathrm{~mL})$ before being dried over $\mathrm{Na}_{2} \mathrm{SO}_{4}$. The excess solvent was removed under reduced pressure and $\mathbf{5 a}$ was sufficiently pure to be used in the next step without further purification ( $22 \mathrm{mg}$, quantitative yield, colorless oil, 5:1 d.r., major reported below).

${ }^{1}$ H NMR $(600 \mathrm{MHz}$, Chloroform- $d$ ) $\delta 7.32(\mathrm{t}, J=7.5 \mathrm{~Hz}, 2 \mathrm{H}), 7.26-7.20(\mathrm{~m}, 3 \mathrm{H}), 5.80$ (tq, $J=16.7,8.4$, $7.3 \mathrm{~Hz}, 2 \mathrm{H}), 5.37(\mathrm{~d}, J=10.2 \mathrm{~Hz}, 1 \mathrm{H}), 5.33(\mathrm{~d}, J=17.0 \mathrm{~Hz}, 1 \mathrm{H}), 5.26(\mathrm{~d}, J=10.3 \mathrm{~Hz}, 1 \mathrm{H}), 5.16(\mathrm{~d}, J=$ $17.2 \mathrm{~Hz}, 1 \mathrm{H}), 4.19(\mathrm{dd}, J=8.6,5.9 \mathrm{~Hz}, 1 \mathrm{H}), 3.96(\mathrm{~d}, J=4.6 \mathrm{~Hz}, 1 \mathrm{H}), 3.86(\mathrm{dd}, J=8.6,6.1 \mathrm{~Hz}, 1 \mathrm{H}), 3.21$ $(\mathrm{p}, J=6.7 \mathrm{~Hz}, 1 \mathrm{H}), 2.93-2.87(\mathrm{~m}, 1 \mathrm{H}), 2.84-2.77(\mathrm{~m}, 2 \mathrm{H}), 2.58(\mathrm{dd}, J=14.0,7.0 \mathrm{~Hz}, 1 \mathrm{H}), 2.45(\mathrm{dd}, J=$ $13.9,7.6 \mathrm{~Hz}, 1 \mathrm{H})$. 
${ }^{13}$ C NMR $\left(151 \mathrm{MHz}, \mathrm{CDCl}_{3}\right) \delta 138.65,133.97,129.27,128.87,128.63,126.90,123.18,119.02,114.48$, $113.81,83.70,73.47,48.58,46.32,43.17,39.37,34.79$.

ii. Ring-closing metathesis of $\mathbf{5 a} / \mathbf{5 b}$ and $\mathbf{3 c} / \mathbf{8 a}$
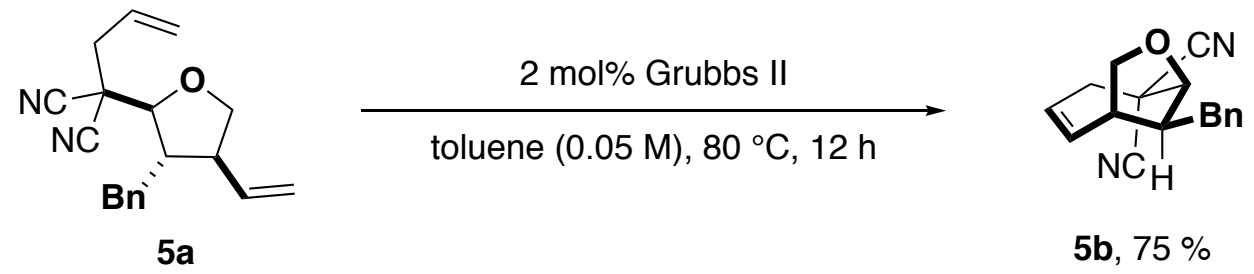

1 equiv.

Grubbs $2^{\text {nd }}$ generation catalyst $(2 \mathrm{~mol} \%, 1 \mathrm{mg}, 0.0014 \mathrm{mmol}$ ) was charged in a flame-dried Schlenk flask under $\mathrm{N}_{2}$ and dissolved in toluene $(0.4 \mathrm{~mL})$. 5a (1 equiv., $\left.20 \mathrm{mg}, 0.068 \mathrm{mmol}\right)$ in $1 \mathrm{~mL}$ toluene $(0.05 \mathrm{M}$ final concentration) was added at room temperature. The reaction mixture was then stirred at $80{ }^{\circ} \mathrm{C}$ for 12 h. After $12 \mathrm{~h}$, the reaction mixture was cooled down to room temperature and the solvent was removed under reduced pressure. The crude residue was purified via silica gel column chromatography (Hex/EtOAc $10 \%$ ) yielding bicyclic $5 \mathbf{b}$ as a colorless oil (13.5 mg, $75 \%$, 8:1 d.r., major reported below).

${ }^{1}$ H NMR $(600 \mathrm{MHz}$, Chloroform- $d$ ) $\delta 7.35(\mathrm{t}, J=7.6 \mathrm{~Hz}, 2 \mathrm{H}), 7.30-7.26(\mathrm{~m}, 1 \mathrm{H}), 7.20(\mathrm{~d}, J=7.3 \mathrm{~Hz}$, $2 \mathrm{H}), 6.28(\mathrm{ddd}, J=11.5,8.7,3.1 \mathrm{~Hz}, 1 \mathrm{H}), 5.64(\mathrm{ddd}, J=11.7,8.6,3.5 \mathrm{~Hz}, 1 \mathrm{H}), 4.63(\mathrm{~s}, 1 \mathrm{H}), 4.39(\mathrm{dd}, J=$ 8.7, $6.6 \mathrm{~Hz}, 1 \mathrm{H}), 4.17(\mathrm{dd}, J=8.7,1.3 \mathrm{~Hz}, 1 \mathrm{H}), 3.10(\mathrm{dt}, J=15.9,3.3 \mathrm{~Hz}, 1 \mathrm{H}), 2.79$ (q, $J=9.0,8.5 \mathrm{~Hz}$, $1 \mathrm{H}), 2.74(\mathrm{q}, J=5.9 \mathrm{~Hz}, 2 \mathrm{H}), 2.71(\mathrm{~d}, J=5.9 \mathrm{~Hz}, 1 \mathrm{H}), 2.70-2.66(\mathrm{~m}, 1 \mathrm{H})$.

${ }^{13}$ C NMR (151 MHz, $\left.\mathrm{CDCl}_{3}\right) \delta 140.49,137.97,129.07,129.04,127.16,122.70,115.96,114.38,85.72$, 75.42, 50.04, 41.13, 38.94, 38.15, 31.22.

HRMS (ESI - TOF) m/z: Calcd for $\mathrm{C}_{17} \mathrm{H}_{15} \mathrm{~N}_{2} \mathrm{O}[\mathrm{M}-\mathrm{H}]^{-} 263.1179$, found 263.1183.

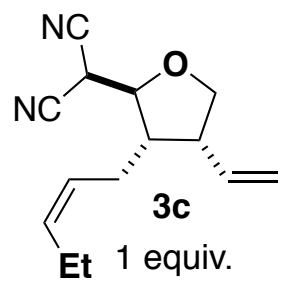

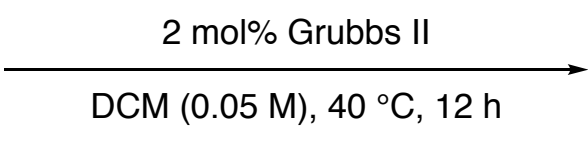

Et 1 equiv.

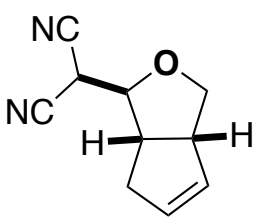

$8 a, 73 \%$

Grubbs $2^{\text {nd }}$ generation catalyst $(2 \mathrm{~mol} \%, 1.5 \mathrm{mg}, 0.0017 \mathrm{mmol})$ was charged in a flame-dried Schlenk flask under $\mathrm{N}_{2}$ and dissolved in DCM $(0.7 \mathrm{~mL})$. 3c (1 equiv., $\left.20 \mathrm{mg}, 0.086 \mathrm{mmol}\right)$ in $1 \mathrm{~mL} \mathrm{DCM}(0.05 \mathrm{M}$ final concentration) was added at room temperature. The reaction mixture was then stirred at $40{ }^{\circ} \mathrm{C}$ for $12 \mathrm{~h}$. After $12 \mathrm{~h}$, the reaction mixture was cooled down to room temperature and the solvent was removed under reduced pressure. The crude residue was purified via silica gel column chromatography (Pentanes/ $\mathrm{Et}_{2} \mathrm{O} 20$ \%) yielding bicyclic 8a as a colorless oil (11 mg, $73 \%$, 7:1 d.r., major reported below).

${ }^{1}$ H NMR $(600 \mathrm{MHz}$, Chloroform-d) $\delta 5.74(\mathrm{dq}, J=4.7,2.2 \mathrm{~Hz}, 1 \mathrm{H}), 5.66(\mathrm{dq}, J=5.7,2.1 \mathrm{~Hz}, 1 \mathrm{H}), 4.28$ $(\mathrm{dd}, J=8.9,7.3 \mathrm{~Hz}, 1 \mathrm{H}), 3.96(\mathrm{dd}, J=6.3,5.2 \mathrm{~Hz}, 1 \mathrm{H}), 3.92(\mathrm{~d}, J=5.2 \mathrm{~Hz}, 1 \mathrm{H}), 3.83$ (dd, $J=8.9,3.6 \mathrm{~Hz}$, $1 \mathrm{H}), 3.63$ (dddq, $J=9.8,5.9,3.9,2.2 \mathrm{~Hz}, 1 \mathrm{H}), 2.91$ (tdd, $J=8.0,6.3,1.6 \mathrm{~Hz}, 1 \mathrm{H}), 2.75$ (ddq, $J=17.2,7.8$, $2.4 \mathrm{~Hz}, 1 \mathrm{H}), 2.40(\mathrm{dp}, J=17.2,2.1 \mathrm{~Hz}, 1 \mathrm{H})$.

${ }^{13}$ C NMR $\left(151 \mathrm{MHz}, \mathrm{CDCl}_{3}\right) \delta 132.20,130.35,111.30,110.97,84.25,74.33,52.60,46.43,37.25,28.33$. HRMS (ESI - TOF) m/z: Calcd for $\mathrm{C}_{10} \mathrm{H}_{9} \mathrm{~N}_{2} \mathrm{O}[\mathrm{M}-\mathrm{H}]^{-}$173.0720, found 173.0715. 

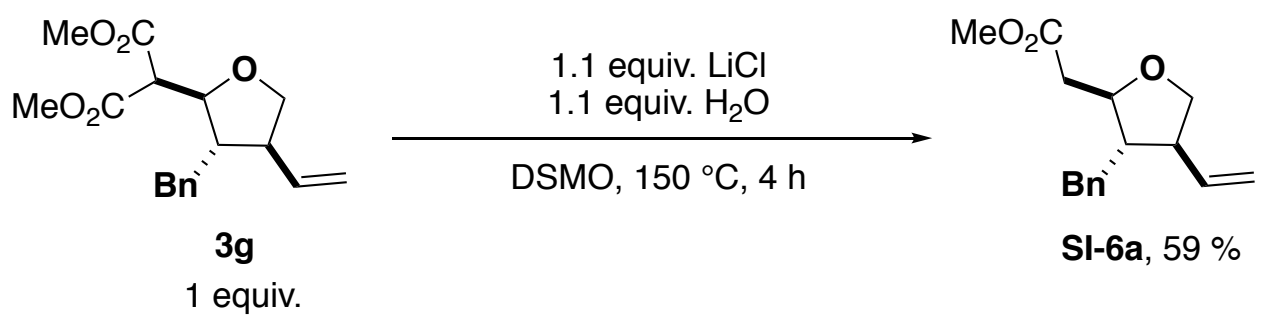

$3 \mathbf{g}$ (1 equiv., $35 \mathrm{mg}, 0.11 \mathrm{mmol}$ ) was dissolved in $0.5 \mathrm{~mL}$ DMSO is a microwave pressured flask. LiCl (1.1 equiv. $5 \mathrm{mg}, 0.12 \mathrm{mmol})$ and $\mathrm{H}_{2} \mathrm{O}(\sim 1.1$ equiv., $7 \mu \mathrm{L}, \sim 0.12 \mathrm{mmol})$ were added and the flask was sealed. The reaction mixture was heated at $150{ }^{\circ} \mathrm{C}$ for $4 \mathrm{~h}$. After completion, the reaction mixture was cooled down to room temperature and diluted with a $2 \mathrm{M} \mathrm{HCl}_{(\mathrm{aq})}(5 \mathrm{~mL})$ solution. The resulting aqueous mixture was extracted with EtOAc three times $(3 \times 5 \mathrm{~mL})$. The organic layers were then combined and washed sequentially with $2 \mathrm{M} \mathrm{HCl}_{(\mathrm{aq})}(10 \mathrm{~mL})$ and brine $(10 \mathrm{~mL})$ before being dried over $\mathrm{Na}_{2} \mathrm{SO}_{4}$. The excess solvent was removed under reduced pressure and the crude residue was purified via silica gel column chromatography (Hex/EtOAc $10 \%$ ). SI-6a was obtained as a colorless oil (17 mg, 59 \%, 8:1 d.r., major reported below).

${ }^{1}$ H NMR (400 MHz, Chloroform- $d$ ) $\delta 7.31-7.26(\mathrm{~m}, 3 \mathrm{H}), 7.23-7.18(\mathrm{~m}, 1 \mathrm{H}), 7.18-7.13(\mathrm{~m}, 2 \mathrm{H}), 5.72$ $-5.59(\mathrm{~m}, 1 \mathrm{H}), 5.15-5.00(\mathrm{~m}, 2 \mathrm{H}), 4.17-4.05(\mathrm{~m}, 1 \mathrm{H}), 4.01-3.91(\mathrm{~m}, 1 \mathrm{H}), 3.70-3.57(\mathrm{~m}, 3 \mathrm{H}), 2.94$ $(\mathrm{dt}, J=13.6,5.5 \mathrm{~Hz}, 1 \mathrm{H}), 2.66-2.52(\mathrm{~m}, 2 \mathrm{H}), 2.22(\mathrm{dd}, J=15.3,8.9 \mathrm{~Hz}, 1 \mathrm{H}), 2.11-2.06(\mathrm{~m}, 1 \mathrm{H}), 1.94$ (qt, $J=8.9,3.7 \mathrm{~Hz}, 1 \mathrm{H}$ ).

${ }^{13}$ C NMR $\left(101 \mathrm{MHz}, \mathrm{CDCl}_{3}\right) \delta 171.79,139.42,137.61,129.15,129.13,128.63,126.52,116.99,80.89$, 71.75, 51.96, 51.79, 50.98, 40.03, 37.40.

HRMS (ESI - TOF) m/z: Calcd for $\mathrm{C}_{16} \mathrm{H}_{20} \mathrm{NaO}_{3}[\mathrm{M}+\mathrm{Na}]^{+} 283.1305$, found 283.1293.

\section{iv. Cross-metathesis of iso-3a and SI-6a}
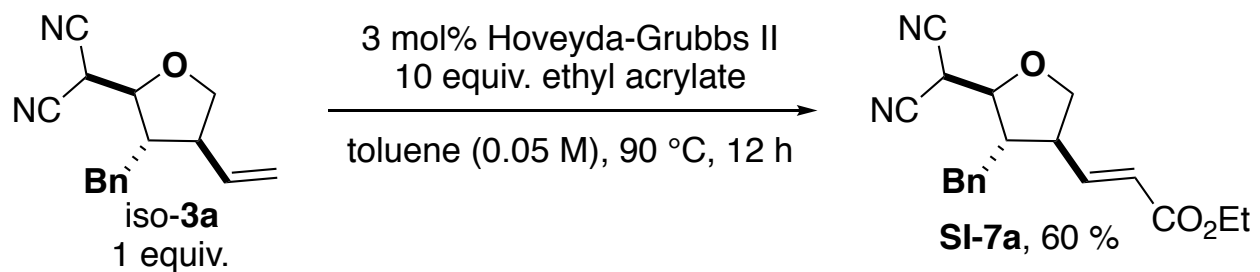

Hoveyda-Grubbs $2^{\text {nd }}$ generation catalyst $(3 \mathrm{~mol} \%, 1.5 \mathrm{mg}, 0.0024 \mathrm{mmol}$ ) was charged in a flame-dried Schlenk flaks under $\mathrm{N}_{2}$ and dissolved in $1 \mathrm{~mL}$ toluene. iso-3a (1 equiv., $20 \mathrm{mg}, 0.08 \mathrm{mmol}$ ) was added in $0.5 \mathrm{~mL}$ toluene $(0.05 \mathrm{M}$ final concentration) followed by ethyl acrylate (10 equiv., $84 \mu \mathrm{L}, 0.8 \mathrm{mmol})$. The reaction flask was sealed and heated at $90^{\circ} \mathrm{C}$ for $12 \mathrm{~h}$. After $12 \mathrm{~h}$, the reaction mixture was cooled down to room temperature and the solvent was removed under reduced pressure. The crude residue was purified via silica gel column chromatography (Hex/EtOAc $20 \%$ ) yielding SI-7a as a yellowish oil (15 mg, 60 \%, 8:1 d.r., major reported below).

${ }^{1}$ H NMR $(600 \mathrm{MHz}$, Chloroform- $d) \delta 7.37(\mathrm{t}, J=7.5 \mathrm{~Hz}, 2 \mathrm{H}), 7.32-7.28(\mathrm{~m}, 1 \mathrm{H}), 7.17(\mathrm{~d}, J=6.9 \mathrm{~Hz}$, 2H), 6.79 (dd, $J=15.6,9.0 \mathrm{~Hz}, 1 \mathrm{H}), 5.94(\mathrm{~d}, J=15.5 \mathrm{~Hz}, 1 \mathrm{H}), 4.22$ (q, $J=7.2 \mathrm{~Hz}, 2 \mathrm{H}), 4.14(\mathrm{dd}, J=8.9$, $7.7 \mathrm{~Hz}, 1 \mathrm{H}), 4.09(\mathrm{dd}, J=8.5,2.7 \mathrm{~Hz}, 1 \mathrm{H}), 3.91(\mathrm{dd}, J=9.9,8.9 \mathrm{~Hz}, 1 \mathrm{H}), 3.12(\mathrm{dd}, J=13.7,4.9 \mathrm{~Hz}, 1 \mathrm{H})$, $2.96-2.89(\mathrm{~m}, 1 \mathrm{H}), 2.73(\mathrm{~d}, J=2.6 \mathrm{~Hz}, 1 \mathrm{H}), 2.57(\mathrm{dd}, J=13.7,10.1 \mathrm{~Hz}, 1 \mathrm{H}), 2.41(\mathrm{tdd}, J=10.0,8.5,5.0$ $\mathrm{Hz}, 1 \mathrm{H}), 1.31(\mathrm{t}, J=7.1 \mathrm{~Hz}, 3 \mathrm{H})$. 
${ }^{13}$ C NMR $\left(151 \mathrm{MHz}, \mathrm{CDCl}_{3}\right) \delta 165.65,143.60,137.45,129.64,128.64,127.91,125.17,111.35,110.75$, 82.00, 72.87, 60.89, 50.36, 49.29, 36.94, 28.61, 14.37 .

HRMS (ESI - TOF) m/z: Calcd for $\mathrm{C}_{19} \mathrm{H}_{20} \mathrm{~N}_{2} \mathrm{NaO}_{3}[\mathrm{M}+\mathrm{Na}]^{+}$347.1366, found 347.1375.
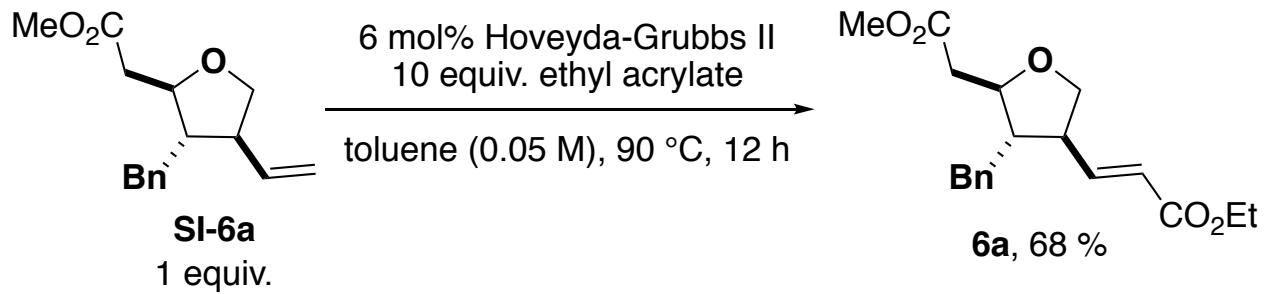

Hoveyda-Grubbs $2^{\text {nd }}$ generation catalyst $(6 \mathrm{~mol} \%, 2 \mathrm{mg}, 0.0029 \mathrm{mmol})$ was charged in a flame-dried Schlenk flaks under $\mathrm{N}_{2}$ and dissolved in $0.5 \mathrm{~mL}$ toluene. SI-a (1 equiv., $15 \mathrm{mg}, 0.057 \mathrm{mmol}$ ) was added in $0.5 \mathrm{~mL}$ toluene $(0.05 \mathrm{M}$ final concentration) followed by ethyl acrylate (10 equiv., $80 \mu \mathrm{L}, 0.57 \mathrm{mmol})$. The reaction flask was sealed and heated at $90{ }^{\circ} \mathrm{C}$ for $12 \mathrm{~h}$. After $12 \mathrm{~h}$, the reaction mixture was cooled down to room temperature and the solvent was removed under reduced pressure. The crude residue was purified via silica gel column chromatography (Hex/EtOAc $15 \%$ ) yielding 6a as a yellow oil (13.5 mg, 68 \%, 6:1 d.r., major reported below).

${ }^{1}$ H NMR (400 MHz, Chloroform- $d$ ) $\delta 7.30-7.27(\mathrm{~m}, 2 \mathrm{H}), 7.22-7.16(\mathrm{~m}, 1 \mathrm{H}), 7.16-7.11(\mathrm{~m}, 2 \mathrm{H}), 6.72$ $(\mathrm{dd}, J=15.6,9.2 \mathrm{~Hz}, 1 \mathrm{H}), 5.73(\mathrm{dd}, J=15.6,0.9 \mathrm{~Hz}, 1 \mathrm{H}), 4.17(\mathrm{q}, J=7.1 \mathrm{~Hz}, 2 \mathrm{H}), 4.14-4.07(\mathrm{~m}, 1 \mathrm{H})$, $3.96(\mathrm{dd}, J=8.9,7.6 \mathrm{~Hz}, 1 \mathrm{H}), 3.70-3.65(\mathrm{~m}, 2 \mathrm{H}), 3.65(\mathrm{~s}, 3 \mathrm{H}), 2.85(\mathrm{dd}, J=13.7,6.1 \mathrm{~Hz}, 1 \mathrm{H}), 2.76(\mathrm{tt}, J$ $=8.6,0.9 \mathrm{~Hz}, 1 \mathrm{H}), 2.66(\mathrm{dd}, J=13.8,8.2 \mathrm{~Hz}, 1 \mathrm{H}), 2.29(\mathrm{dd}, J=15.4,8.7 \mathrm{~Hz}, 1 \mathrm{H}), 2.15(\mathrm{dd}, J=15.4,3.5$ $\mathrm{Hz}, 1 \mathrm{H}), 2.08$ (qd, $J=8.4,6.2 \mathrm{~Hz}, 1 \mathrm{H}), 1.28(\mathrm{t}, J=7.1 \mathrm{~Hz}, 3 \mathrm{H})$.

${ }^{13}$ C NMR $\left(101 \mathrm{MHz}, \mathrm{CDCl}_{3}\right) \delta 171.56,166.16,147.13,138.73,129.10,128.76,126.79,122.93,81.05$, 71.20, 60.52, 51.99, 51.88, 49.17, 39.73, 37.60, 14.37 .

HRMS (ESI - TOF) m/z: Calcd for $\mathrm{C}_{19} \mathrm{H}_{24} \mathrm{NaO}_{5}[\mathrm{M}+\mathrm{Na}]^{+} 355.1516$, found 355.1503.

\section{v. Oxidative esterification of SI-7a and 8a}

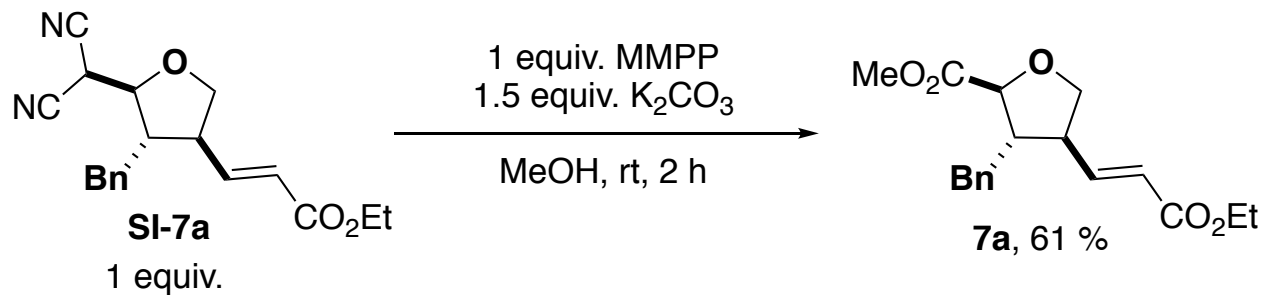

SI-7a (1 equiv., $10 \mathrm{mg}, 0.031 \mathrm{mmol})$ was dissolved in dry MeOH $(1 \mathrm{~mL})$ in a flame-dried Schlenk flask under $\mathrm{N}_{2} . \mathrm{K}_{2} \mathrm{CO}_{3}$ (1.5 equiv., $6 \mathrm{mg}, 0.046 \mathrm{mmol}$ ) and MMPP (magnesium monoperoxyphthalate $80 \%, 1$ equiv., $12 \mathrm{mg}, 0.031 \mathrm{mmol}$ ) were added sequentially in this order at room temperature. After $2 \mathrm{~h}$ at room temperature, $\mathrm{MeOH}$ is removed under reduced pressure. The residue is diluted with EtOAc and was filtered through a short pad of silica to remove MMPP solid byproducts. The filtrate was then concentrated down and $7 \mathbf{a}$ was obtained clean without further purification. $(6 \mathrm{mg}, 61 \%$, colorless oil, 8:1 d.r., major reported below).

${ }^{1}$ H NMR $(600 \mathrm{MHz}$, Chloroform- $d) \delta 7.28(\mathrm{~d}, J=7.6 \mathrm{~Hz}, 3 \mathrm{H}), 7.20(\mathrm{tt}, J=7.6,1.4 \mathrm{~Hz}, 1 \mathrm{H}), 7.16(\mathrm{~d}, J=$ $6.7 \mathrm{~Hz}, 2 \mathrm{H}), 6.65(\mathrm{dd}, J=15.6,9.0 \mathrm{~Hz}, 1 \mathrm{H}), 5.72(\mathrm{dd}, J=15.7,0.9 \mathrm{~Hz}, 1 \mathrm{H}), 4.24(\mathrm{~d}, J=7.5 \mathrm{~Hz}, 1 \mathrm{H}), 4.16$ 
$(\mathrm{q}, J=7.2 \mathrm{~Hz}, 2 \mathrm{H}), 4.07$ (dd, $J=8.8,7.4 \mathrm{~Hz}, 1 \mathrm{H}), 3.82(\mathrm{t}, J=8.9 \mathrm{~Hz}, 1 \mathrm{H}), 3.65(\mathrm{~s}, 3 \mathrm{H}), 2.89(\mathrm{~h}, J=7.0$ $\mathrm{Hz}, 2 \mathrm{H}), 2.81-2.74(\mathrm{~m}, 1 \mathrm{H}), 2.57(\mathrm{dq}, J=8.7,7.1 \mathrm{~Hz}, 1 \mathrm{H}), 1.27(\mathrm{t}, J=7.1 \mathrm{~Hz}, 3 \mathrm{H})$.

${ }^{13}$ C NMR $\left(151 \mathrm{MHz}, \mathrm{CDCl}_{3}\right) \delta 172.87,165.95,145.59,137.93,129.55,128.63,126.86,123.63,81.55$, $72.93,60.58,52.29,51.23,48.08,37.62,14.37$.

HRMS (ESI - TOF) m/z: Calcd for $\mathrm{C}_{18} \mathrm{H}_{22} \mathrm{NaO}_{5}[\mathrm{M}+\mathrm{Na}]^{+} 341.1359$, found 341.1360.

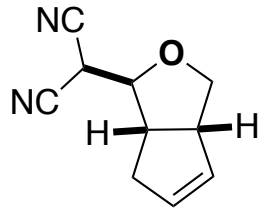

$8 \mathbf{a}$

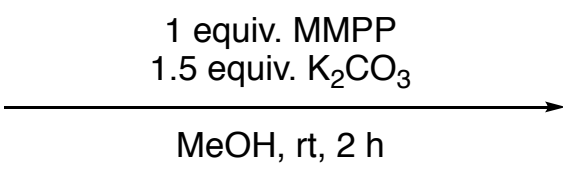

$\mathrm{MeOH}, \mathrm{rt}, 2 \mathrm{~h}$

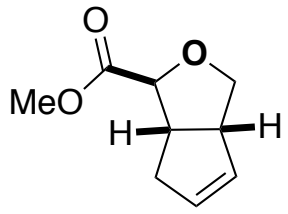

$8 b, 62 \%$

1 equiv.

8a (1 equiv., $10 \mathrm{mg}, 0.057 \mathrm{mmol})$ was dissolved in dry $\mathrm{MeOH}(1 \mathrm{~mL})$ in a flame-dried Schlenk flask under $\mathrm{N}_{2} . \mathrm{K}_{2} \mathrm{CO}_{3}$ (1.5 equiv., $12 \mathrm{mg}, 0.086 \mathrm{mmol}$ ) and MMPP (magnesium monoperoxyphthalate $80 \%$, 1 equiv., $22 \mathrm{mg}, 0.057 \mathrm{mmol}$ ) were added sequentially in this order at room temperature. After $2 \mathrm{~h}$ at room temperature, $\mathrm{MeOH}$ is removed under reduced pressure. The residue is diluted with EtOAc and was filtered through a short pad of silica to remove MMPP solid byproducts. The filtrate was then concentrated down and $\mathbf{8 b}$ was obtained clean without further purification. $(6 \mathrm{mg}, 62 \%$, yellowish oil, 7:1 d.r., major reported below).

${ }^{1}$ H NMR $(600 \mathrm{MHz}$, Chloroform- $d$ ) $\delta 5.73(\mathrm{dq}, J=4.6,2.2 \mathrm{~Hz}, 1 \mathrm{H}), 5.62-5.59(\mathrm{~m}, 1 \mathrm{H}), 4.18(\mathrm{dd}, J=8.7$, $7.1 \mathrm{~Hz}, 1 \mathrm{H}), 4.09(\mathrm{~d}, J=5.7 \mathrm{~Hz}, 1 \mathrm{H}), 3.79(\mathrm{dd}, J=8.8,3.1 \mathrm{~Hz}, 1 \mathrm{H}), 3.77(\mathrm{~s}, 3 \mathrm{H}), 3.46(\mathrm{dtd}, J=10.8,4.9$, $2.4 \mathrm{~Hz}, 1 \mathrm{H}), 2.98$ (tdd, $J=8.1,5.7,2.0 \mathrm{~Hz}, 1 \mathrm{H}), 2.71$ (ddq, $J=17.2,8.3,2.3 \mathrm{~Hz}, 1 \mathrm{H}), 2.45(\mathrm{dp}, J=17.3$, $2.2 \mathrm{~Hz}, 1 \mathrm{H})$.

${ }^{13}$ C NMR $\left(151 \mathrm{MHz}, \mathrm{CDCl}_{3}\right) \delta 173.39,132.04,130.62,84.67,73.57,52.19,51.95,46.40,38.62$.

HRMS (ESI - TOF) m/z: Calcd for $\mathrm{C}_{9} \mathrm{H}_{12} \mathrm{NaO}_{3}[\mathrm{M}+\mathrm{Na}]^{+}$191.0679, found 191.0671.

vi. Retro-Oxy-Michael addition on 3a
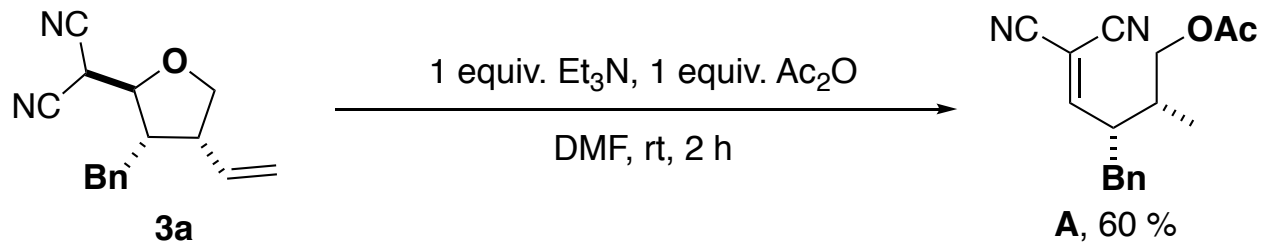

1 equiv.

A, $60 \%$

3a (1 equiv., $10 \mathrm{mg}, 0.04 \mathrm{mmol})$ was dissolved in DMF $(1 \mathrm{~mL})$ at room temperature in a $4 \mathrm{~mL}$ vial under air. $\mathrm{Et}_{3} \mathrm{~N}$ (1.1 equiv., $6 \mu \mathrm{L}, 0.043 \mathrm{mmol}$ ) was added and the reaction was stirred for $5 \mathrm{~min}$ before $\mathrm{Ac}_{2} \mathrm{O}(1.1$ equiv., $4 \mu \mathrm{L}, 0.043 \mathrm{mmol}$ ) was added. The reaction mixture was stirred at room temeprature for $2 \mathrm{~h}$. After completion, the reaction mixture was diluted with $2 \mathrm{M} \mathrm{HCl}_{(\mathrm{aq})}(3 \mathrm{~mL})$ and extracted with EtOAc three times $(3 \times 1 \mathrm{~mL})$. The combined organic layers were washed with $2 \mathrm{M} \mathrm{HCl}_{(\mathrm{aq})}(3 \mathrm{~mL})$, brine $(6 \mathrm{~mL})$ and dried over $\mathrm{Na}_{2} \mathrm{SO}_{4}$. The solvent was removed under reduced pressure and the residue was purified via sílica gel column chromatography yielding compound A (7 mg, $60 \%)$.

${ }^{1}$ H NMR (400 MHz, Chloroform- $d$ ) $\delta 7.39-7.29(\mathrm{~m}, 3 \mathrm{H}), 7.18-7.08(\mathrm{~m}, 3 \mathrm{H}), 5.72$ (ddd, $J=17.0,10.4$, $9.0 \mathrm{~Hz}, 1 \mathrm{H}), 5.39(\mathrm{dd}, J=10.3,1.2 \mathrm{~Hz}, 1 \mathrm{H}), 5.29$ (dt, $J=17.1,1.0 \mathrm{~Hz}, 1 \mathrm{H}), 4.19(\mathrm{dd}, J=11.4,5.6 \mathrm{~Hz}, 1 \mathrm{H})$, 4.00 (dd, $J=11.4,7.7 \mathrm{~Hz}, 1 \mathrm{H}), 3.38$ (dddd, $J=11.2,9.1,5.8,4.7 \mathrm{~Hz}, 1 \mathrm{H}), 3.03$ (dd, $J=13.8,5.9 \mathrm{~Hz}, 1 \mathrm{H}$ ), $2.80-2.76(\mathrm{~m}, 1 \mathrm{H}), 2.72(\mathrm{dd}, J=13.8,9.2 \mathrm{~Hz}, 1 \mathrm{H}), 2.12(\mathrm{~s}, 3 \mathrm{H})$. 
${ }^{13}$ C NMR $\left(101 \mathrm{MHz}, \mathrm{CDCl}_{3}\right) \delta 170.81,169.28,136.62,132.67,129.15,128.97,127.50,121.16,111.82$, $110.31,91.27,64.77,46.25,46.06,38.27,20.99$.

\section{3. ${ }^{1} \mathrm{H},{ }^{13} \mathrm{C}$ NMR \& mass spectrometry data}

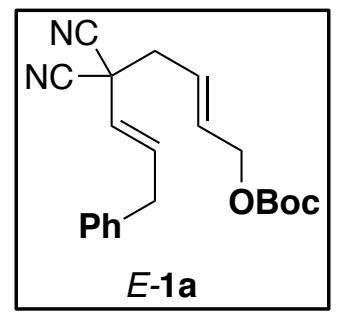

Synthesized according to Procedure B-1 (384 mg, $25 \%$ ).

Solvent system for column chromatography: Hex/EtOAc $10 \%$.

${ }^{1}$ H NMR (400 MHz, Chloroform- $d$ ): $\delta 7.33$ (tt, $\left.J=7.8,1.6 \mathrm{~Hz}, 2 \mathrm{H}\right), 7.29-7.25(\mathrm{~m}, 1 \mathrm{H}), 7.15(\mathrm{~d}, J=7.0$ $\mathrm{Hz}, 2 \mathrm{H}), 6.38(\mathrm{dt}, J=15.2,6.6 \mathrm{~Hz}, 1 \mathrm{H}), 5.90(\mathrm{dtt}, J=15.4,5.7,0.8 \mathrm{~Hz}, 1 \mathrm{H}), 5.79(\mathrm{dtt}, J=15.4,7.2,1.3$ $\mathrm{Hz}, 1 \mathrm{H}), 5.38(\mathrm{dt}, J=15.1,1.6 \mathrm{~Hz}, 1 \mathrm{H}), 4.57(\mathrm{dd}, J=5.5,0.8 \mathrm{~Hz}, 2 \mathrm{H}), 3.48(\mathrm{dd}, J=6.4,1.6 \mathrm{~Hz}, 2 \mathrm{H}), 2.75$ (dd, $J=7.2,0.7 \mathrm{~Hz}, 2 \mathrm{H}), 1.50(\mathrm{~s}, 9 \mathrm{H})$.

${ }^{13}$ C NMR $\left(101 \mathrm{MHz}, \mathrm{CDCl}_{3}\right): \delta 153.21,137.59,137.47,133.00,128.97,128.78,127.01,124.10,121.56$, $113.98,82.65,66.05,42.19,38.94,38.11,27.89$.

HRMS (ESI - TOF) m/z: Calcd for $\mathrm{C}_{21} \mathrm{H}_{24} \mathrm{~N}_{2} \mathrm{NaO}_{3}[\mathrm{M}+\mathrm{Na}]^{+} 375.1679$, found 375.1675.

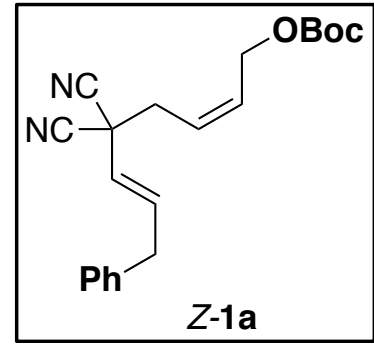

Synthesized according to Procedure A-1 (337 mg, $41 \%$ ).

Solvent system for column chromatography: Hex/EtOAc $10 \%$.

${ }^{1}$ H NMR $(600 \mathrm{MHz}$, Chloroform- $d$ ) $\delta 7.36-7.31(\mathrm{~m}, 2 \mathrm{H}), 7.30-7.26(\mathrm{~m}, 1 \mathrm{H}), 7.19-7.14(\mathrm{~m}, 3 \mathrm{H}), 6.40$ (dt, $J=15.2,6.6 \mathrm{~Hz}, 1 \mathrm{H}), 5.98(\mathrm{dtt}, J=11.2,6.9,1.4 \mathrm{~Hz}, 1 \mathrm{H}), 5.71(\mathrm{dtt}, J=10.8,7.7,1.4 \mathrm{~Hz}, 1 \mathrm{H}), 5.40$ $(\mathrm{dt}, J=15.2,1.7 \mathrm{~Hz}, 1 \mathrm{H}), 4.62(\mathrm{dd}, J=6.8,1.5 \mathrm{~Hz}, 2 \mathrm{H}), 3.48(\mathrm{dd}, J=6.5,1.7 \mathrm{~Hz}, 2 \mathrm{H}), 2.88(\mathrm{dd}, J=7.7$, $1.4 \mathrm{~Hz}, 2 \mathrm{H}), 1.48(\mathrm{~s}, 9 \mathrm{H})$.

${ }^{13}$ C NMR $\left(151 \mathrm{MHz}, \mathrm{CDCl}_{3}\right) \delta 153.34,137.56,131.30,128.98,128.80,127.03,124.29,121.51,114.02$, 82.78, 61.91, 38.69, 38.12, 37.39, 27.88.

HRMS (ESI - TOF) m/z: Calcd for $\mathrm{C}_{21} \mathrm{H}_{24} \mathrm{~N}_{2} \mathrm{NaO}_{3}[\mathrm{M}+\mathrm{Na}]^{+} 375.1679$, found 375.1675.

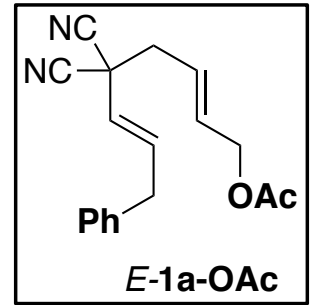

Synthesized according to Procedure B-1 with $\mathrm{Ac}_{2} \mathrm{O}$ instead of $\mathrm{Boc}_{2} \mathrm{O}(236 \mathrm{mg}, 49 \%)$. 
Solvent system for column chromatography: Hex/EtOAc $10 \%$.

${ }^{1}$ H NMR (400 MHz, Chloroform- $d$ ) $\delta 7.37-7.30(\mathrm{~m}, 2 \mathrm{H}), 7.29-7.26(\mathrm{~m}, 1 \mathrm{H}), 7.18-7.13(\mathrm{~m}, 2 \mathrm{H}), 6.38$ (dt, $J=15.2,6.6 \mathrm{~Hz}, 1 \mathrm{H}), 5.89$ (dtt, $J=15.3,5.6,1.1 \mathrm{~Hz}, 1 \mathrm{H}), 5.75$ (dtt, $J=15.6,7.3,1.4 \mathrm{~Hz}, 1 \mathrm{H}), 5.38$ $(\mathrm{dt}, J=15.2,1.7 \mathrm{~Hz}, 1 \mathrm{H}), 4.58(\mathrm{dd}, J=5.6,1.2 \mathrm{~Hz}, 2 \mathrm{H}), 3.49$ (dd, $J=6.6,1.6 \mathrm{~Hz}, 2 \mathrm{H}), 2.74(\mathrm{dd}, J=7.3$, $1.0 \mathrm{~Hz}, 2 \mathrm{H}), 2.08(\mathrm{~s}, 3 \mathrm{H})$.

${ }^{13}$ C NMR $\left(101 \mathrm{MHz}, \mathrm{CDCl}_{3}\right) \delta 170.67,137.59,137.47,133.20,128.96,128.77,127.03,123.79,121.57$, $113.98,63.69,42.19,39.05,38.12,21.00$.

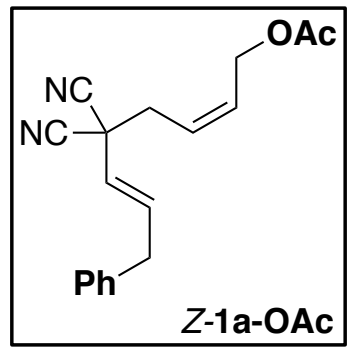

Synthesized according to Procedure A-1 with (Z)-4-bromobut-2-en-1-yl acetate (135 mg, 40 \%).

Solvent system for column chromatography: Hex/EtOAc $10 \%$.

${ }^{1}$ H NMR (400 MHz, Chloroform- $d$ ) $\delta 7.36-7.31(\mathrm{~m}, 2 \mathrm{H}), 7.29-7.26(\mathrm{~m}, 1 \mathrm{H}), 7.23-7.09$ (m, 2H), 6.40 $(\mathrm{dt}, J=15.2,6.6 \mathrm{~Hz}, 1 \mathrm{H}), 5.96(\mathrm{dtt}, J=11.0,6.9,1.4 \mathrm{~Hz}, 1 \mathrm{H}), 5.70(\mathrm{dtt}, J=10.7,7.8,1.4 \mathrm{~Hz}, 1 \mathrm{H}), 5.40$ $(\mathrm{dt}, J=15.2,1.7 \mathrm{~Hz}, 1 \mathrm{H}), 4.63(\mathrm{dd}, J=7.0,1.4 \mathrm{~Hz}, 2 \mathrm{H}), 3.49$ (dd, $J=6.6,1.6 \mathrm{~Hz}, 2 \mathrm{H}), 2.87(\mathrm{dd}, J=7.6$, $1.4 \mathrm{~Hz}, 2 \mathrm{H}), 2.06$ (s, 3H).

${ }^{13}$ C NMR $\left(101 \mathrm{MHz}, \mathrm{CDCl}_{3}\right) \delta 170.80,137.53,137.52,131.44,128.98,128.79,127.04,124.20,121.53$, $114.01,59.58,38.78,38.11,37.41,21.00$.

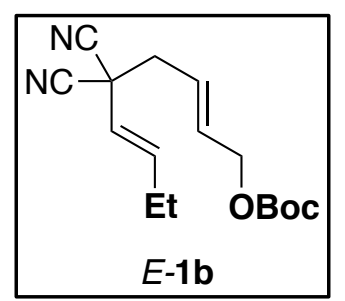

Synthesized according to Procedure B-1 (90 mg, $25 \%$ ).

Solvent system for column chromatography: Hex/EtOAc $10 \%$.

${ }^{1}$ H NMR (400 MHz, Chloroform- $d$ ) $\delta 6.24(\mathrm{dt}, J=15.3,6.4 \mathrm{~Hz}, 1 \mathrm{H}), 5.92(\mathrm{dtt}, J=15.4,5.5,1.0 \mathrm{~Hz}, 1 \mathrm{H})$, $5.80(\mathrm{dtt}, J=15.5,7.3,1.3 \mathrm{~Hz}, 1 \mathrm{H}), 5.36(\mathrm{dt}, J=15.3,1.7 \mathrm{~Hz}, 1 \mathrm{H}), 4.59(\mathrm{dq}, J=5.7,0.9 \mathrm{~Hz}, 2 \mathrm{H}), 2.74(\mathrm{dd}$, $J=7.2,1.0 \mathrm{~Hz}, 2 \mathrm{H}), 2.23-2.14(\mathrm{~m}, 2 \mathrm{H}), 1.49(\mathrm{~s}, 9 \mathrm{H}), 1.06(\mathrm{t}, J=7.4 \mathrm{~Hz}, 3 \mathrm{H})$.

${ }^{13} \mathbf{C}$ NMR $\left(101 \mathrm{MHz}, \mathrm{CDCl}_{3}\right) \delta 153.23,140.15,132.82,124.33,119.52,114.19,82.64,66.09,42.28,39.08$, $27.88,25.14,12.83$.

HRMS (ESI - TOF) m/z: Calcd for $\mathrm{C}_{16} \mathrm{H}_{22} \mathrm{~N}_{2} \mathrm{NaO}_{3}[\mathrm{M}+\mathrm{Na}]^{+} 313.1523$, found 313.1522 .

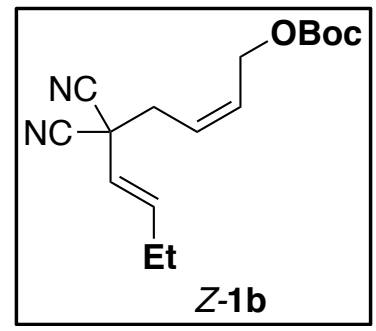


Synthesized according to Procedure A-1 (54 mg, $31 \%$ ).

Solvent system for column chromatography: Hex/EtOAc $10 \%$.

${ }^{1}$ H NMR (400 MHz, Chloroform- $d$ ) $\delta 6.25(\mathrm{dt}, J=15.3,6.3 \mathrm{~Hz}, 1 \mathrm{H}), 5.98(\mathrm{dtt}, J=11.2,6.9,1.4 \mathrm{~Hz}, 1 \mathrm{H})$, $5.72(\mathrm{dtt}, J=10.7,7.7,1.5 \mathrm{~Hz}, 1 \mathrm{H}), 5.38(\mathrm{dt}, J=15.3,1.7 \mathrm{~Hz}, 1 \mathrm{H}), 4.63(\mathrm{dd}, J=7.0,1.4 \mathrm{~Hz}, 2 \mathrm{H}), 2.87$ (dd, $J=7.7,1.4 \mathrm{~Hz}, 2 \mathrm{H}), 2.18$ (qdd, $J=7.5,6.3,1.7 \mathrm{~Hz}, 2 \mathrm{H}), 1.48(\mathrm{~s}, 9 \mathrm{H}), 1.05$ (t, $J=7.4 \mathrm{~Hz}, 3 \mathrm{H})$.

${ }^{13}$ C NMR $\left(101 \mathrm{MHz}, \mathrm{CDCl}_{3}\right) \delta 153.32,140.18,131.13,124.43,119.46,114.21,82.72,61.91,38.74,37.44$, $27.85,25.10,12.75$.

HRMS (ESI - TOF) m/z: Calcd for $\mathrm{C}_{16} \mathrm{H}_{22} \mathrm{~N}_{2} \mathrm{NaO}_{3}[\mathrm{M}+\mathrm{Na}]^{+} 313.1523$, found 313.1522.

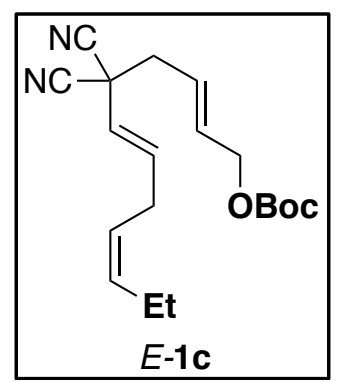

Synthesized according to Procedure B-1 (100 mg, $49 \%$ ).

Solvent system for column chromatography: Hex/EtOAc $10 \%$.

${ }^{1}$ H NMR $(400 \mathrm{MHz}$, Chloroform- $d) \delta 6.20(\mathrm{dt}, J=15.3,6.0 \mathrm{~Hz}, 1 \mathrm{H}), 5.91(\mathrm{dtt}, J=15.4,5.6,0.5 \mathrm{~Hz}, 1 \mathrm{H})$, $5.84-5.74(\mathrm{~m}, 1 \mathrm{H}), 5.56(\mathrm{dtt}, J=10.6,7.4,1.2 \mathrm{~Hz}, 1 \mathrm{H}), 5.39(\mathrm{dt}, J=15.2,1.8 \mathrm{~Hz}, 1 \mathrm{H}), 5.31(\mathrm{dtt}, J=10.7$, $7.3,1.7 \mathrm{~Hz}, 1 \mathrm{H}), 4.58(\mathrm{~d}, J=5.6 \mathrm{~Hz}, 2 \mathrm{H}), 2.89(\mathrm{t}, J=6.6 \mathrm{~Hz}, 2 \mathrm{H}), 2.73(\mathrm{~d}, J=7.1 \mathrm{~Hz}, 2 \mathrm{H}), 2.11-1.96(\mathrm{~m}$, 2H), $1.49(\mathrm{~s}, 9 \mathrm{H}), 0.98(\mathrm{t}, J=7.5 \mathrm{~Hz}, 3 \mathrm{H})$.

${ }^{13}$ C NMR (101 MHz, $\left.\mathrm{CDCl}_{3}\right) \delta 153.21,137.02,135.00,132.86,124.24,123.51,120.51,114.09,82.63$, 66.07, 42.24, 39.06, 29.46, 27.88, 20.67, 14.26.

HRMS (ESI - TOF) m/z: Calcd for $\mathrm{C}_{19} \mathrm{H}_{26} \mathrm{~N}_{2} \mathrm{NaO}_{3}[\mathrm{M}+\mathrm{Na}]^{+}$353.1836, fouond 353.1827.

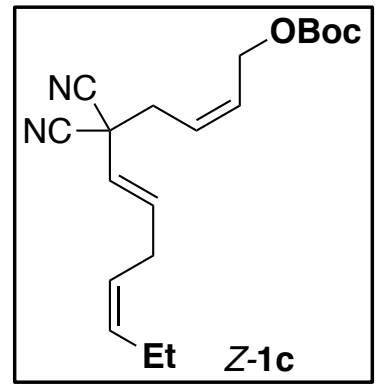

Synthesized according to Procedure A-2 (51 mg, $12 \%)$.

Solvent system for column chromatography: Hex/EtOAc $10 \%$.

${ }^{1}$ H NMR $(400 \mathrm{MHz}$, Chloroform- $d$ ) $\delta 6.22(\mathrm{dt}, J=15.2,6.1 \mathrm{~Hz}, 1 \mathrm{H}), 5.98(\mathrm{dtt}, J=11.0,6.8,1.4 \mathrm{~Hz}, 1 \mathrm{H})$, $5.71(\mathrm{dtt}, J=10.7,7.7,1.5 \mathrm{~Hz}, 1 \mathrm{H}), 5.56(\mathrm{dtt}, J=10.3,7.3,1.5 \mathrm{~Hz}, 1 \mathrm{H}), 5.41(\mathrm{dt}, J=15.3,1.8 \mathrm{~Hz}, 1 \mathrm{H})$, $5.31(\mathrm{dtt}, J=10.6,7.3,1.6 \mathrm{~Hz}, 1 \mathrm{H}), 4.63(\mathrm{dd}, J=6.9,1.4 \mathrm{~Hz}, 2 \mathrm{H}), 2.94-2.84$ (m, 4H), 2.03 (pd, $J=7.5$, $1.6 \mathrm{~Hz}, 3 \mathrm{H}), 1.48(\mathrm{~s}, 9 \mathrm{H}), 0.97(\mathrm{t}, J=7.5 \mathrm{~Hz}, 3 \mathrm{H})$.

${ }^{13}$ C NMR $\left(101 \mathrm{MHz}, \mathrm{CDCl}_{3}\right) \delta 153.31,137.09,134.99,131.20,124.34,123.46,120.46,114.11,82.73$, 61.91, 38.74, 37.41, 29.44, 27.85, 20.66, 14.23.

HRMS (ESI - TOF) m/z: Calcd for $\mathrm{C}_{19} \mathrm{H}_{26} \mathrm{~N}_{2} \mathrm{NaO}_{3}[\mathrm{M}+\mathrm{Na}]^{+}$353.1836, fouond 353.1827. 


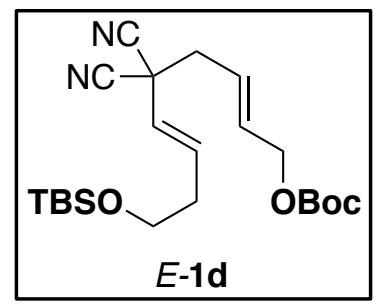

Synthesized according to Procedure B-1 (122 mg, $58 \%$ ).

Solvent system for column chromatography: Hex/EtOAc $5 \% \rightarrow 10 \%$.

${ }^{1}$ H NMR (400 MHz, Chloroform- $d$ ) $\delta 6.23(\mathrm{dt}, J=15.3,7.0 \mathrm{~Hz}, 1 \mathrm{H}), 5.93(\mathrm{dtt}, J=15.4,5.5,1.0 \mathrm{~Hz}, 1 \mathrm{H})$, $5.80(\mathrm{dtt}, J=15.4,7.2,1.0 \mathrm{~Hz}, 1 \mathrm{H}), 5.46(\mathrm{dt}, J=15.3,1.5 \mathrm{~Hz}, 1 \mathrm{H}), 4.59(\mathrm{dd}, J=5.5,1.2 \mathrm{~Hz}, 2 \mathrm{H}), 3.70(\mathrm{t}$, $J=6.2 \mathrm{~Hz}, 2 \mathrm{H}), 2.74(\mathrm{~d}, J=7.6 \mathrm{~Hz}, 2 \mathrm{H}), 2.36(\mathrm{dtd}, J=7.6,6.2,1.5 \mathrm{~Hz}, 2 \mathrm{H}), 1.49(\mathrm{~s}, 9 \mathrm{H}), 0.89(\mathrm{~s}, 9 \mathrm{H})$, $0.06(\mathrm{~s}, 6 \mathrm{H})$.

${ }^{13}$ C NMR $\left(101 \mathrm{MHz}, \mathrm{CDCl}_{3}\right) \delta 153.21,135.68,132.86,128.12,124.29,121.98,114.02,82.63,66.08$, $62.41,61.60,42.27,39.21,35.36,27.89,26.00,18.39,-5.20$

HRMS (ESI - TOF) m/z: Calcd for $\mathrm{C}_{22} \mathrm{H}_{36} \mathrm{~N}_{2} \mathrm{NaO}_{4} \mathrm{Si}[\mathrm{M}+\mathrm{Na}]^{+}$443.2337, found 443.2335.

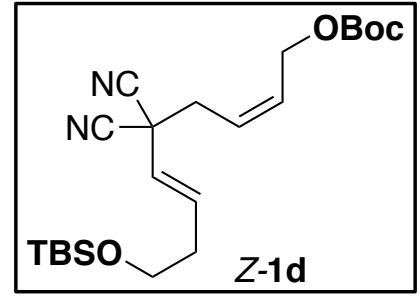

Synthesized according to Procedure A-2 (60 mg, $30 \% *$ ).

*Note: Z-1d was isolated along with $10 \%$ SM, inseparable.

Solvent system for column chromatography: Hex/EtOAc $5 \% \rightarrow 10 \%$.

${ }^{1}$ H NMR $(400 \mathrm{MHz}$, Chloroform- $d$ ) $\delta 6.24$ (dt, $J=15.3,7.0 \mathrm{~Hz}, 1 \mathrm{H}), 5.98$ (dtt, $\left.J=11.2,6.9,1.4 \mathrm{~Hz}, 1 \mathrm{H}\right)$, $5.73(\mathrm{dtt}, J=11.1,7.5,1.4 \mathrm{~Hz}, 1 \mathrm{H}), 5.48(\mathrm{dt}, J=15.3,1.5 \mathrm{~Hz}, 1 \mathrm{H}), 4.64(\mathrm{dd}, J=6.9,1.4 \mathrm{~Hz}, 2 \mathrm{H}), 3.70(\mathrm{t}$, $J=6.2 \mathrm{~Hz}, 2 \mathrm{H}), 2.88$ (dd, $J=7.7,1.4 \mathrm{~Hz}, 2 \mathrm{H}), 2.41-2.31(\mathrm{~m}, 2 \mathrm{H}), 1.48(\mathrm{~s}, 9 \mathrm{H}), 0.89$ (s, 9H), 0.06 (s, 6H). ${ }^{13}$ C NMR $\left(101 \mathrm{MHz}, \mathrm{CDCl}_{3}\right) \delta 153.33,135.82,131.19,124.43,121.93,114.07,82.74,61.91,61.62,38.88$, 37.42, 35.38, 27.88, 26.01, 18.40, -5.20.

HRMS (ESI - TOF) m/z: Calcd for $\mathrm{C}_{22} \mathrm{H}_{36} \mathrm{~N}_{2} \mathrm{NaO}_{4} \mathrm{Si}[\mathrm{M}+\mathrm{Na}]^{+}$443.2337, found 443.2335.

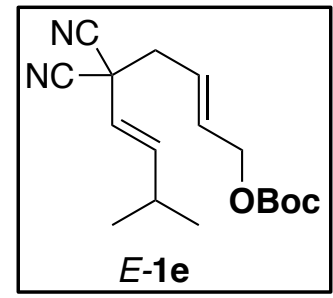

Synthesized according to Procedure B-1 (97 mg, $30 \%$ ).

Solvent system for column chromatography: Hex/EtOAc $10 \%$.

${ }^{1}$ H NMR (400 MHz, Chloroform- $d$ ) $\delta 6.16(\mathrm{dd}, J=15.3,6.7 \mathrm{~Hz}, 1 \mathrm{H}), 5.92(\mathrm{dt}, J=15.5,5.5 \mathrm{~Hz}, 1 \mathrm{H}), 5.79$ $(\mathrm{dtt}, J=15.4,7.1,1.2 \mathrm{~Hz}, 1 \mathrm{H}), 5.32(\mathrm{dd}, J=15.3,1.4 \mathrm{~Hz}, 1 \mathrm{H}), 4.59(\mathrm{~d}, J=5.5 \mathrm{~Hz}, 2 \mathrm{H}), 2.74(\mathrm{~d}, J=7.3$ $\mathrm{Hz}, 2 \mathrm{H}), 2.47-2.37(\mathrm{~m}, 1 \mathrm{H}), 1.49(\mathrm{~s}, 9 \mathrm{H}), 1.05(\mathrm{~d}, J=6.7 \mathrm{~Hz}, 6 \mathrm{H})$.

${ }^{13} \mathbf{C} \mathbf{N M R}\left(101 \mathrm{MHz}, \mathrm{CDCl}_{3}\right) \delta 153.22,145.16,132.82,124.31,117.96,114.21,82.62,66.07,42.31,39.05$, $31.00,27.88,21.78$.

HRMS (ESI - TOF) m/z: Calcd for $\mathrm{C}_{17} \mathrm{H}_{24} \mathrm{~N}_{2} \mathrm{NaO}_{3}[\mathrm{M}+\mathrm{Na}]^{+} 327.1679$, found 327.1662. 


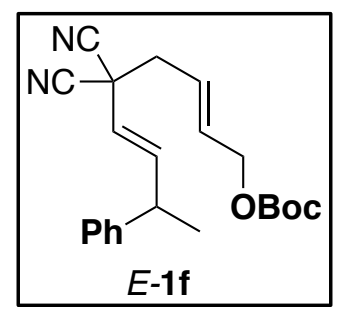

Synthesized according to Procedure B-1 (186 mg, 50 \%).

Solvent system for column chromatography: Hex/EtOAc $10 \%$.

${ }^{1}$ H NMR (400 MHz, Chloroform- $d$ ) $\delta 7.34(\mathrm{t}, J=7.5 \mathrm{~Hz}, 2 \mathrm{H}), 7.29-7.26(\mathrm{~m}, 1 \mathrm{H}), 7.17(\mathrm{~d}, J=7.3 \mathrm{~Hz}$, $2 \mathrm{H}), 6.37(\mathrm{dd}, J=15.3,6.5 \mathrm{~Hz}, 1 \mathrm{H}), 5.89(\mathrm{dt}, J=15.2,5.7 \mathrm{~Hz}, 1 \mathrm{H}), 5.77(\mathrm{dtt}, J=15.3,7.3,1.4 \mathrm{~Hz}, 1 \mathrm{H})$, $5.33(\mathrm{dd}, J=15.1,1.6 \mathrm{~Hz}, 1 \mathrm{H}), 4.56(\mathrm{~d}, J=5.2 \mathrm{~Hz}, 2 \mathrm{H}), 3.69-3.51(\mathrm{~m}, 1 \mathrm{H}), 2.74(\mathrm{~d}, J=7.2 \mathrm{~Hz}, 2 \mathrm{H}), 1.50$ (s, 9H), $1.42(\mathrm{~d}, J=7.0 \mathrm{~Hz}, 3 \mathrm{H})$.

${ }^{13}$ C NMR $\left(101 \mathrm{MHz}, \mathrm{CDCl}_{3}\right) \delta 153.22,143.19,142.97,132.98,128.96,127.30,127.07,124.13,119.66$, 114.06, 114.02, 82.64, 66.04, 42.25, 41.90, 38.96, 27.89, 20.76.

HRMS (ESI - TOF) m/z: Calcd for $\mathrm{C}_{22} \mathrm{H}_{26} \mathrm{~N}_{2} \mathrm{NaO}_{3}[\mathrm{M}+\mathrm{Na}]^{+} 389.1836$, found 389.1842.

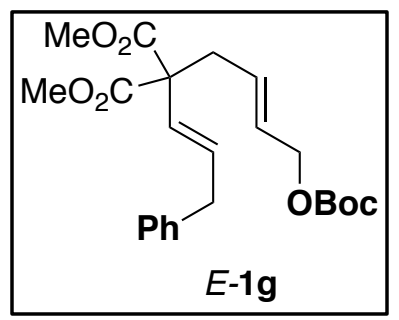

Synthesized according to Procedure B-2 (73 mg, $43 \%$ ).

Solvent system for column chromatography: Hex/EtOAc $10 \%$.

${ }^{1}$ H NMR $(400 \mathrm{MHz}$, Chloroform- $d$ ) $\delta 7.32-7.27(\mathrm{~m}, 2 \mathrm{H}), 7.19(\mathrm{dd}, J=7.3,1.2 \mathrm{~Hz}, 1 \mathrm{H}), 7.16-7.12(\mathrm{~m}$, 2H), $5.99(\mathrm{dt}, J=16.0,1.5 \mathrm{~Hz}, 1 \mathrm{H}), 5.71(\mathrm{dt}, J=16.0,6.9 \mathrm{~Hz}, 1 \mathrm{H}), 5.66-5.61(\mathrm{~m}, 2 \mathrm{H}), 4.44(\mathrm{~d}, J=4.7$ $\mathrm{Hz}, 2 \mathrm{H}), 3.73(\mathrm{~s}, 6 \mathrm{H}), 3.42(\mathrm{~d}, J=6.7 \mathrm{~Hz}, 2 \mathrm{H}), 2.80(\mathrm{~d}, J=5.5 \mathrm{~Hz}, 1 \mathrm{H}), 1.48(\mathrm{~s}, 9 \mathrm{H})$.

${ }^{13}$ C NMR $\left(101 \mathrm{MHz}, \mathrm{CDCl}_{3}\right) \delta 170.66,153.40,139.68,132.08,129.85,128.63,128.61,128.34,127.65$, 126.36, 82.21, 67.12, 59.48, 52.86, 39.14, 38.52, 27.92.

HRMS (ESI - TOF) m/z: Calcd for $\mathrm{C}_{23} \mathrm{H}_{30} \mathrm{NaO}_{7}[\mathrm{M}+\mathrm{Na}]^{+} 441.1884$, found 441.1888 .

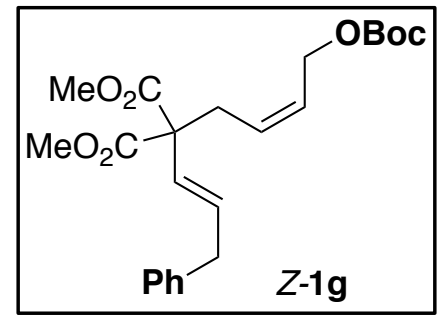

Synthesized according to Procedure A-2 (112 mg, $46 \%$ ).

Solvent system for column chromatography: Hex/EtOAc $10 \%$.

${ }^{1}$ H NMR (600 MHz, Chloroform- $\left.d\right) \delta 7.30-7.26(\mathrm{~m}, 2 \mathrm{H}), 7.19(\mathrm{tt}, J=7.5,1.2 \mathrm{~Hz}, 1 \mathrm{H}), 7.14(\mathrm{~d}, J=7.0$ $\mathrm{Hz}, 2 \mathrm{H}), 6.00(\mathrm{dt}, J=16.0,1.5 \mathrm{~Hz}, 1 \mathrm{H}), 5.74(\mathrm{dt}, J=16.0,6.9 \mathrm{~Hz}, 1 \mathrm{H}), 5.66(\mathrm{dtt}, J=11.1,6.8,1.5 \mathrm{~Hz}$, $1 \mathrm{H}), 5.52(\mathrm{dtt}, J=10.7,7.6,1.5 \mathrm{~Hz}, 1 \mathrm{H}), 4.57(\mathrm{dd}, J=6.7,1.5 \mathrm{~Hz}, 2 \mathrm{H}), 3.73(\mathrm{~s}, 6 \mathrm{H}), 3.43(\mathrm{~d}, J=6.7 \mathrm{~Hz}$, $2 \mathrm{H}), 2.86(\mathrm{dd}, J=7.5,1.6 \mathrm{~Hz}, 2 \mathrm{H}), 1.47(\mathrm{~s}, 9 \mathrm{H})$. 
${ }^{13}$ C NMR $\left(151 \mathrm{MHz}, \mathrm{CDCl}_{3}\right) \delta 170.64,153.52,139.61,132.21,128.62,128.22,127.53,127.17,126.36$, 82.27, 62.64, 59.24, 52.93, 39.12, 33.62, 27.91.

HRMS (ESI - TOF) m/z: Calcd for $\mathrm{C}_{23} \mathrm{H}_{30} \mathrm{NaO}_{7}[\mathrm{M}+\mathrm{Na}]^{+} 441.1884$, found 441.1888.

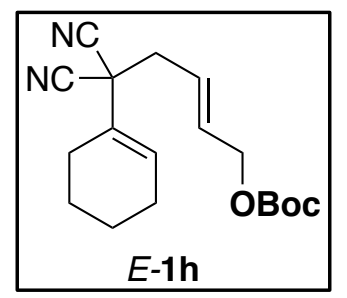

Synthesized according to Procedure B-1 (73 mg, $22 \%$ ).

Solvent system for column chromatography: Hex/EtOAc $10 \%$.

${ }^{1}$ H NMR $(400 \mathrm{MHz}$, Chloroform- $d$ ) $\delta 6.21(\mathrm{tt}, J=3.8,1.5 \mathrm{~Hz}, 1 \mathrm{H}), 5.91(\mathrm{dtt}, J=15.3,5.6,1.0 \mathrm{~Hz}, 1 \mathrm{H})$, $5.76(\mathrm{dtt}, J=15.6,7.3,1.3 \mathrm{~Hz}, 1 \mathrm{H}), 4.58(\mathrm{dd}, J=5.7,1.2 \mathrm{~Hz}, 2 \mathrm{H}), 2.76(\mathrm{dd}, J=7.2,1.0 \mathrm{~Hz}, 2 \mathrm{H}), 2.22-$ $2.07(\mathrm{~m}, 4 \mathrm{H}), 1.77-1.69(\mathrm{~m}, 2 \mathrm{H}), 1.66-1.58(\mathrm{~m}, 2 \mathrm{H}), 1.49(\mathrm{~s}, 9 \mathrm{H})$.

${ }^{13}$ C NMR $\left(101 \mathrm{MHz}, \mathrm{CDCl}_{3}\right) \delta 153.24,132.32,130.19,127.48,124.83,114.29,82.58,66.16,43.73,40.07$, $27.89,25.38,24.70,22.38,21.43$.

HRMS (ESI - TOF) m/z: Calcd for $\mathrm{C}_{18} \mathrm{H}_{24} \mathrm{~N}_{2} \mathrm{NaO}_{3}[\mathrm{M}+\mathrm{Na}]^{+} 339.1679$, found 339.1674.

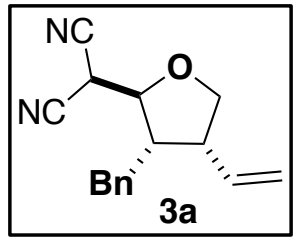

Synthesized according to Procedure C-1 (48 mg, $67 \%$, 5:1 d.r.).

Solvent system for column chromatography: Hex/EtOAc $20 \%$.

${ }^{1}$ H NMR $(400 \mathrm{MHz}$, Chloroform- $d$ ) $\delta 7.38-7.33(\mathrm{~m}, 2 \mathrm{H}), 7.32-7.27(\mathrm{~m}, 1 \mathrm{H}), 7.19$ (dd, $J=6.8,1.6 \mathrm{~Hz}$, 2H), 5.84 (ddd, $J=17.0,10.4,9.1 \mathrm{~Hz}, 1 \mathrm{H}), 5.29-5.19(\mathrm{~m}, 2 \mathrm{H}), 4.20$ (dd, $J=8.8,5.5 \mathrm{~Hz}, 1 \mathrm{H}), 4.06$ (dd, $J$ $=7.7,3.0 \mathrm{~Hz}, 1 \mathrm{H}), 3.90(\mathrm{dd}, J=8.8,3.6 \mathrm{~Hz}, 1 \mathrm{H}), 3.12(\mathrm{dtd}, J=9.3,6.0,3.7 \mathrm{~Hz}, 1 \mathrm{H}), 2.99(\mathrm{dd}, J=13.6$, $5.4 \mathrm{~Hz}, 1 \mathrm{H}), 2.87$ (d, $J=2.9 \mathrm{~Hz}, 1 \mathrm{H}), 2.72-2.65$ (m, 1H), 2.54 (dd, $J=13.6,10.5 \mathrm{~Hz}, 1 \mathrm{H})$.

${ }^{13} \mathrm{C}$ NMR $\left(101 \mathrm{MHz}, \mathrm{CDCl}_{3}\right) \delta 138.47,134.49,129.45,128.60,127.50,118.72,111.58,110.88,80.76$, 73.95, 48.16, 47.34, 34.86, 28.82.

HRMS (ESI - TOF) m/z: Calcd for $\mathrm{C}_{16} \mathrm{H}_{15} \mathrm{~N}_{2} \mathrm{O}$ [M-H] $]^{-} 251.1190$, found 251.1182.

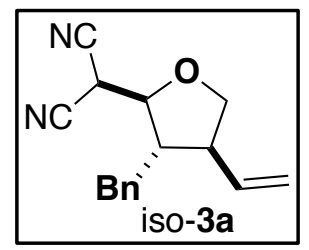

Synthesized according to Procedure C-1 (52 mg, 73 \%, 8:1 d.r.), (Scale-up: $180 \mathrm{mg}, 84$ \%, 8:1 d.r.). Solvent system for column chromatography: Hex/EtOAc $20 \%$.

${ }^{1}$ H NMR $(400 \mathrm{MHz}$, Chloroform- $d$ ) $\delta 7.39-7.33(\mathrm{~m}, 2 \mathrm{H}), 7.33-7.27(\mathrm{~m}, 1 \mathrm{H}), 7.18(\mathrm{dd}, J=6.9,1.7 \mathrm{~Hz}$, 2H), 5.73 (ddd, $J=17.0,10.2,8.5 \mathrm{~Hz}, 1 \mathrm{H}), 5.28-5.20$ (m, 2H), 4.12 (dd, $J=8.7,7.7 \mathrm{~Hz}, 1 \mathrm{H}), 4.05$ (dd, $J$ $=8.6,2.5 \mathrm{~Hz}, 1 \mathrm{H}), 3.83(\mathrm{dd}, J=10.1,8.7 \mathrm{~Hz}, 1 \mathrm{H}), 3.17(\mathrm{dd}, J=13.7,4.5 \mathrm{~Hz}, 1 \mathrm{H}), 2.77(\mathrm{tt}, J=10.0,8.0$ $\mathrm{Hz}, 1 \mathrm{H}), 2.66$ (d, $J=2.5 \mathrm{~Hz}, 1 \mathrm{H}), 2.49$ (dd, $J=13.7,10.3 \mathrm{~Hz}, 1 \mathrm{H}), 2.28$ (tdd, $J=10.2,8.5,4.4 \mathrm{~Hz}, 1 \mathrm{H})$. 
${ }^{13}$ C NMR $\left(101 \mathrm{MHz}, \mathrm{CDCl}_{3}\right) \delta 138.12,134.83,129.56,128.62,127.69,119.29,111.59,110.95,81.96$, 73.43, 51.10, 50.36, 36.77, 28.72.

HRMS (ESI - TOF) m/z: Calcd for $\mathrm{C}_{16} \mathrm{H}_{15} \mathrm{~N}_{2} \mathrm{O}[\mathrm{M}-\mathrm{H}]^{-} 251.1190$, found 251.1182.

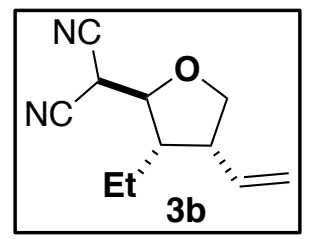

Synthesized according to Procedure C-1 (12 mg, $92 \%, 19: 1$ d.r.).

Solvent system for column chromatography: Hex/EtOAc $20 \%$.

${ }^{1}$ H NMR $(400 \mathrm{MHz}$, Chloroform- $d) \delta 5.74(\mathrm{ddd}, J=16.8,10.6,9.2 \mathrm{~Hz}, 1 \mathrm{H}), 5.21(\mathrm{dt}, J=2.3,1.0 \mathrm{~Hz}, 1 \mathrm{H})$, $5.20-5.16(\mathrm{~m}, 1 \mathrm{H}), 4.14(\mathrm{dd}, J=8.7,5.5 \mathrm{~Hz}, 1 \mathrm{H}), 4.07(\mathrm{dd}, J=7.3,4.0 \mathrm{~Hz}, 1 \mathrm{H}), 3.89(\mathrm{~d}, J=4.0 \mathrm{~Hz}, 1 \mathrm{H})$, $3.84(\mathrm{dd}, J=8.7,3.8 \mathrm{~Hz}, 1 \mathrm{H}), 3.07(\mathrm{dtd}, J=9.4,6.0,3.7 \mathrm{~Hz}, 1 \mathrm{H}), 2.26(\mathrm{p}, J=7.3 \mathrm{~Hz}, 1 \mathrm{H}), 1.56-1.45(\mathrm{~m}$, $2 \mathrm{H}), 1.00$ (t, $J=7.3 \mathrm{~Hz}, 3 \mathrm{H})$.

${ }^{13}$ C NMR $\left(101 \mathrm{MHz}, \mathrm{CDCl}_{3}\right) \delta 134.08,118.30,111.57,111.02,80.71,74.07,48.93,46.34,28.77,21.07$, 12.21, 1.16 .

HRMS (ESI - TOF) m/z: Calcd for $\mathrm{C}_{11} \mathrm{H}_{13} \mathrm{~N}_{2} \mathrm{O}[\mathrm{M}-\mathrm{H}]^{-}$189.1033, found 189.1023.

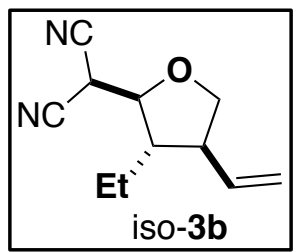

Synthesized according to Procedure C-1 (18 mg, $92 \%, 17: 1$ d.r.).

Solvent system for column chromatography: Hex/EtOAc $10 \%$.

${ }^{1}$ H NMR (400 MHz, Chloroform- $d$ ) $\delta 5.72(\mathrm{ddd}, J=17.1,10.2,8.5 \mathrm{~Hz}, 1 \mathrm{H}), 5.18(\mathrm{dt}, J=17.1,1.2 \mathrm{~Hz}$, $1 \mathrm{H}), 5.14(\mathrm{dd}, J=10.2,1.4 \mathrm{~Hz}, 1 \mathrm{H}), 4.11-4.02(\mathrm{~m}, 2 \mathrm{H}), 3.89$ (d, $J=4.1 \mathrm{~Hz}, 1 \mathrm{H}), 3.80$ (t, $J=9.1 \mathrm{~Hz}, 1 \mathrm{H})$, $2.73(\mathrm{q}, J=8.1 \mathrm{~Hz}, 1 \mathrm{H}), 2.07-1.97(\mathrm{~m}, 1 \mathrm{H}), 1.64$ (qdd, $J=7.4,6.3,3.9 \mathrm{~Hz}, 2 \mathrm{H}), 1.01$ (t, $J=7.5 \mathrm{~Hz}, 3 \mathrm{H})$. ${ }^{13} \mathrm{C}$ NMR $\left(101 \mathrm{MHz}, \mathrm{CDCl}_{3}\right) \delta 136.18,118.15,111.58,111.07,81.78,73.67,50.56,50.48,28.88,24.20$, 11.75 .

HRMS (ESI - TOF) m/z: Calcd for $\mathrm{C}_{11} \mathrm{H}_{13} \mathrm{~N}_{2} \mathrm{O}[\mathrm{M}-\mathrm{H}]^{-}$189.1033, found 189.1023 .

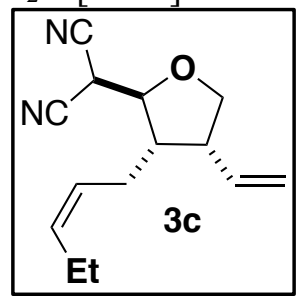

Synthesized according to Procedure C-1 (25 mg, 72\%, 7:1 d.r.).

Solvent system for column chromatography: Hex/EtOAc $10 \%$.

${ }^{1}$ H NMR (400 MHz, Chloroform- $d$ ) $\delta 5.76(\mathrm{ddd}, J=17.0,10.4,8.9 \mathrm{~Hz}, 1 \mathrm{H}), 5.54(\mathrm{dtt}, J=10.5,7.3,1.5$ $\mathrm{Hz}, 1 \mathrm{H}), 5.28(\mathrm{dtt}, J=10.8,6.5,1.7 \mathrm{~Hz}, 1 \mathrm{H}), 5.21(\mathrm{dd}, J=5.5,0.9 \mathrm{~Hz}, 1 \mathrm{H}), 5.18(\mathrm{dt}, J=14.1,1.1 \mathrm{~Hz}, 1 \mathrm{H})$, $4.17(\mathrm{dd}, J=8.7,5.8 \mathrm{~Hz}, 1 \mathrm{H}), 4.04(\mathrm{dd}, J=6.9,3.7 \mathrm{~Hz}, 1 \mathrm{H}), 3.92(\mathrm{~d}, J=3.7 \mathrm{~Hz}, 1 \mathrm{H}), 3.85(\mathrm{dd}, J=8.7,4.5$ $\mathrm{Hz}, 1 \mathrm{H}), 3.14-3.03(\mathrm{~m}, 1 \mathrm{H}), 2.39(\mathrm{dq}, J=8.3,7.1 \mathrm{~Hz}, 1 \mathrm{H}), 2.28-2.13(\mathrm{~m}, 2 \mathrm{H}), 2.10-1.99(\mathrm{~m}, 3 \mathrm{H}), 0.98$ (t, $J=7.5 \mathrm{~Hz}, 3 \mathrm{H})$. 
${ }^{13}$ C NMR $\left(101 \mathrm{MHz}, \mathrm{CDCl}_{3}\right) \delta 135.28,134.07,124.94,118.54,111.63,111.05,80.94,73.74,46.84,46.76$, 29.10, 26.19, 20.96, 14.21.

HRMS (ESI - TOF) m/z: Calcd for $\mathrm{C}_{14} \mathrm{H}_{17} \mathrm{~N}_{2} \mathrm{O}[\mathrm{M}-\mathrm{H}]^{-}$229.1335, found 229.1339.

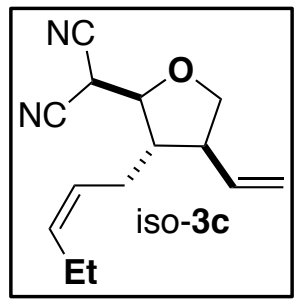

Synthesized according to Procedure C-1 (15 mg, $71 \%, 7: 1$ d.r.). Solvent system for column chromatography: Hex/EtOAc $10 \%$.

${ }^{1}$ H NMR (400 MHz, Chloroform- $d$ ) $\delta 5.69(\mathrm{ddd}, J=17.0,10.2,8.5 \mathrm{~Hz}, 1 \mathrm{H}), 5.57(\mathrm{dtt}, J=11.0,7.3,1.5$ $\mathrm{Hz}, 1 \mathrm{H}), 5.31(\mathrm{dtt}, J=10.6,6.7,1.3 \mathrm{~Hz}, 1 \mathrm{H}), 5.23-5.14(\mathrm{~m}, 2 \mathrm{H}), 4.09(\mathrm{dd}, J=8.7,7.7 \mathrm{~Hz}, 1 \mathrm{H}), 4.03(\mathrm{dd}$, $J=8.2,3.2 \mathrm{~Hz}, 1 \mathrm{H}), 3.97(\mathrm{~d}, J=3.2 \mathrm{~Hz}, 1 \mathrm{H}), 3.81(\mathrm{dd}, J=9.8,8.7 \mathrm{~Hz}, 1 \mathrm{H}), 2.78-2.66(\mathrm{~m}, 1 \mathrm{H}), 2.43-$ $2.33(\mathrm{~m}, 1 \mathrm{H}), 2.24(\mathrm{dtd}, J=14.4,8.6,1.2 \mathrm{~Hz}, 1 \mathrm{H}), 2.10-2.02(\mathrm{~m}, 3 \mathrm{H}), 0.98(\mathrm{t}, J=7.5 \mathrm{~Hz}, 3 \mathrm{H})$.

${ }^{13}$ C NMR $\left(101 \mathrm{MHz}, \mathrm{CDCl}_{3}\right) \delta 135.89,135.24,124.19,118.85,111.66,111.06,81.75,73.41,50.51,48.74$, 29.08, 27.72, 20.87, 14.23.

HRMS (ESI - TOF) m/z: Calcd for $\mathrm{C}_{14} \mathrm{H}_{17} \mathrm{~N}_{2} \mathrm{O}[\mathrm{M}-\mathrm{H}]^{-}$229.1335, found 229.1339.

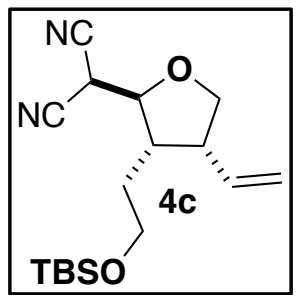

Synthesized according to Procedure C-1 (24 mg, $63 \%$, 16:1 d.r.).

Solvent system for column chromatography: Hex/EtOAc $10 \%$.

${ }^{1}$ H NMR (400 MHz, Chloroform- $d$ ) $\delta 5.74$ (ddd, $\left.J=17.0,10.4,9.4 \mathrm{~Hz}, 1 \mathrm{H}\right), 5.21-5.11(\mathrm{~m}, 2 \mathrm{H}), 4.44(\mathrm{~d}$, $J=2.7 \mathrm{~Hz}, 1 \mathrm{H}), 4.17(\mathrm{dd}, J=8.8,5.2 \mathrm{~Hz}, 1 \mathrm{H}), 4.13(\mathrm{dd}, J=8.2,2.7 \mathrm{~Hz}, 1 \mathrm{H}), 3.85(\mathrm{dd}, J=8.7,2.8 \mathrm{~Hz}$, 1H), 3.77 (dt, $J=10.9,4.9 \mathrm{~Hz}, 1 \mathrm{H}), 3.64$ (ddd, $J=10.7,8.8,3.7 \mathrm{~Hz}, 1 \mathrm{H}), 3.08-2.93(\mathrm{~m}, 1 \mathrm{H}), 2.54-2.42$ $(\mathrm{m}, 1 \mathrm{H}), 1.76(\mathrm{dtd}, J=14.4,5.3,3.7 \mathrm{~Hz}, 1 \mathrm{H}), 1.55(\mathrm{ddt}, J=14.4,8.8,4.6 \mathrm{~Hz}, 1 \mathrm{H}), 0.90(\mathrm{~s}, 9 \mathrm{H}), 0.07$ (s, $6 \mathrm{H})$.

${ }^{13}$ C NMR $\left(101 \mathrm{MHz}, \mathrm{CDCl}_{3}\right) \delta 135.02,118.15,112.17,111.55,81.32,73.95,62.06,47.94,45.19,31.78$, 28.51, 26.05, 18.44, -5.21, -5.30.

HRMS (ESI - TOF) m/z: Calcd for $\mathrm{C}_{17} \mathrm{H}_{27} \mathrm{~N}_{2} \mathrm{O}_{2} \mathrm{Si}[\mathrm{M}-\mathrm{H}]^{-}$319.1836, found 319.1836.

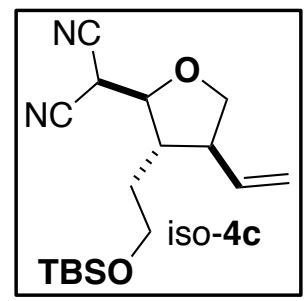

Synthesized according to Procedure C-1 (25 mg, $66 \% *, 20: 1$ d.r.).

* Note: product iso-4c was isolated with $15 \%$ Knoevenagel adduct starting material, inseparable.

Solvent system for column chromatography: Hex/EtOAc $10 \%$. 
${ }^{1}$ H NMR (400 MHz, Chloroform- $d$ ) $\delta 5.65(\mathrm{ddd}, J=17.0,10.1,8.6 \mathrm{~Hz}, 1 \mathrm{H}), 5.21-5.13(\mathrm{~m}, 2 \mathrm{H}), 4.65(\mathrm{~d}$, $J=2.2 \mathrm{~Hz}, 1 \mathrm{H}), 4.17(\mathrm{dd}, J=8.4,2.2 \mathrm{~Hz}, 1 \mathrm{H}), 4.08(\mathrm{dd}, J=8.6,7.7 \mathrm{~Hz}, 1 \mathrm{H}), 3.84-3.77(\mathrm{~m}, 2 \mathrm{H}), 3.62$ (ddd, $J=11.0,9.5,2.9 \mathrm{~Hz}, 1 \mathrm{H}), 2.67-2.58(\mathrm{~m}, 1 \mathrm{H}), 2.11-2.00(\mathrm{~m}, 1 \mathrm{H}), 1.90$ (ddt, $J=14.6,5.0,3.2 \mathrm{~Hz}$, $1 \mathrm{H}), 1.58-1.49(\mathrm{~m}, 1 \mathrm{H}), 0.90(\mathrm{~s}, 9 \mathrm{H}), 0.09(\mathrm{~s}, 6 \mathrm{H})$.

${ }^{13}$ C NMR $\left(101 \mathrm{MHz}, \mathrm{CDCl}_{3}\right) \delta 135.19,118.98,112.36,111.85,82.78,73.12,62.53,52.03,48.41,33.70$, $28.52,26.06,18.47,-5.22,-5.31$.

HRMS (ESI - TOF) m/z: Calcd for $\mathrm{C}_{17} \mathrm{H}_{27} \mathrm{~N}_{2} \mathrm{O}_{2} \mathrm{Si}[\mathrm{M}-\mathrm{H}]^{-}$319.1836, found 319.1836.

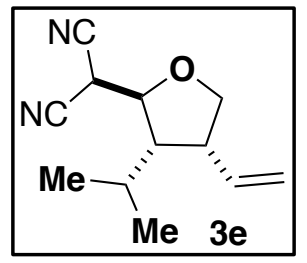

Synthesized according to Procedure C-1 (4.2 mg, $63 \%$ after 2 reaction cycle, 9:1 d.r.).

Solvent system for column chromatography: Hex/EtOAc $20 \%$.

${ }^{1}$ H NMR (400 MHz, Chloroform-d) $\delta 5.80$ (dt, $\left.J=17.3,9.8 \mathrm{~Hz}, 1 \mathrm{H}\right), 5.22-5.18(\mathrm{~m}, 1 \mathrm{H}), 5.17(\mathrm{~s}, 1 \mathrm{H})$, $4.23(\mathrm{dd}, J=7.4,2.6 \mathrm{~Hz}, 1 \mathrm{H}), 4.19(\mathrm{dd}, J=8.6,5.3 \mathrm{~Hz}, 1 \mathrm{H}), 3.87(\mathrm{~d}, J=2.9 \mathrm{~Hz}, 1 \mathrm{H}), 3.85(\mathrm{~d}, J=2.5 \mathrm{~Hz}$, $1 \mathrm{H}), 3.13-3.01(\mathrm{~m}, 1 \mathrm{H}), 2.10(\mathrm{dt}, J=9.0,7.1 \mathrm{~Hz}, 1 \mathrm{H}), 1.81(\mathrm{dp}, J=9.0,6.7 \mathrm{~Hz}, 1 \mathrm{H}), 1.00(\mathrm{~d}, J=6.6 \mathrm{~Hz}$, $3 \mathrm{H}), 0.95(\mathrm{~d}, J=6.7 \mathrm{~Hz}, 3 \mathrm{H})$.

${ }^{13} \mathbf{C}$ NMR $\left(101 \mathrm{MHz}, \mathrm{CDCl}_{3}\right) \delta 134.74,118.03,111.90,111.39,79.63,74.75,53.77,47.41,30.28,27.30$, 21.88, 21.28 .

HRMS (ESI - TOF) m/z: Calcd for $\mathrm{C}_{12} \mathrm{H}_{15} \mathrm{~N}_{2} \mathrm{O}[\mathrm{M}-\mathrm{H}]^{-}$203.1179, found 203.1179.

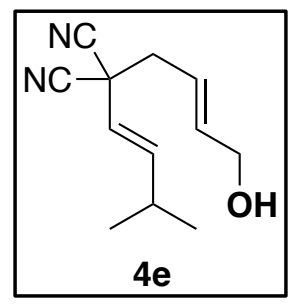

Deprotected $E$-1e recovered after Procedure C-1 on 3e.

${ }^{1}$ H NMR (400 MHz, Chloroform- $d$ ) $\delta 6.17(\mathrm{dd}, J=15.3,6.7 \mathrm{~Hz}, 1 \mathrm{H}), 5.99(\mathrm{dtt}, J=15.3,5.0,1.1 \mathrm{~Hz}, 1 \mathrm{H})$, $5.77(\mathrm{dtt}, J=15.1,7.3,1.7 \mathrm{~Hz}, 1 \mathrm{H}), 5.34(\mathrm{dd}, J=15.3,1.4 \mathrm{~Hz}, 1 \mathrm{H}), 4.22$ (ddt, $J=5.1,1.8,0.9 \mathrm{~Hz}, 2 \mathrm{H})$, $2.73(\mathrm{dq}, J=7.4,1.1 \mathrm{~Hz}, 2 \mathrm{H}), 2.43(\mathrm{dqd}, J=13.5,6.8,1.5 \mathrm{~Hz}, 1 \mathrm{H}), 1.06(\mathrm{~d}, J=6.8 \mathrm{~Hz}, 6 \mathrm{H})$.

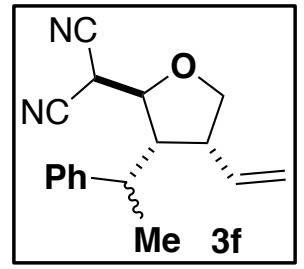

Synthesized according to Procedure C-1 (7.5 mg, 34 \%, 1:1 d.r., separable diastereomers).

Solvent system for column chromatography: Hex/EtOAc $20 \%$.

Diastereomer 1:

${ }^{1}$ H NMR (400 MHz, Chloroform- $d$ ) $\delta 7.42-7.36(\mathrm{~m}, 2 \mathrm{H}), 7.35-7.30(\mathrm{~m}, 1 \mathrm{H}), 7.22-7.18(\mathrm{~m}, 2 \mathrm{H}), 5.92$ $(\mathrm{dt}, J=17.4,9.9 \mathrm{~Hz}, 1 \mathrm{H}), 5.32(\mathrm{dd}, J=3.5,1.3 \mathrm{~Hz}, 1 \mathrm{H}), 5.29(\mathrm{~d}, J=1.2 \mathrm{~Hz}, 1 \mathrm{H}), 4.17(\mathrm{dd}, J=8.7,4.4 \mathrm{~Hz}$, $1 \mathrm{H}), 3.95(\mathrm{~d}, J=2.4 \mathrm{~Hz}, 1 \mathrm{H}), 3.93(\mathrm{~d}, J=1.9 \mathrm{~Hz}, 1 \mathrm{H}), 3.10(\mathrm{ddd}, J=10.1,6.0,4.2 \mathrm{~Hz}, 1 \mathrm{H}), 2.68(\mathrm{dq}, J=$ 11.2, $6.7 \mathrm{~Hz}, 1 \mathrm{H}), 2.44$ (ddd, $J=11.3,9.4,6.1 \mathrm{~Hz}, 1 \mathrm{H}), 1.84(\mathrm{~d}, J=1.9 \mathrm{~Hz}, 1 \mathrm{H}), 1.37$ (d, $J=6.7 \mathrm{~Hz}, 3 \mathrm{H})$. 
${ }^{13}$ C NMR $\left(101 \mathrm{MHz}, \mathrm{CDCl}_{3}\right) \delta 144.00,134.62,129.83,128.24,126.93,118.44,111.88,111.24,80.11$, 74.62, 53.88, 47.07, 39.35, 28.53, 19.86 .

Diastereomer 2:

${ }^{1}$ H NMR (400 MHz, Chloroform- $d$ ) $\delta 7.36-7.29$ (m, 2H), $7.26-7.21(\mathrm{~m}, 1 \mathrm{H}), 7.20-7.16(\mathrm{~m}, 2 \mathrm{H}), 5.79$ (ddd, $J=17.2,10.4,8.9 \mathrm{~Hz}, 1 \mathrm{H}), 5.14$ (dd, $J=10.3,1.3 \mathrm{~Hz}, 1 \mathrm{H}), 4.88$ (dt, $J=17.2,1.2 \mathrm{~Hz}, 1 \mathrm{H}), 4.33$ (dd, $J=7.4,2.5 \mathrm{~Hz}, 1 \mathrm{H}), 4.19$ (dd, $J=8.7,5.2 \mathrm{~Hz}, 1 \mathrm{H}), 3.86$ (dd, $J=8.7,3.1 \mathrm{~Hz}, 1 \mathrm{H}), 3.80$ (d, $J=2.5 \mathrm{~Hz}, 1 \mathrm{H})$, $2.96(\mathrm{dq}, J=9.7,7.0 \mathrm{~Hz}, 1 \mathrm{H}), 2.88-2.79(\mathrm{~m}, 1 \mathrm{H}), 2.72(\mathrm{dt}, J=9.4,7.1 \mathrm{~Hz}, 1 \mathrm{H}), 1.26(\mathrm{~d}, J=7.0 \mathrm{~Hz}, 3 \mathrm{H})$. ${ }^{13}$ C NMR $\left(101 \mathrm{MHz}, \mathrm{CDCl}_{3}\right) \delta 144.29,134.19,128.91,127.29,127.05,118.67,111.75,111.23,79.43$, 74.52, 52.69, 47.12, 38.70, 30.43, 21.15 .

HRMS (ESI - TOF) m/z: Calcd for $\mathrm{C}_{17} \mathrm{H}_{17} \mathrm{~N}_{2} \mathrm{O}[\mathrm{M}-\mathrm{H}]^{-}$265.1335, found 265.1339.

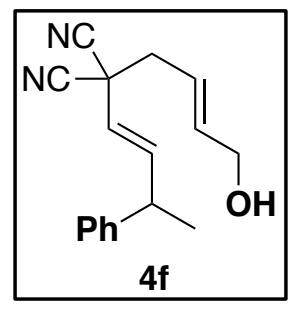

Deprotected $E$-1f recovered after Procedure C-1 on 3f.

${ }^{1}$ H NMR (400 MHz, Chloroform- $d$ ) $\delta 7.38-7.31(\mathrm{~m}, 2 \mathrm{H}), 7.28-7.25(\mathrm{~m}, 1 \mathrm{H}), 7.20-7.15(\mathrm{~m}, 2 \mathrm{H}), 6.37$ $(\mathrm{dd}, J=15.3,6.6 \mathrm{~Hz}, 1 \mathrm{H}), 5.95(\mathrm{dtt}, J=15.4,5.0,1.2 \mathrm{~Hz}, 1 \mathrm{H}), 5.72(\mathrm{dtt}, J=15.1,7.4,1.7 \mathrm{~Hz}, 1 \mathrm{H}), 5.36$ $(\mathrm{dd}, J=15.3,1.5 \mathrm{~Hz}, 1 \mathrm{H}), 4.17(\mathrm{dd}, J=5.0,1.6 \mathrm{~Hz}, 2 \mathrm{H}), 3.59(\mathrm{pd}, J=7.0,1.5 \mathrm{~Hz}, 1 \mathrm{H}), 2.74(\mathrm{dt}, J=7.4$, $1.0 \mathrm{~Hz}, 2 \mathrm{H}), 1.43(\mathrm{~d}, J=7.1 \mathrm{~Hz}, 3 \mathrm{H})$.

${ }^{13}$ C NMR $\left(101 \mathrm{MHz}, \mathrm{CDCl}_{3}\right) \delta 143.28,142.79,138.34,128.95,127.30,127.07,120.78,119.78,114.23$, $114.17,62.68,42.29,41.92,39.31,20.77$.

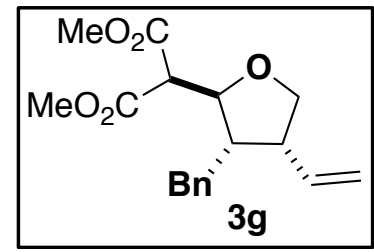

Synthesized according to Procedure C-2 (3 mg, $36 \%$ after 2 reaction cycle, 8:1 d.r.).

Solvent system for column chromatography: Hex/EtOAc $20 \%$.

${ }^{1}$ H NMR $(600 \mathrm{MHz}$, Chloroform- $d) \delta 7.28(\mathrm{t}, J=7.6 \mathrm{~Hz}, 3 \mathrm{H}), 7.21-7.18(\mathrm{~m}, 1 \mathrm{H}), 7.16(\mathrm{~d}, J=6.6 \mathrm{~Hz}$, 2H), 5.87 (ddd, $J=17.1,10.3,8.8 \mathrm{~Hz}, 1 \mathrm{H}), 5.17$ (dd, $J=10.4,1.7 \mathrm{~Hz}, 1 \mathrm{H}), 5.09$ (dt, $J=17.1,1.2 \mathrm{~Hz}, 1 \mathrm{H}$ ), 4.39 (dd, $J=7.2,5.8 \mathrm{~Hz}, 1 \mathrm{H}), 3.98$ (dd, $J=8.5,6.2 \mathrm{~Hz}, 1 \mathrm{H}), 3.79-3.76(\mathrm{~m}, 1 \mathrm{H}), 3.72(\mathrm{~s}, 3 \mathrm{H}), 3.56$ (s, $3 \mathrm{H}), 3.36(\mathrm{~d}, J=7.2 \mathrm{~Hz}, 1 \mathrm{H}), 2.94(\mathrm{dq}, J=8.5,6.2 \mathrm{~Hz}, 1 \mathrm{H}), 2.79(\mathrm{dd}, J=13.7,7.0 \mathrm{~Hz}, 1 \mathrm{H}), 2.63-2.56$ (m, 1H), 2.50 (dd, $J=13.7,8.2 \mathrm{~Hz}, 1 \mathrm{H})$.

${ }^{13} \mathbf{C}$ NMR $\left(151 \mathrm{MHz}, \mathrm{CDCl}_{3}\right) \delta 167.73,167.53,139.92,135.37,129.11,128.60,126.36,117.88,80.75$, 72.33, 56.70, 52.69, 52.66, 47.97, 46.48, 34.43.

HRMS (ESI - TOF) m/z: Calcd for $\mathrm{C}_{18} \mathrm{H}_{22} \mathrm{NaO}_{5}[\mathrm{M}+\mathrm{Na}]^{+} 341.1559$, found 341.1351.

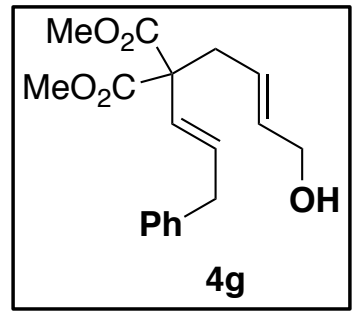


Deprotected $E-\mathbf{1 g}$ recovered after Procedure C-1 on 3g.

${ }^{1}$ H NMR (600 MHz, Chloroform- $d$ ) $\delta 7.29(\mathrm{t}, J=7.6 \mathrm{~Hz}, 2 \mathrm{H}), 7.22-7.18(\mathrm{~m}, 1 \mathrm{H}), 7.17-7.13(\mathrm{~m}, 2 \mathrm{H})$, $5.99(\mathrm{dt}, J=16.0,1.5 \mathrm{~Hz}, 1 \mathrm{H}), 5.73(\mathrm{dt}, J=16.0,7.0 \mathrm{~Hz}, 1 \mathrm{H}), 5.67$ (dtt, $J=15.4,5.7,1.3 \mathrm{~Hz}, 1 \mathrm{H}), 5.54$ $(\mathrm{dtt}, J=15.6,7.2,1.4 \mathrm{~Hz}, 1 \mathrm{H}), 4.02(\mathrm{dd}, J=5.7,1.4 \mathrm{~Hz}, 2 \mathrm{H}), 3.73(\mathrm{~s}, 6 \mathrm{H}), 3.43(\mathrm{~d}, J=7.0 \mathrm{~Hz}, 2 \mathrm{H}), 2.80$ (dd, $J=7.3,1.3 \mathrm{~Hz}, 2 \mathrm{H}$ ).

${ }^{13}$ C NMR $\left(151 \mathrm{MHz}, \mathrm{CDCl}_{3}\right) \delta 170.79,139.76,133.76,132.11,128.64,128.60,127.65,126.39,126.28$, $63.45,59.62,52.84,39.16,38.36$.

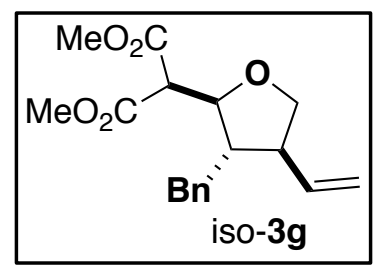

Synthesized according to Procedure C-2 (1.5 mg, $39 \%$ after 2 reaction cycle, $12: 1$ d.r.).

Solvent system for column chromatography: Hex/EtOAc $20 \%$.

${ }^{1}$ H NMR (400 MHz, Chloroform- $d$ ) $\delta 7.30-7.26(\mathrm{~m}, 2 \mathrm{H}), 7.22-7.18(\mathrm{~m}, 1 \mathrm{H}), 7.17-7.12(\mathrm{~m}, 2 \mathrm{H}), 5.59$ (ddd, $J=17.8,9.9,8.2 \mathrm{~Hz}, 1 \mathrm{H}), 5.03-4.97(\mathrm{~m}, 1 \mathrm{H}), 4.96(\mathrm{t}, J=1.1 \mathrm{~Hz}, 1 \mathrm{H}), 4.36(\mathrm{t}, J=6.7 \mathrm{~Hz}, 1 \mathrm{H}), 3.95$ (dd, $J=8.7,7.4 \mathrm{~Hz}, 1 \mathrm{H}), 3.73(\mathrm{~s}, 3 \mathrm{H}), 3.68(\mathrm{~s}, 3 \mathrm{H}), 3.61(\mathrm{t}, J=8.6 \mathrm{~Hz}, 1 \mathrm{H}), 3.26(\mathrm{~d}, J=6.4 \mathrm{~Hz}, 1 \mathrm{H}), 2.80$ (dd, $J=13.7,6.9 \mathrm{~Hz}, 1 \mathrm{H}), 2.72$ (dd, $J=13.8,7.1 \mathrm{~Hz}, 1 \mathrm{H}), 2.64$ (p, $J=8.1 \mathrm{~Hz}, 1 \mathrm{H}), 2.37-2.26(\mathrm{~m}, 1 \mathrm{H})$. ${ }^{13}$ C NMR $\left(101 \mathrm{MHz}, \mathrm{CDCl}_{3}\right) \delta 167.90,167.74,139.12,137.44,129.33,128.61,126.58,116.82,82.06$, $72.21,56.19,52.74,52.62,50.21,50.14,38.37$.

HRMS (ESI - TOF) m/z: Calcd for $\mathrm{C}_{18} \mathrm{H}_{22} \mathrm{NaO}_{5}[\mathrm{M}+\mathrm{Na}]^{+} 341.1559$, found 341.1351.

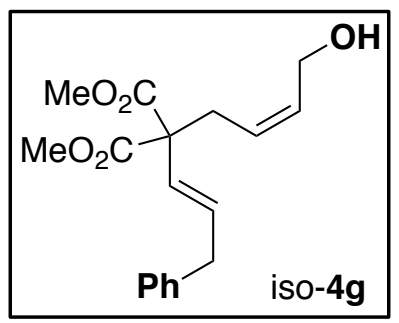

Deprotected Z-1g recovered after Procedure C-1 on iso-3g.

${ }^{1}$ H NMR $(400 \mathrm{MHz}$, Chloroform- $d$ ) $\delta 7.35-7.29(\mathrm{~m}, 2 \mathrm{H}), 7.26-7.20(\mathrm{~m}, 1 \mathrm{H}), 7.20-7.15(\mathrm{~m}, 2 \mathrm{H}), 6.03$ $(\mathrm{dt}, J=16.0,1.5 \mathrm{~Hz}, 1 \mathrm{H}), 5.81-5.70(\mathrm{~m}, 2 \mathrm{H}), 5.44(\mathrm{dtt}, J=10.6,7.7,1.4 \mathrm{~Hz}, 1 \mathrm{H}), 4.15(\mathrm{~d}, J=6.8 \mathrm{~Hz}$, $2 \mathrm{H}), 3.76(\mathrm{~s}, 6 \mathrm{H}), 3.45(\mathrm{~d}, J=6.9 \mathrm{~Hz}, 2 \mathrm{H}), 2.86(\mathrm{dd}, J=7.7,1.5 \mathrm{~Hz}, 2 \mathrm{H})$.

\section{Structural and Stereochemical assignment control experiments}

a. Conversion and diastereoselectivity ratio for Scheme 4

Below are crude NMRs for 3a, iso-3a and their respective Cope products. An overlay of the starting material (in purple) is presented against the crude mixture (in green). All peaks from each diastereomers (pink and blue) and starting material could be clearly assigned. Each red box demonstrate how conversion and diastereoselective was determined. 


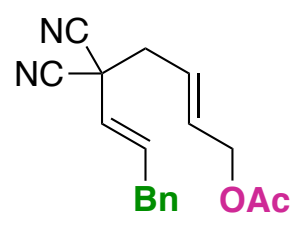

E-1a-OAc

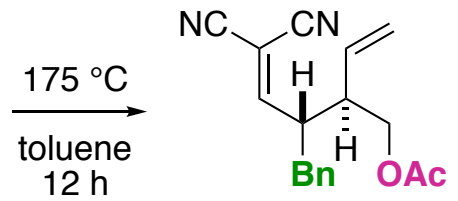

$64 \%$ conv.

$3: 1$ d.r.
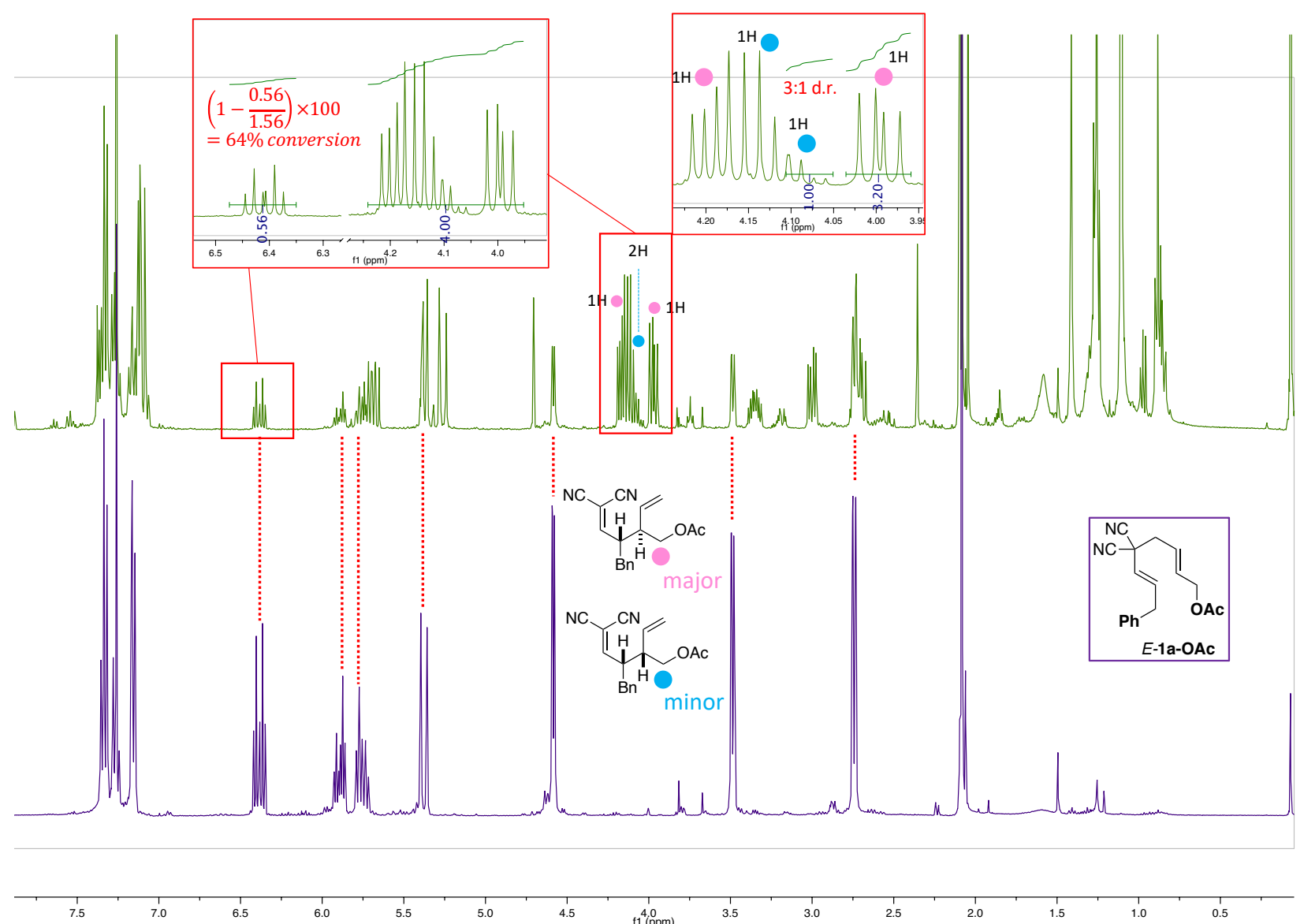


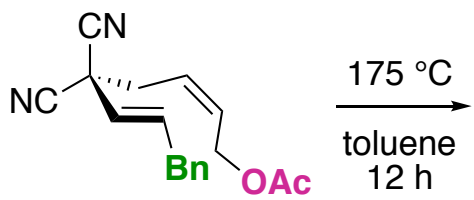

Z-1a-OAC

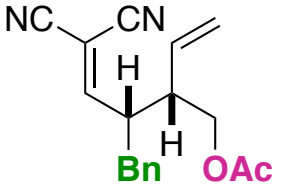

$74 \%$ conv. 5:1 d.r.
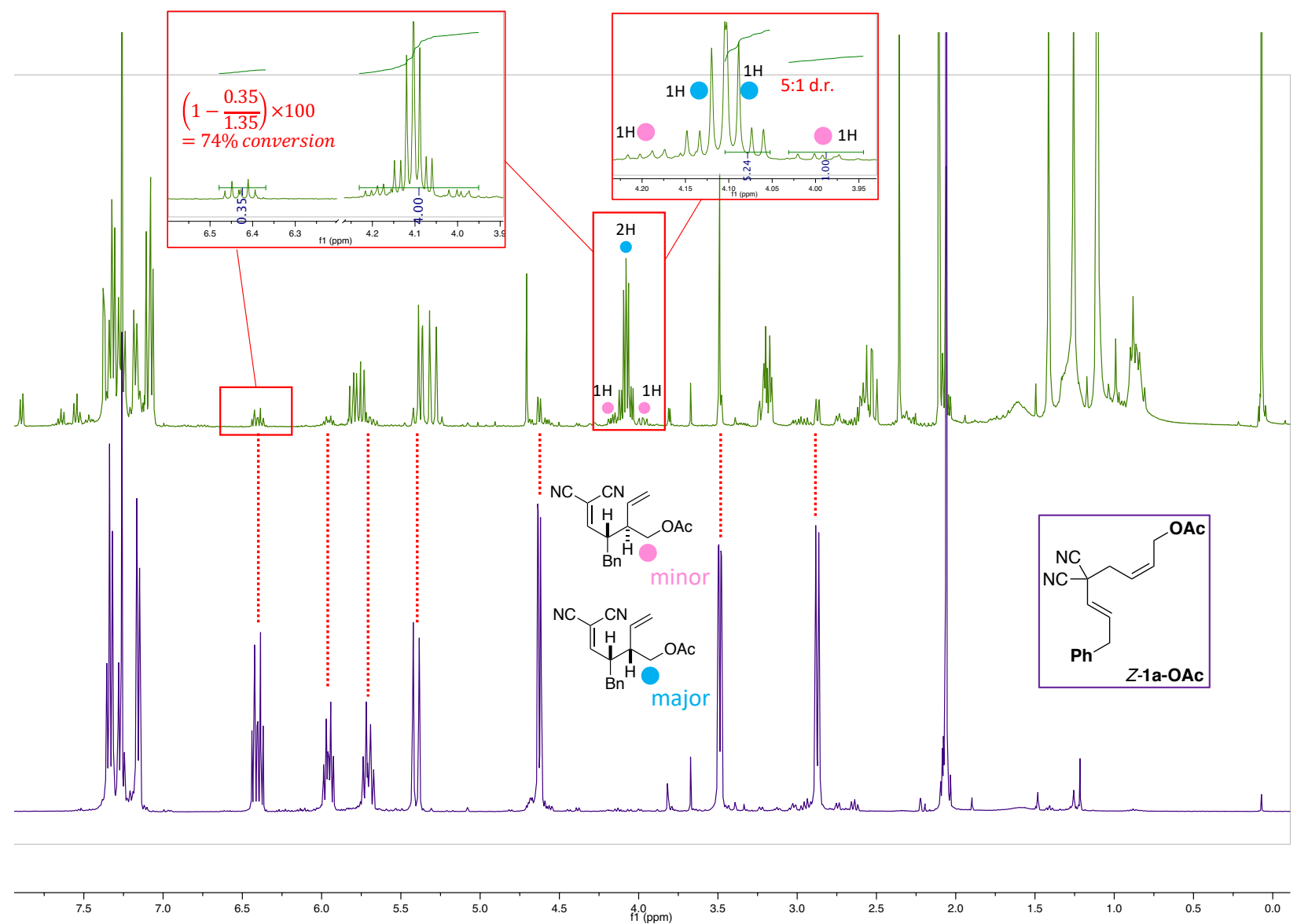

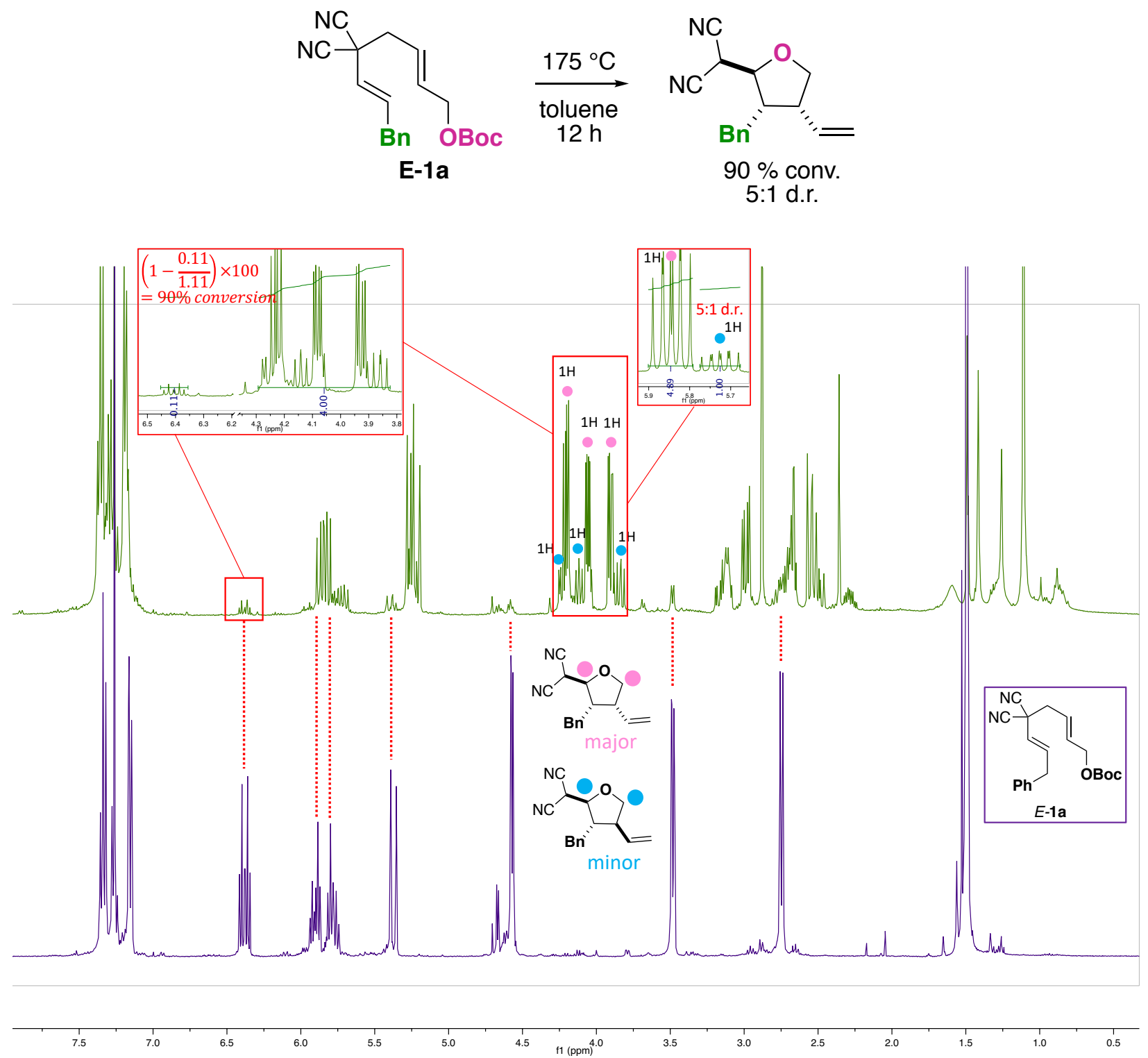


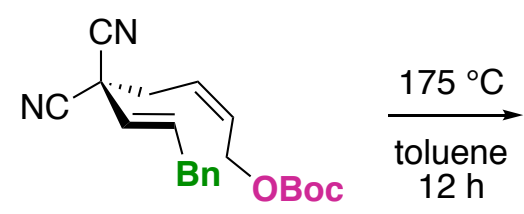

Z-1a

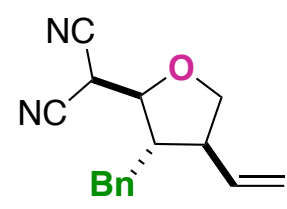

$94 \%$ conv.

$10: 1$ d.r.
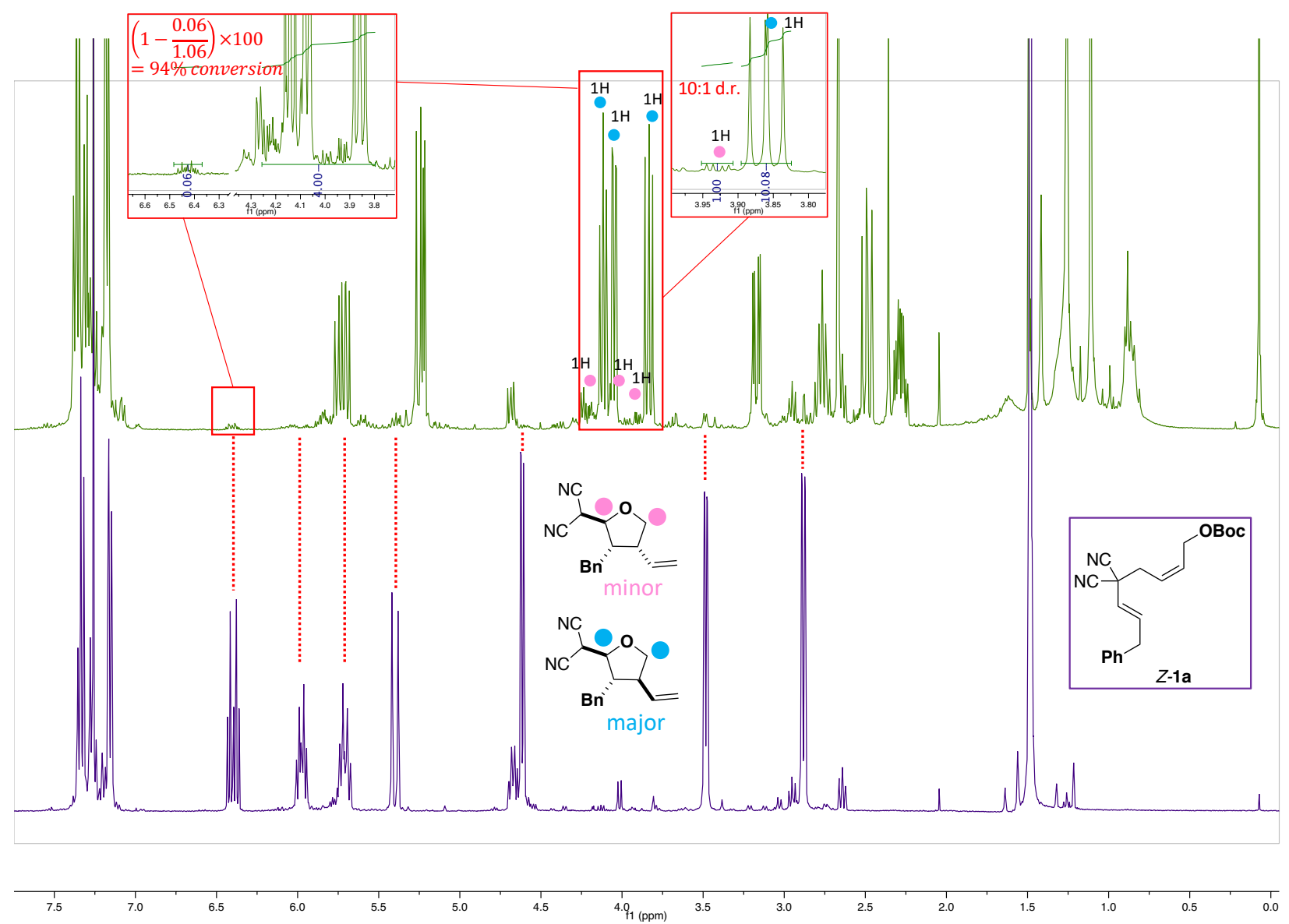

b. Stereochemical outcome of the Cope rearrangement ( 3 and 4 position)

In order to confirm the stereochemical outcome predicted by the Zimmerman-Traxler model, we performed the following experiment: $E$-1a-OAc was subjected to the Cope rearrangement conditions $\left(175^{\circ} \mathrm{C}, 12 \mathrm{~h}\right.$ in toluene). The expected Cope rearranged product $\mathbf{A}$ was observed (green pathway).

Starting from 3a (made from 1a), we performed a retro-Oxy-Michael addition as depicted in the figure below using $\mathrm{Et}_{3} \mathrm{~N}, \mathrm{Ac}_{2} \mathrm{O}$ in DMF at room temperature. The same product $\mathbf{A}$ was obtained in $60 \%$ (purple pathway). Since both reactions converge to the same product $\mathbf{A}$, we are confident about the assigned stereochemistry of 3a and iso-3a at the 3 and 4 position. (both ${ }^{1} \mathrm{H}$ NMR spectra clearly show that the same product is formed although with slightly different diastereomer ratios). 


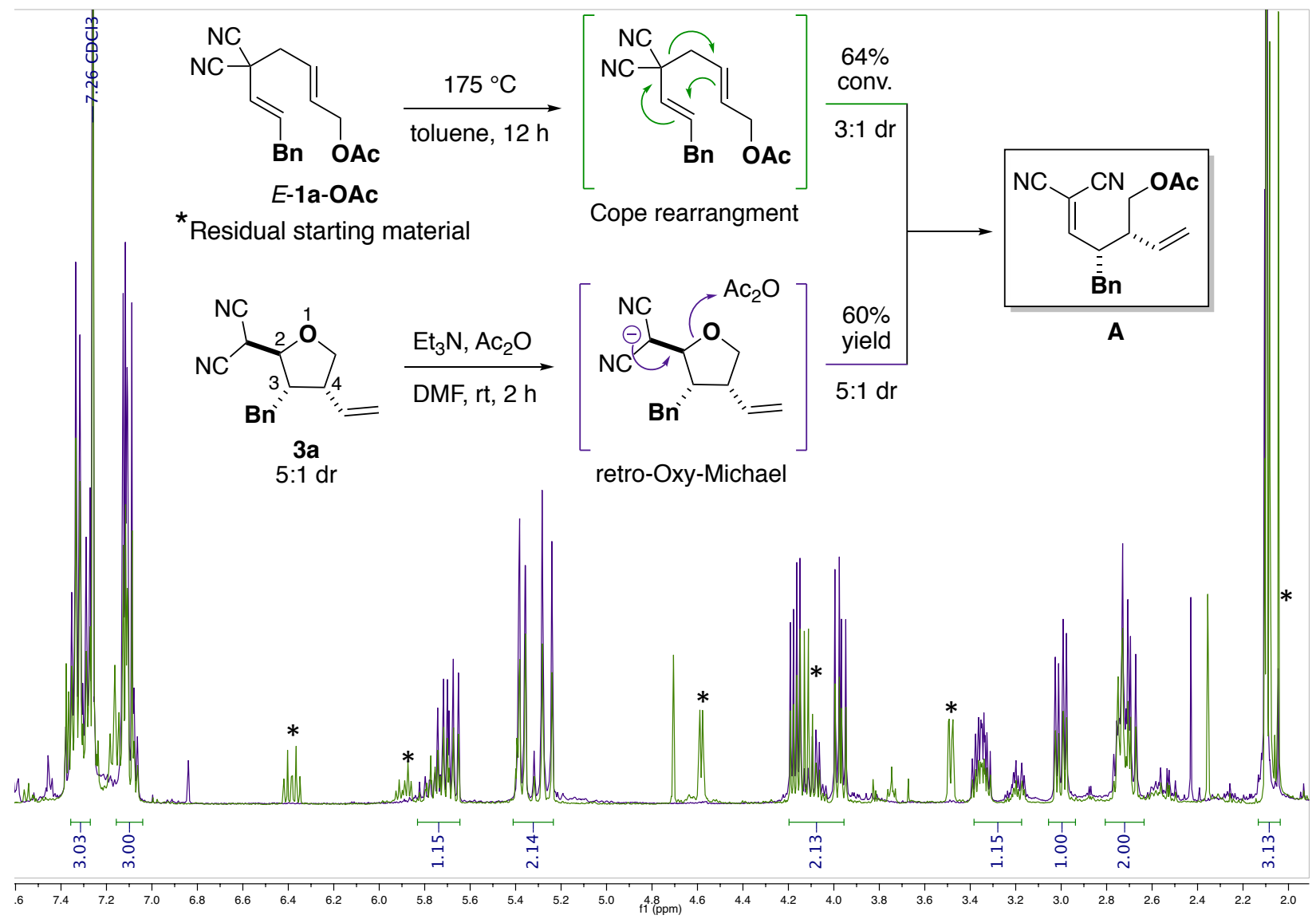

${ }^{13} \mathrm{C}$ NMR comparison of $\mathbf{3 a}$ and $\mathbf{A}$ demonstrate that they can unambiguously and easily be differentiated (please see next figure). 

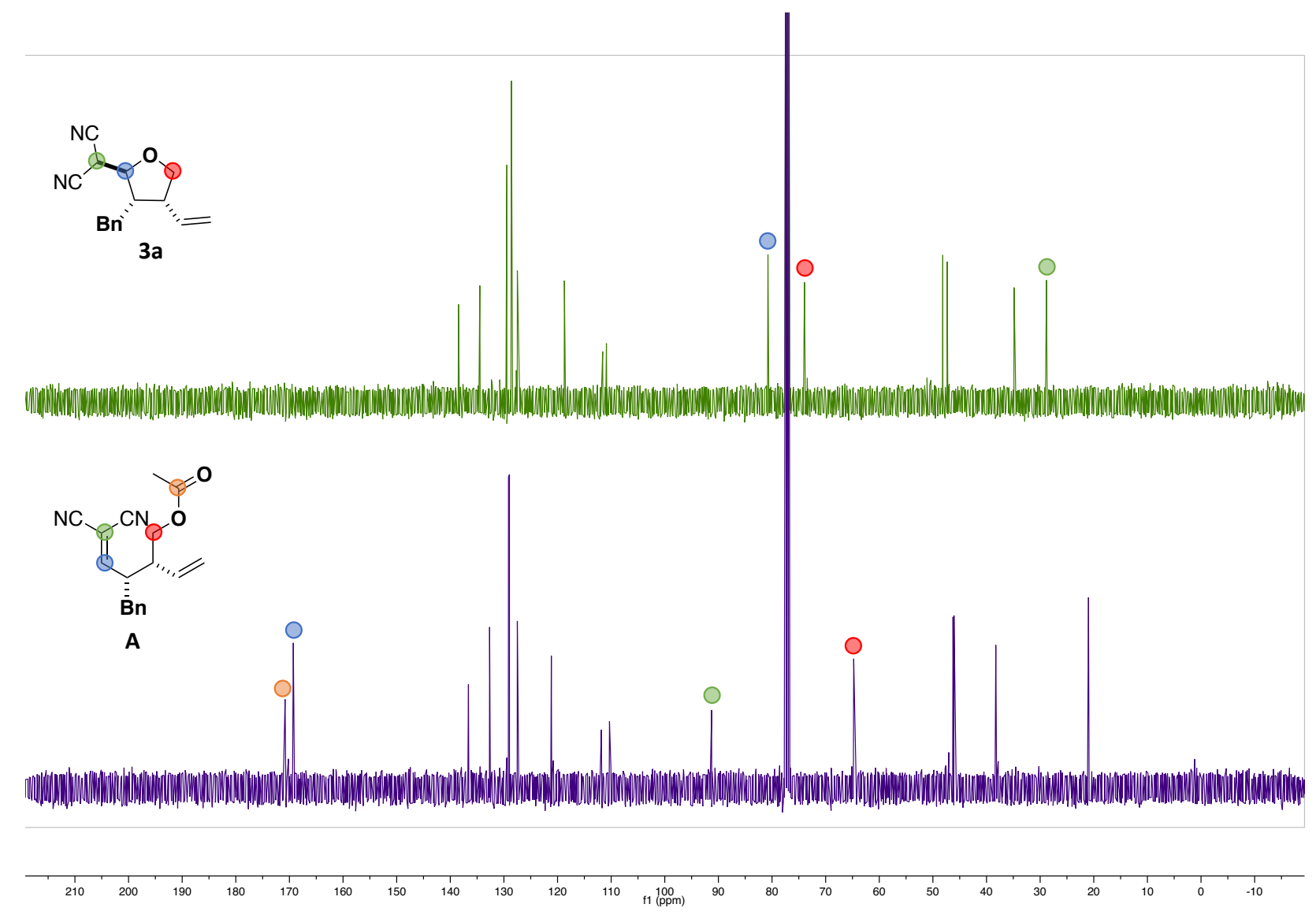

c. Stereochemical outcome of the Cope rearrangement/Oxy-Michael (2 position)

3a and iso-3a were both subjected to the same allylation conditions (described in detail in section 2.d.i.). Then, product 5a and iso-5a were subjected to ring-closing metathesis conditions (described in detail in section 2.d.ii.). While 5a gave the expected product 5b, iso-5a didn't react at all under these conditions and was entirely recovered. In this system, only one diastereomer, presenting the appropriate geometry, can react. This experiment allowed us to confirm the assigned stereochemistry of $\mathbf{3 a}$ and iso-3a at the 2 position.<smiles>C=C[C@@H]1CO[C@H](C(C#N)C#N)[C@@H]1Br</smiles><smiles>C=CC1COC(C(C#N)C#N)C1Br</smiles>
iso-3a

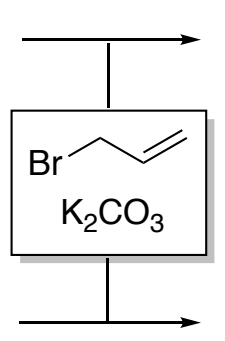

$5 a, 8: 1 d r$

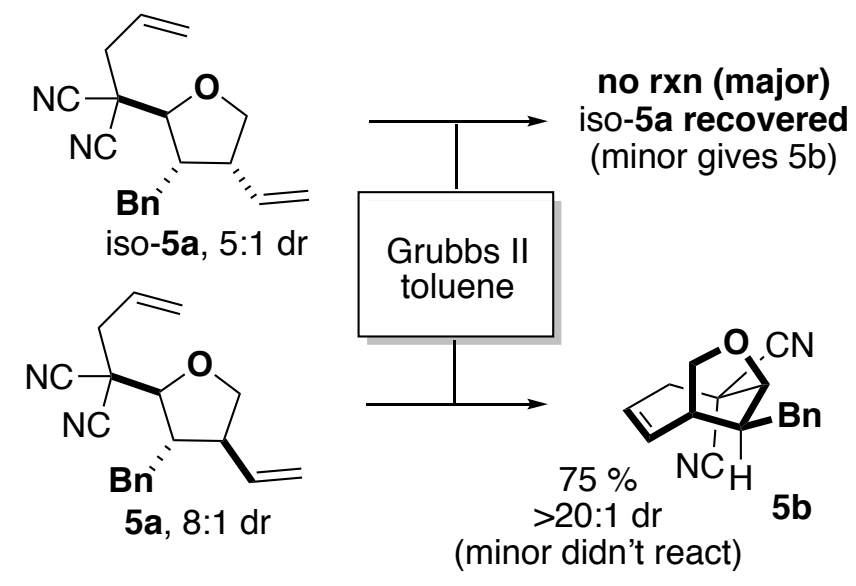

(minor didn't react) 
${ }^{1} \mathrm{H} \&{ }^{13} \mathrm{C}$ NMR

spectra 
$\frac{m}{u}$
U
0
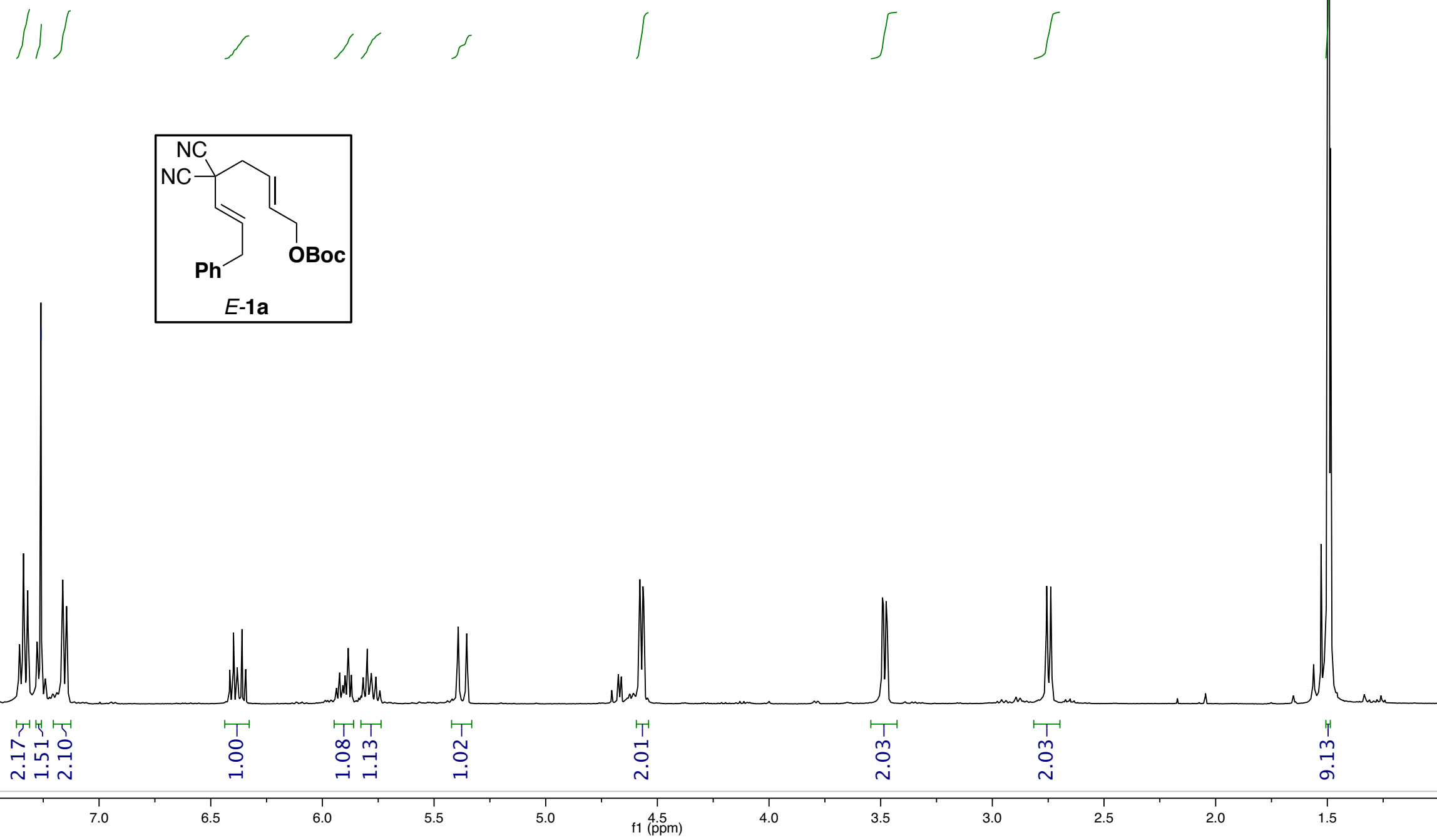


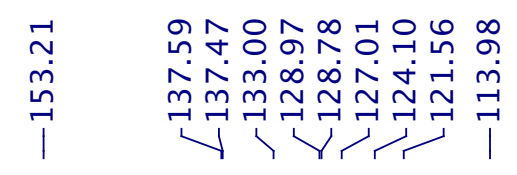

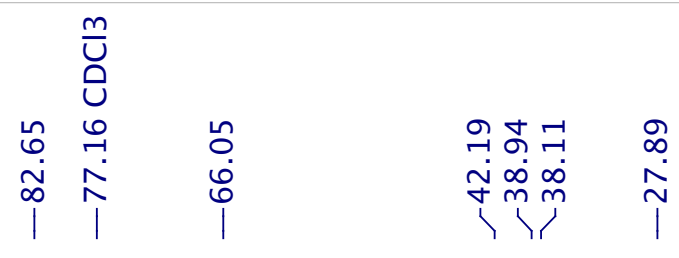

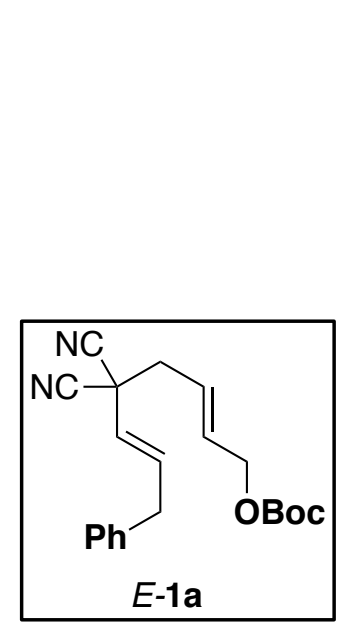

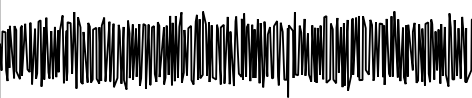

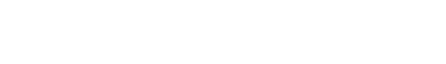




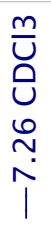

11

$\int \mathcal{S} S$

$\int$

$\int$

1

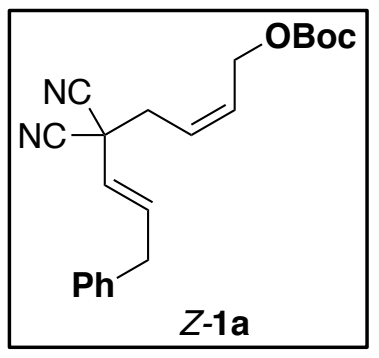

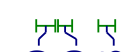

국요

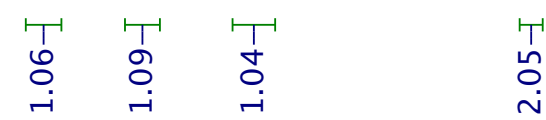

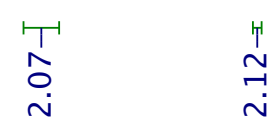

党

$T$

7.5

7.0

5.5

1.0

f1 $\begin{gathered}4.5 \\ \mathrm{ppm})\end{gathered}$

1.0

3.0

2.5

2.0

1.5 
m.

公 $\quad$ m

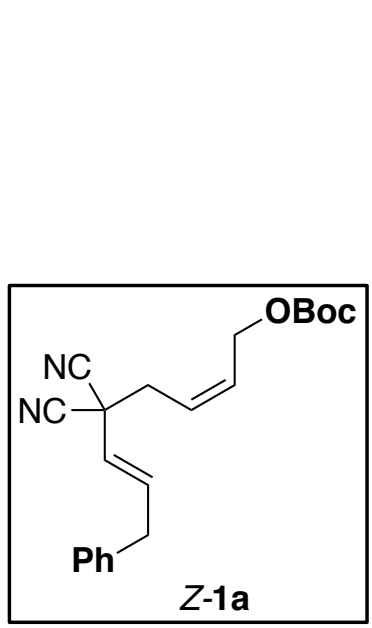

บั

$\infty$

i

함

윙교 $\stackrel{\infty}{\infty}$

$\infty \infty N$

$\underbrace{m m} \tilde{i}$

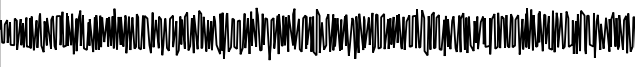

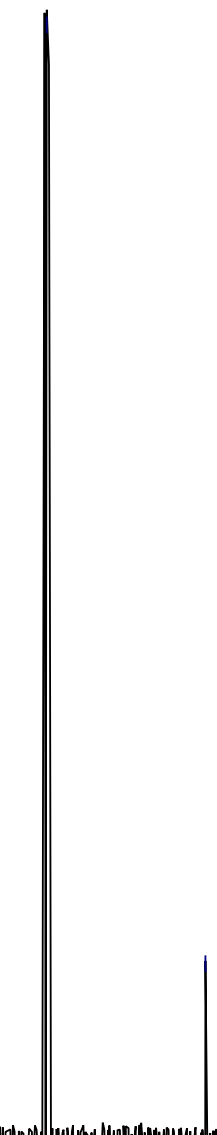

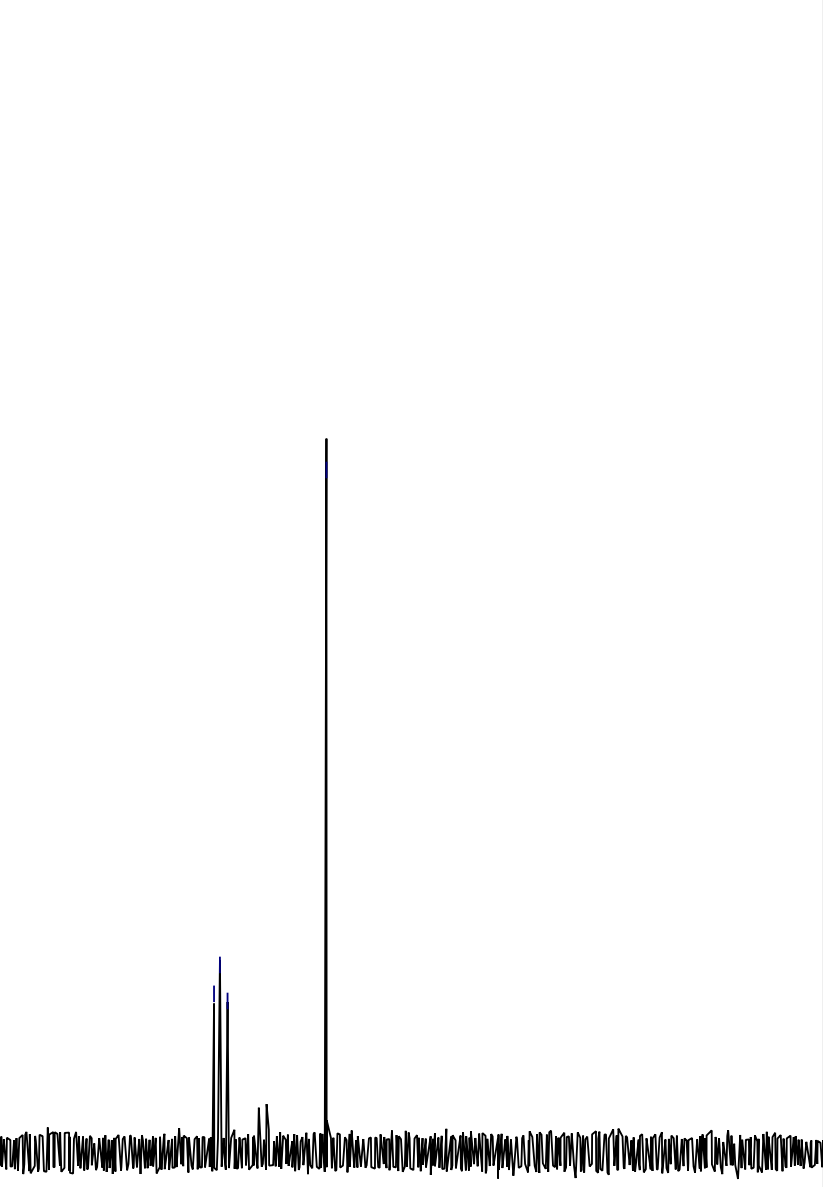

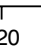


$\frac{m}{U}$
0
0
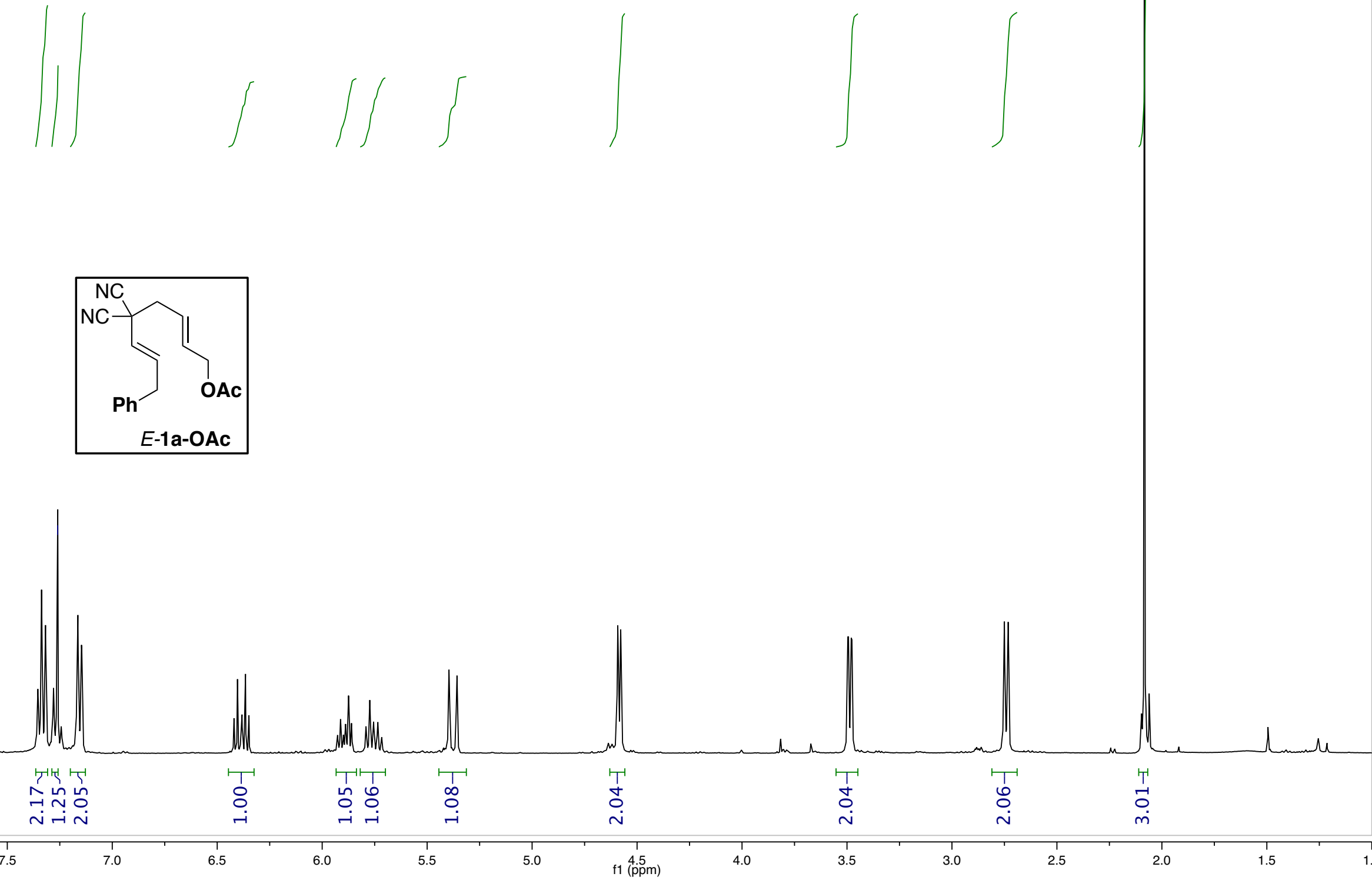


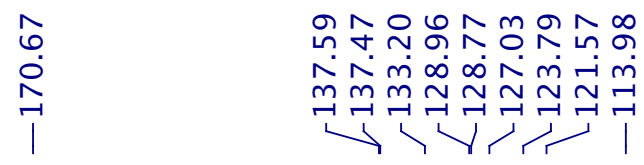

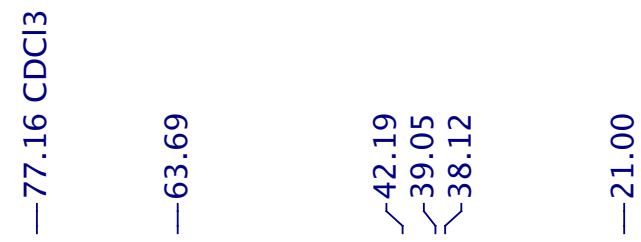

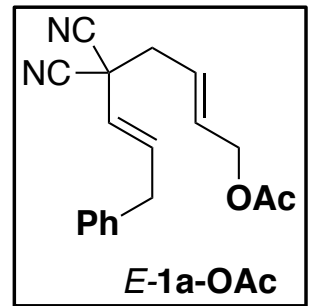

$210 \quad 200$ 
$\frac{m}{U}$
0
0

(i)
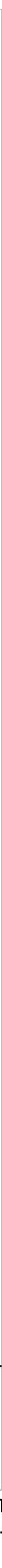


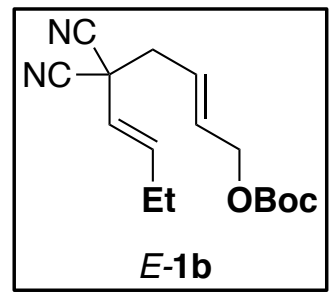


仓ั

ठ․

$\stackrel{\infty}{\sim} \stackrel{\infty}{\sim} \quad \stackrel{\infty}{\sim}$

ๆ

in

กิ

$m$
$\infty$

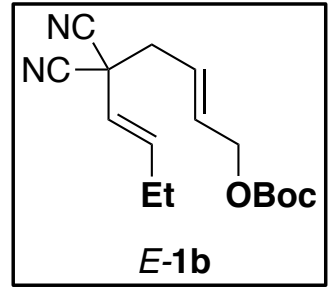

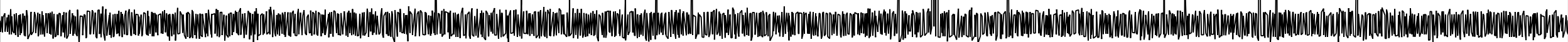


$\frac{m}{U}$
0
0
$\stackrel{0}{i}$

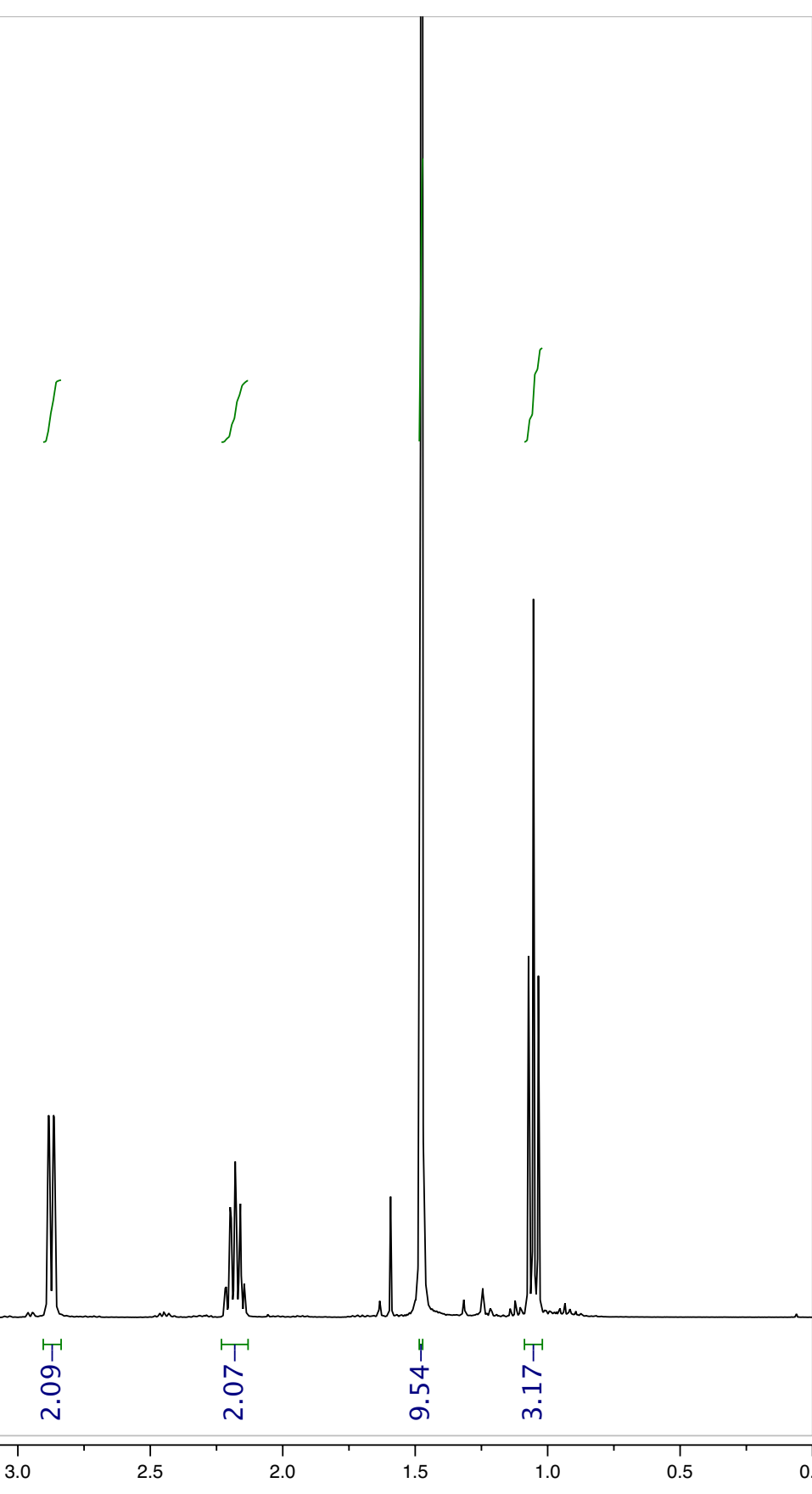




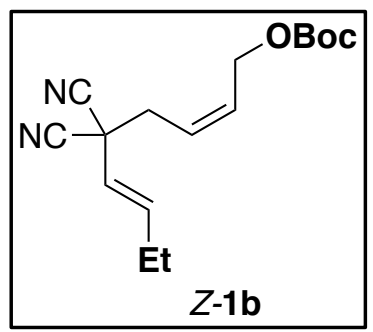

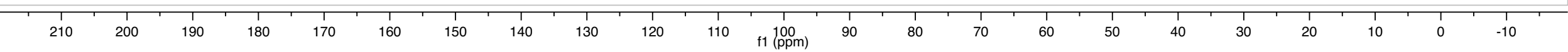



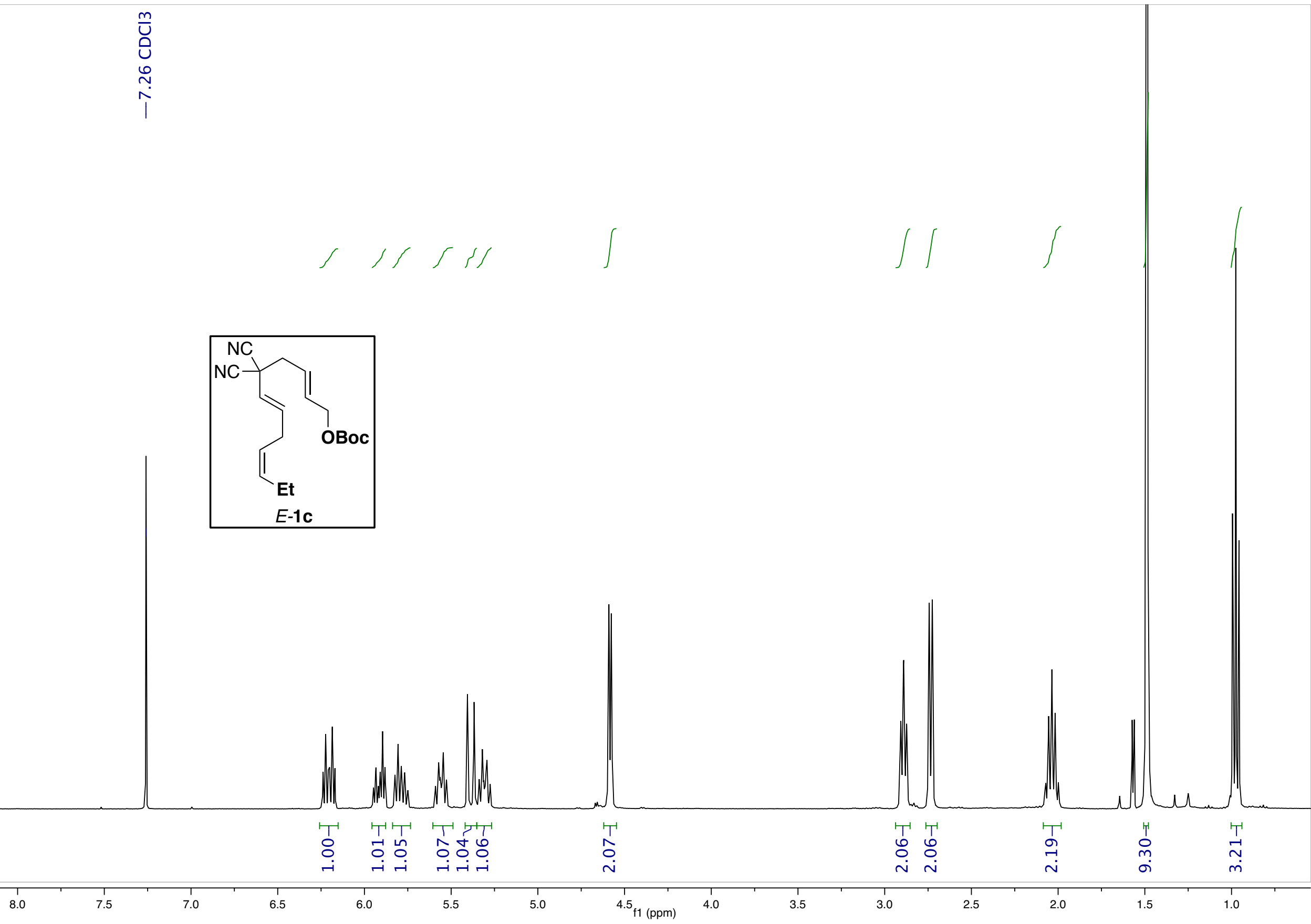


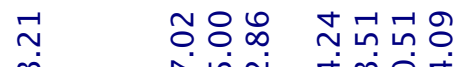

$$
\begin{aligned}
& \text { 盀 }
\end{aligned}
$$

$\begin{array}{cc} & \frac{m}{y} \\ & 0 \\ m & 0 \\ 0 & \ddots \\ i & \hat{N} \\ 0 & 1\end{array}$

$\hat{0}$
$\dot{0}$
$\dot{0}$
1

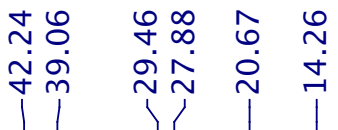

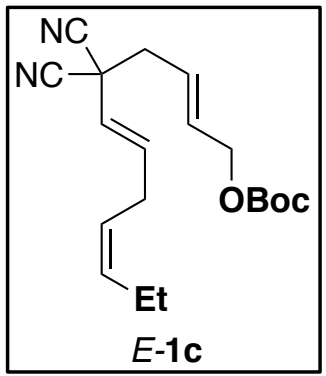




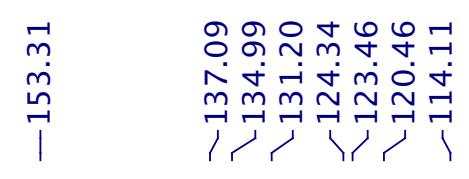

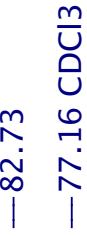

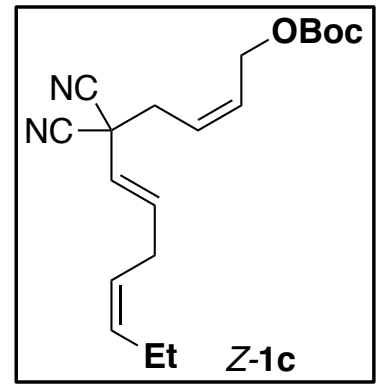




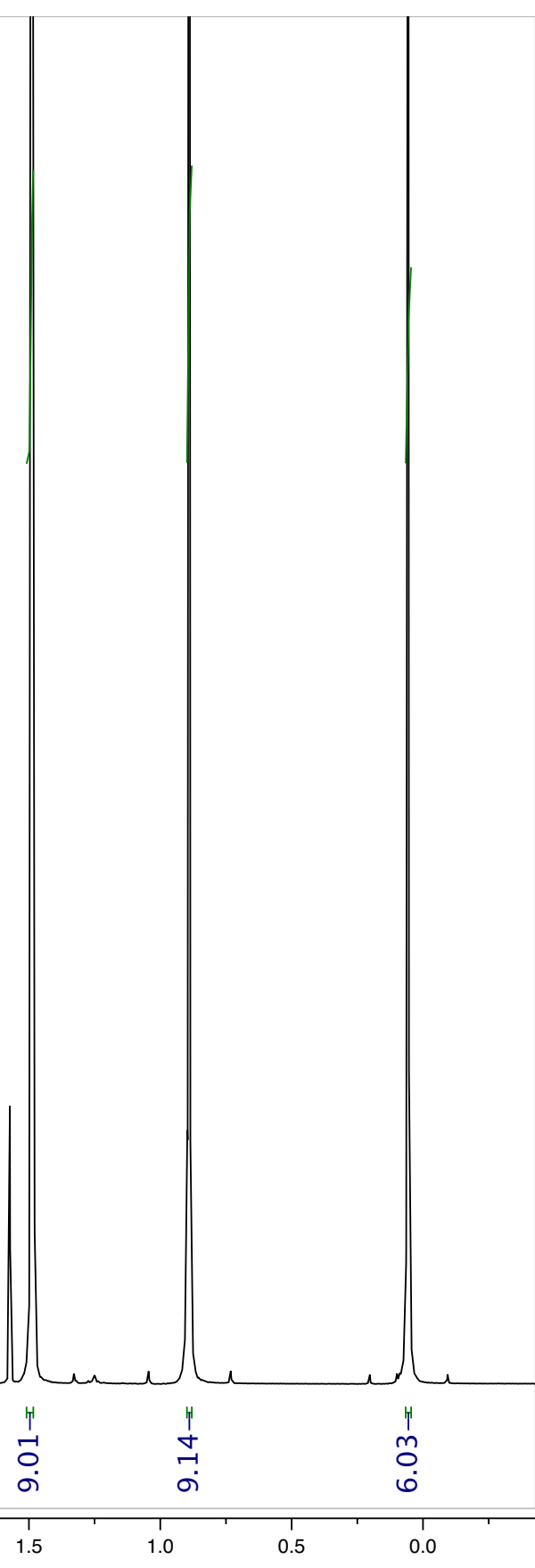




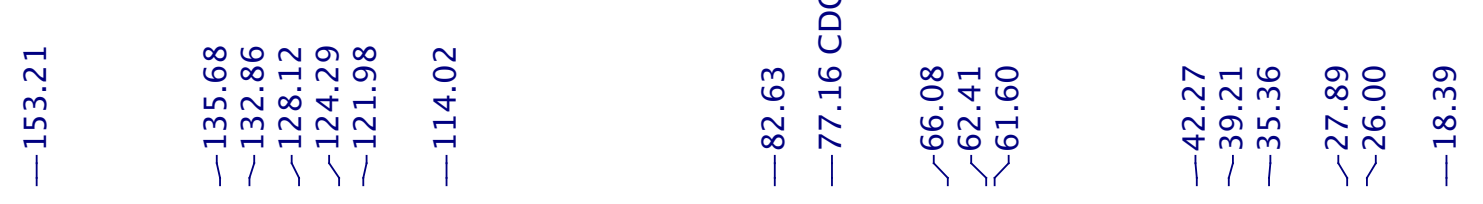
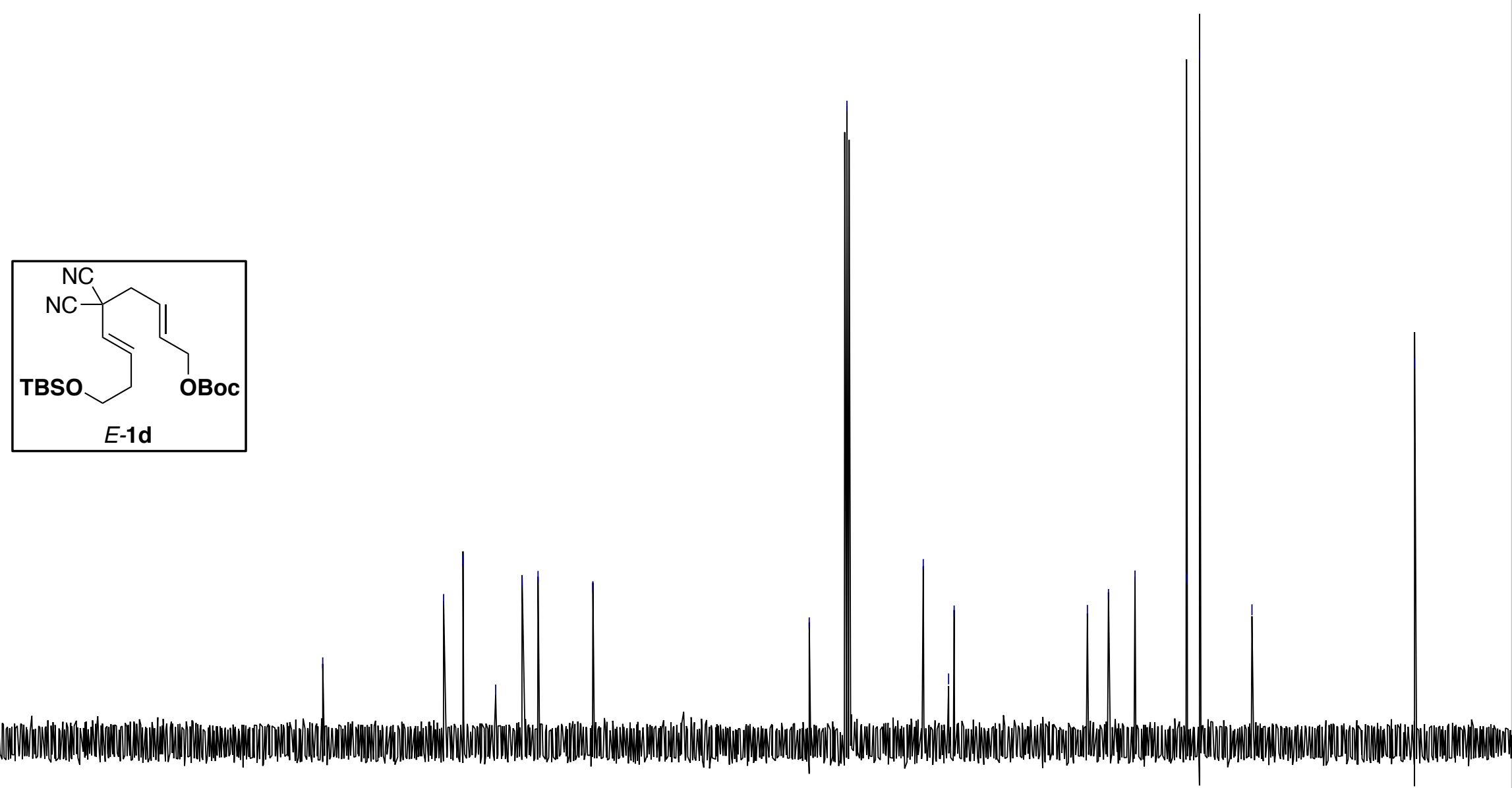


\section{* Trace of Knoevenagel starting material}

$m$
$\bar{U}$
0
0

$i$
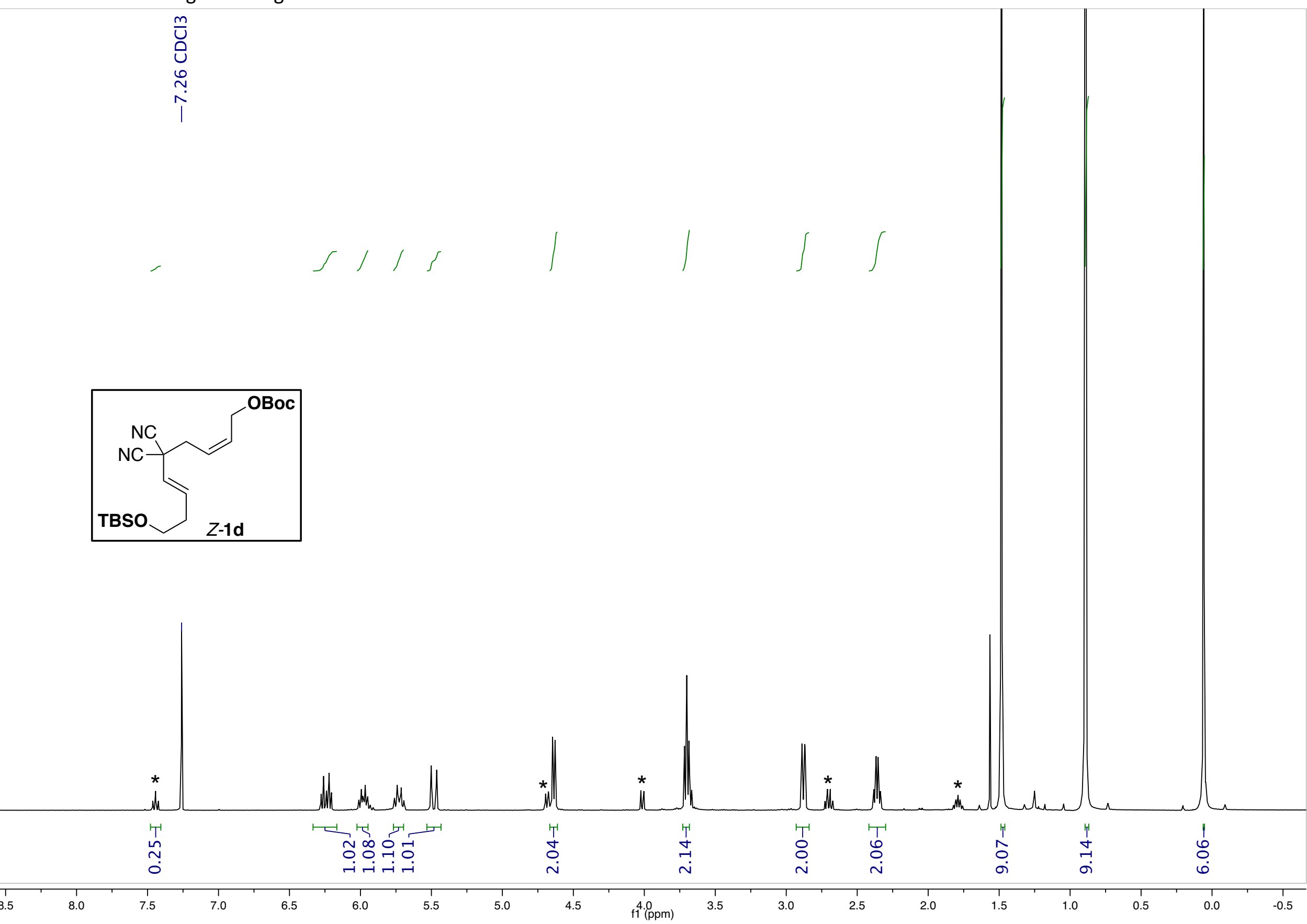


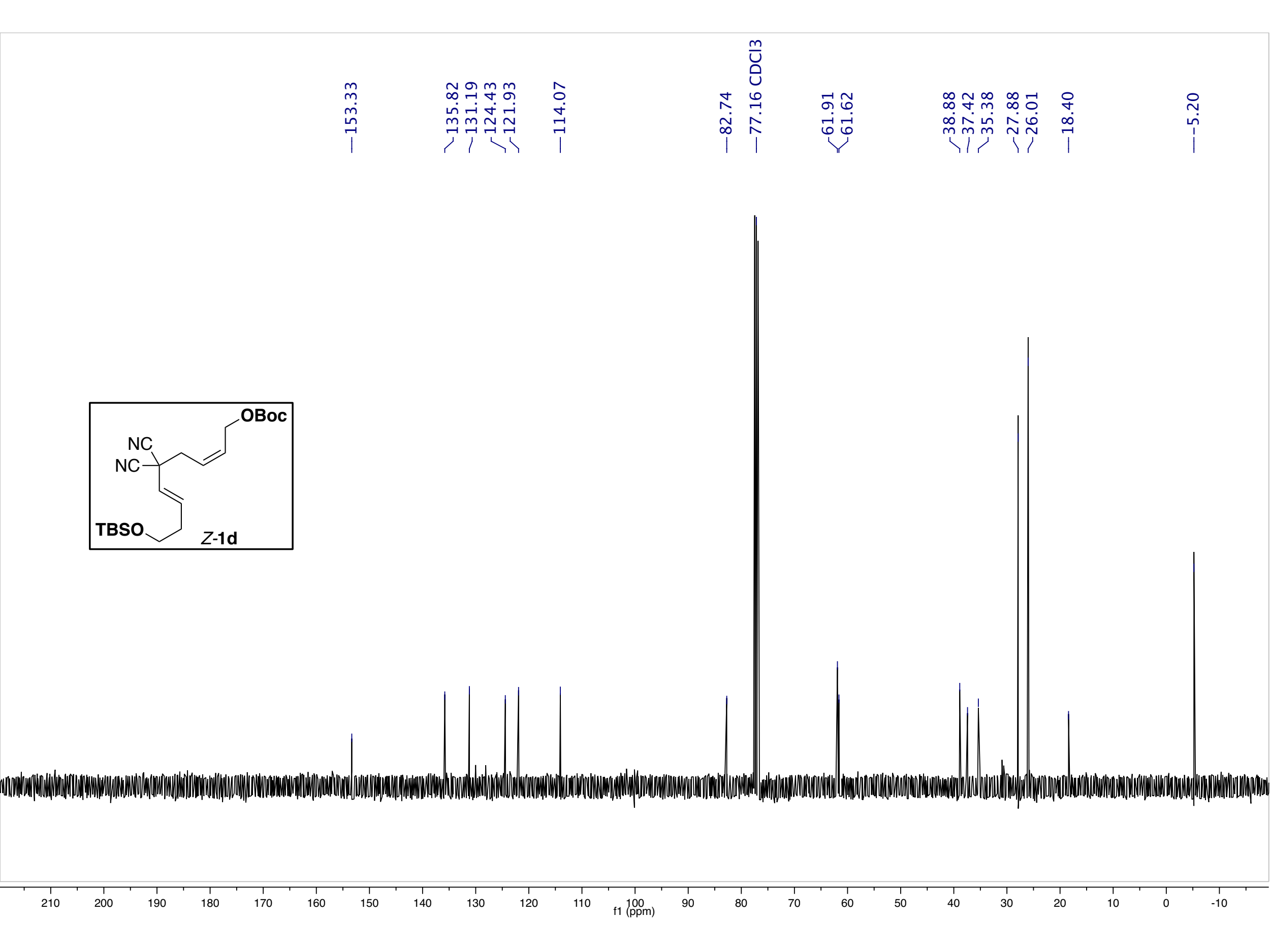




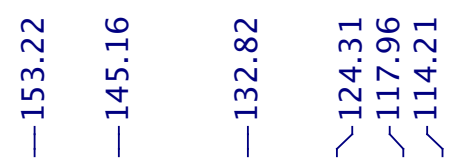

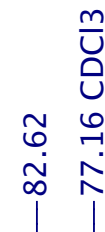

$\hat{0}$
6
0

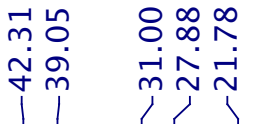
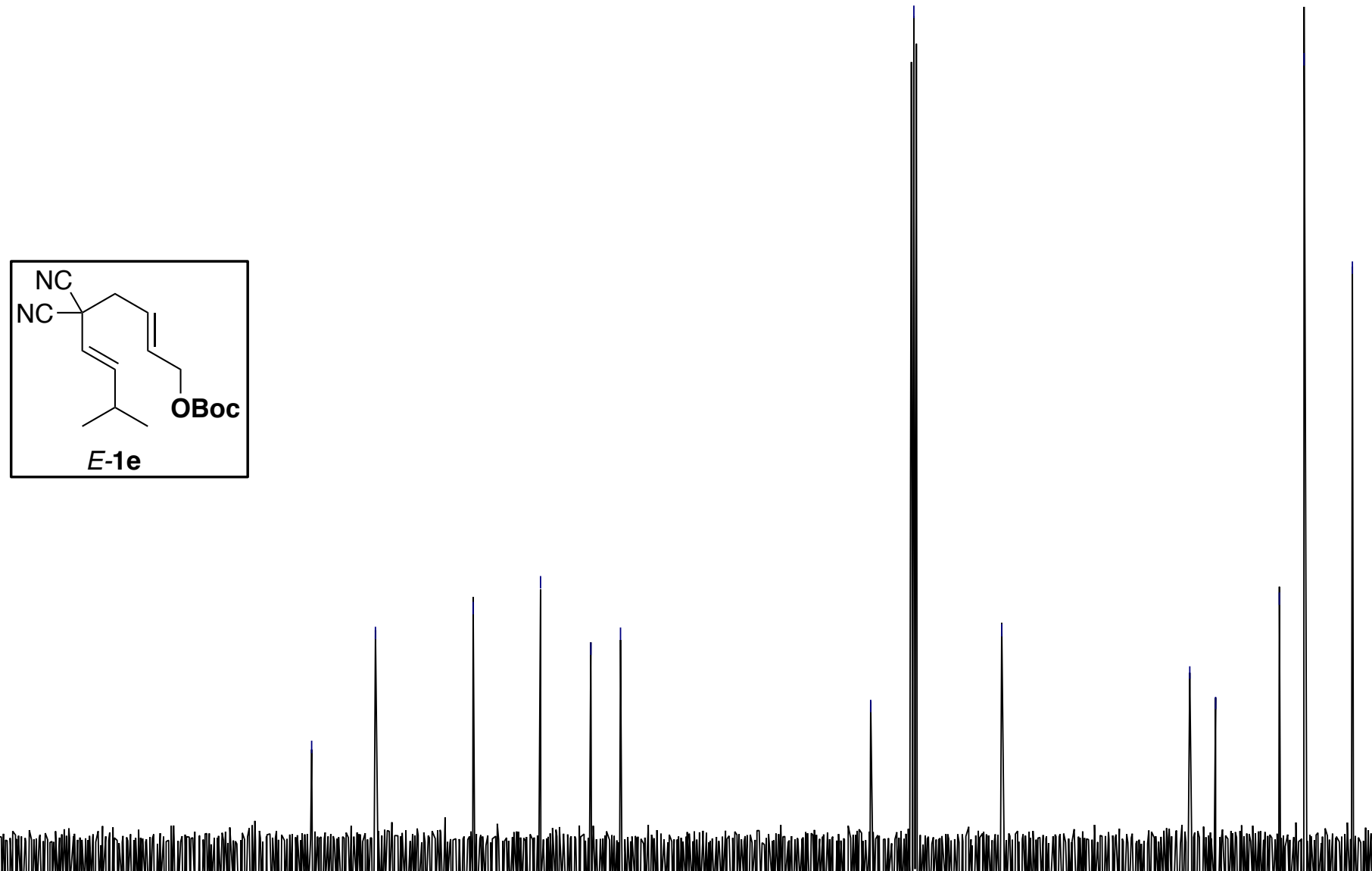

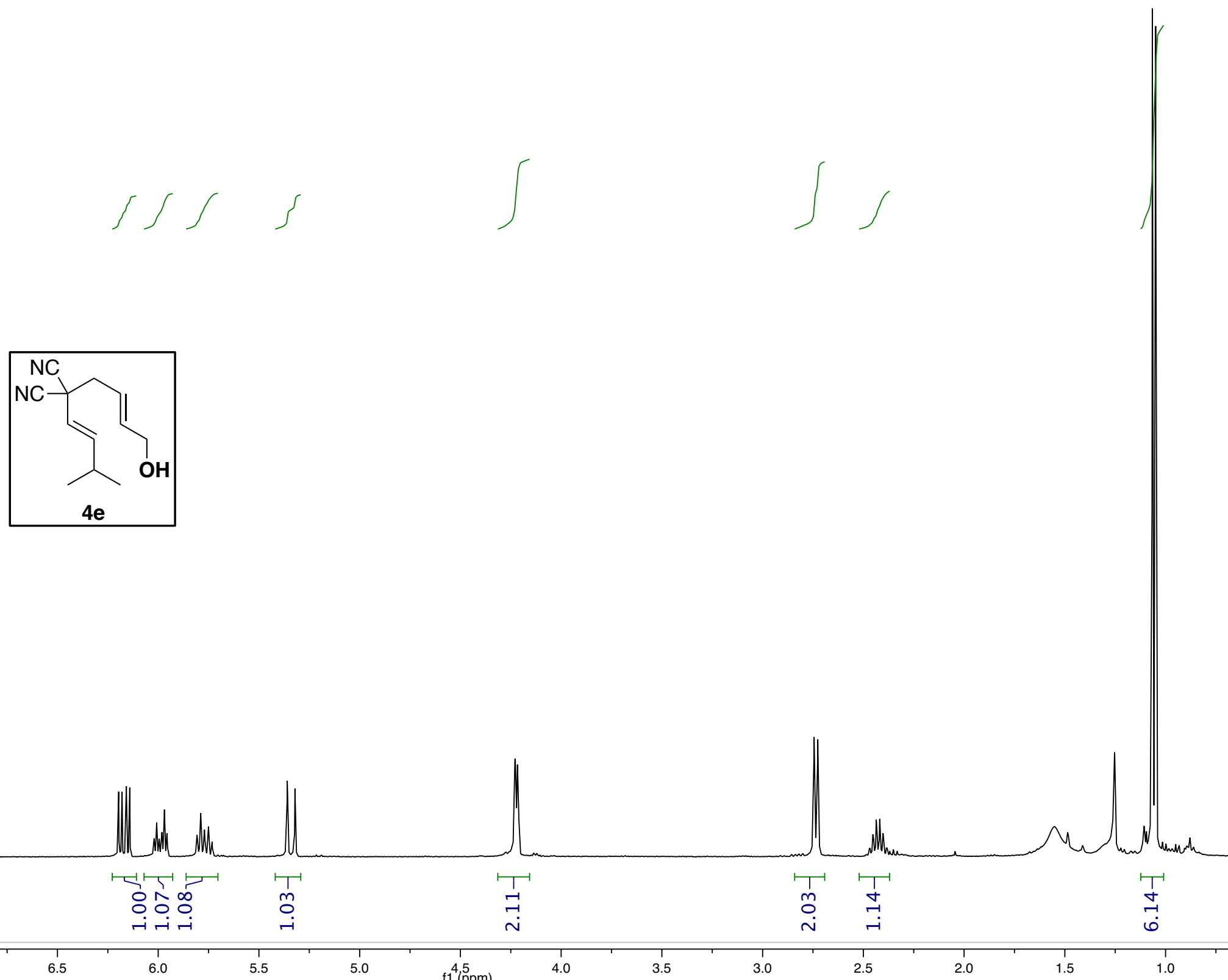


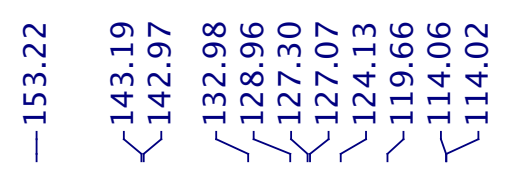

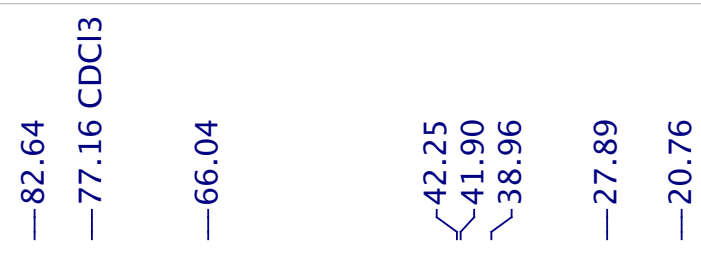

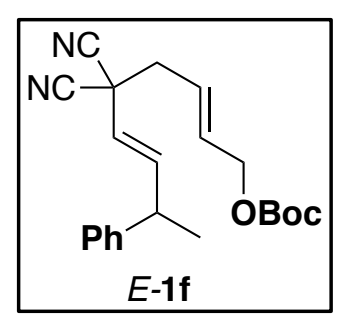

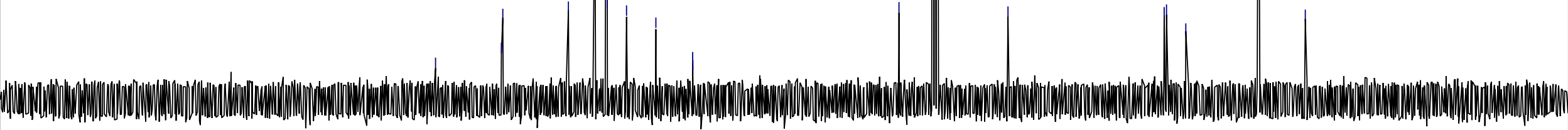

$210 \quad 200$

190

$90 \quad 180$

170

160

150

140

120

100
$\mathrm{f} 1(\mathrm{ppm})$

90

80

70

60

50

40

30

20 


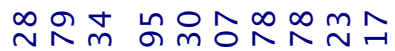

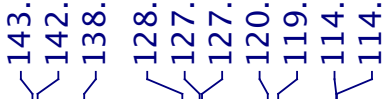

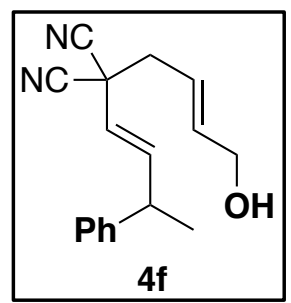

$4 f$

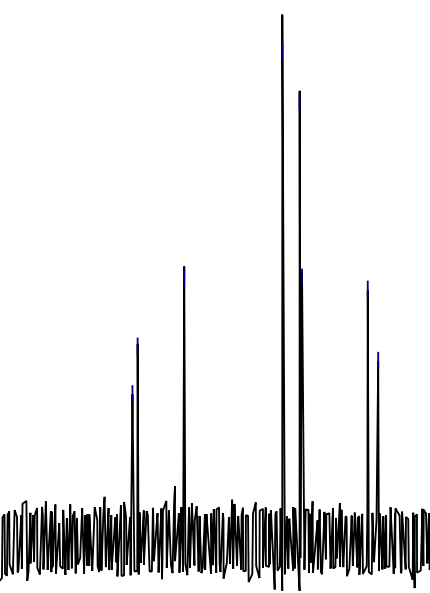


$\frac{m}{U}$
0
0

$i$

|11
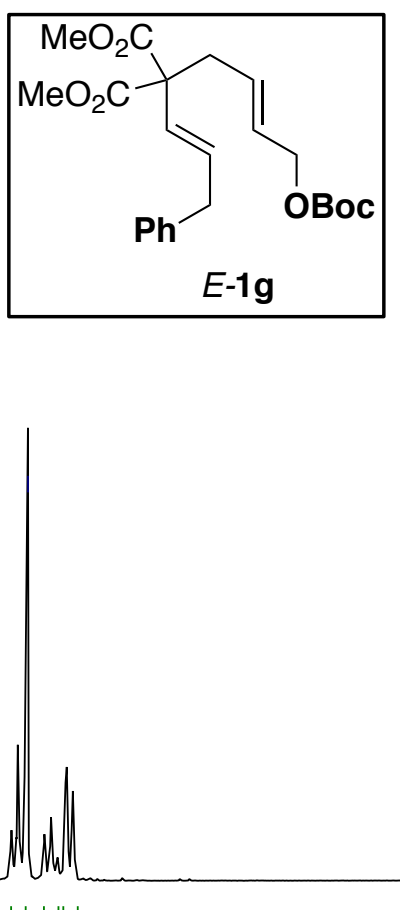

ग्ञ ग्ञात्र

ํㅜㅇㅇ․

i

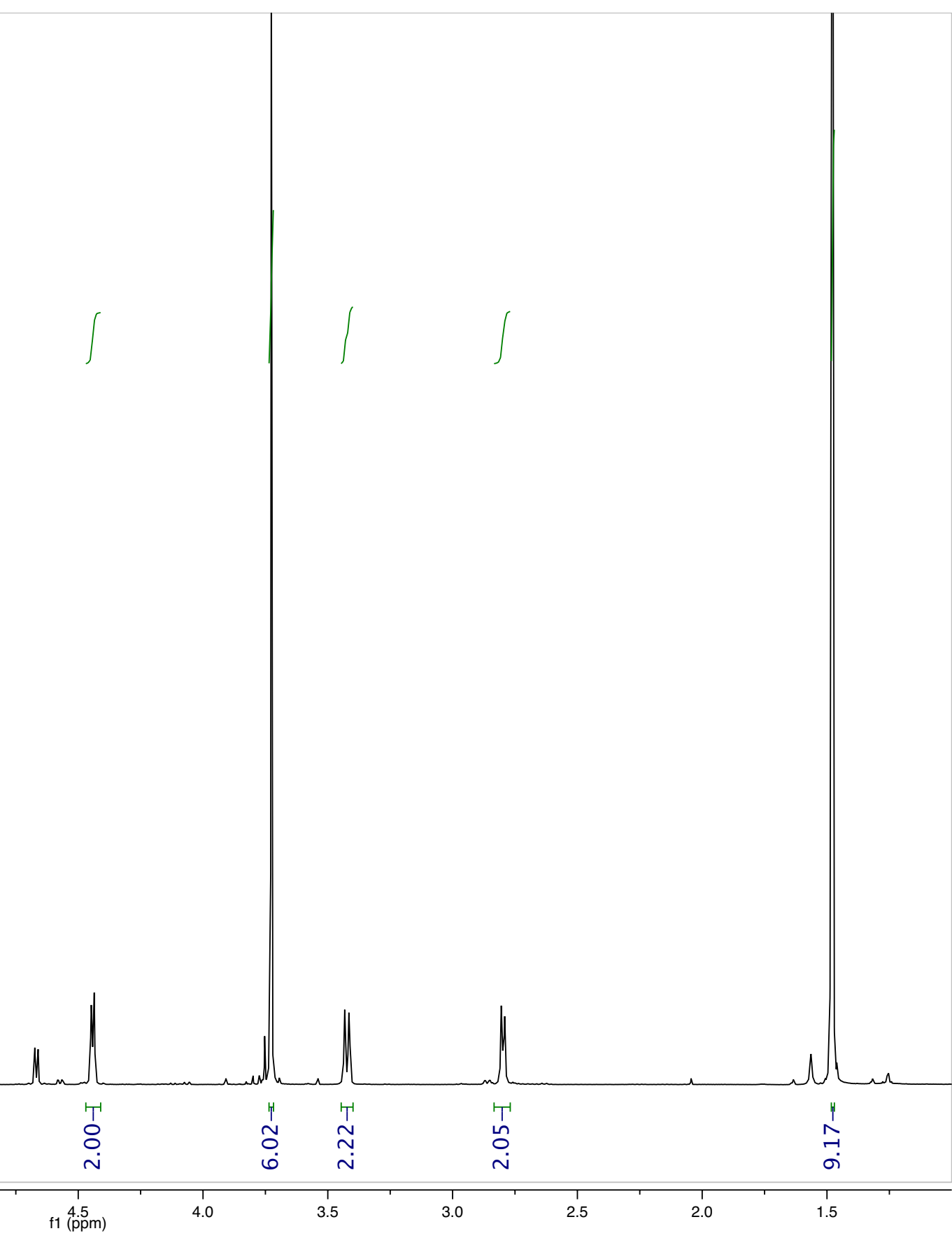




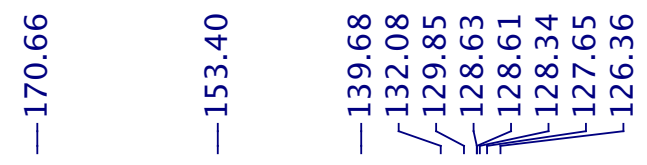

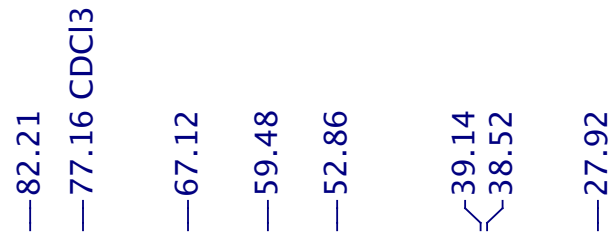
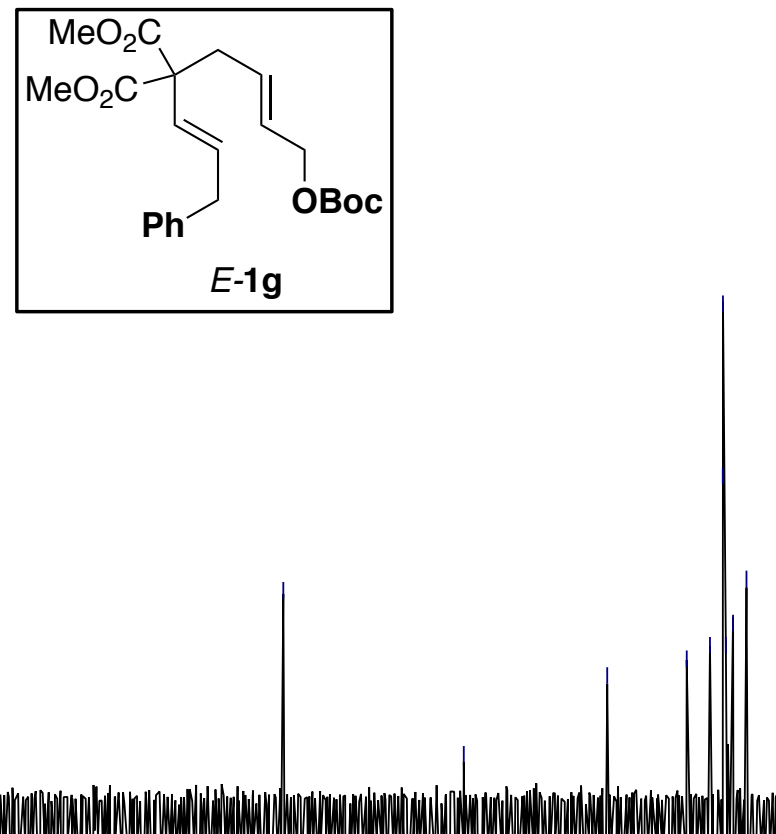


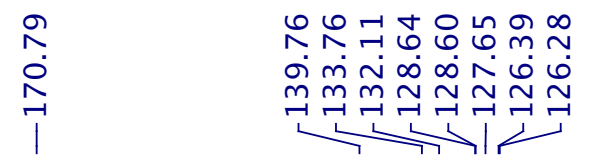

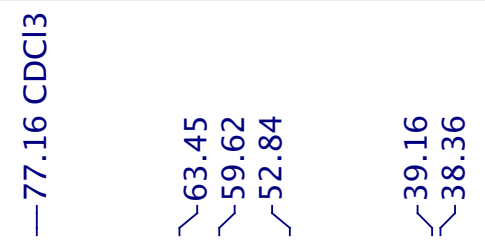
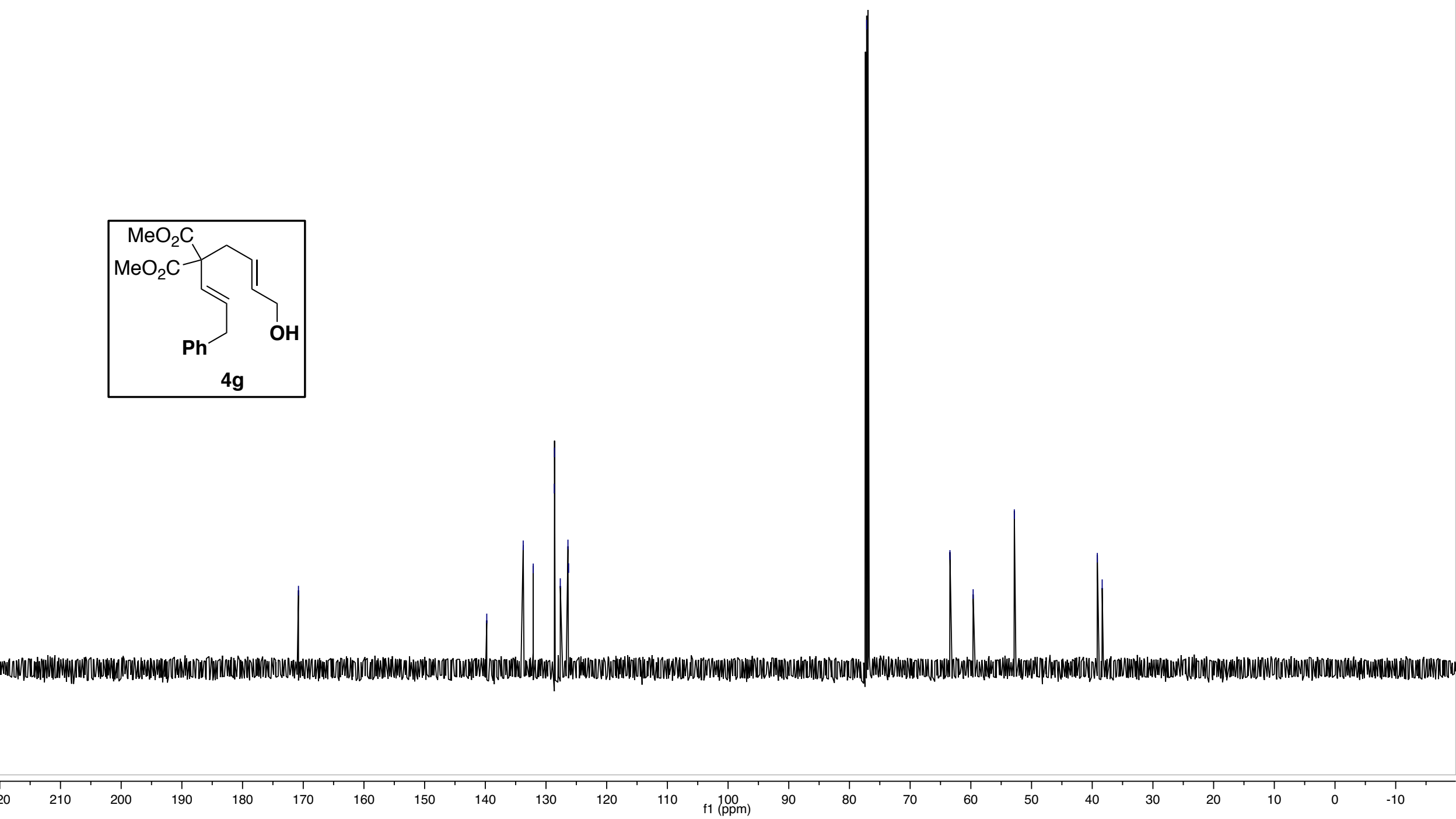
$\frac{m}{U}$
0
0

$i$

$1 / 1$
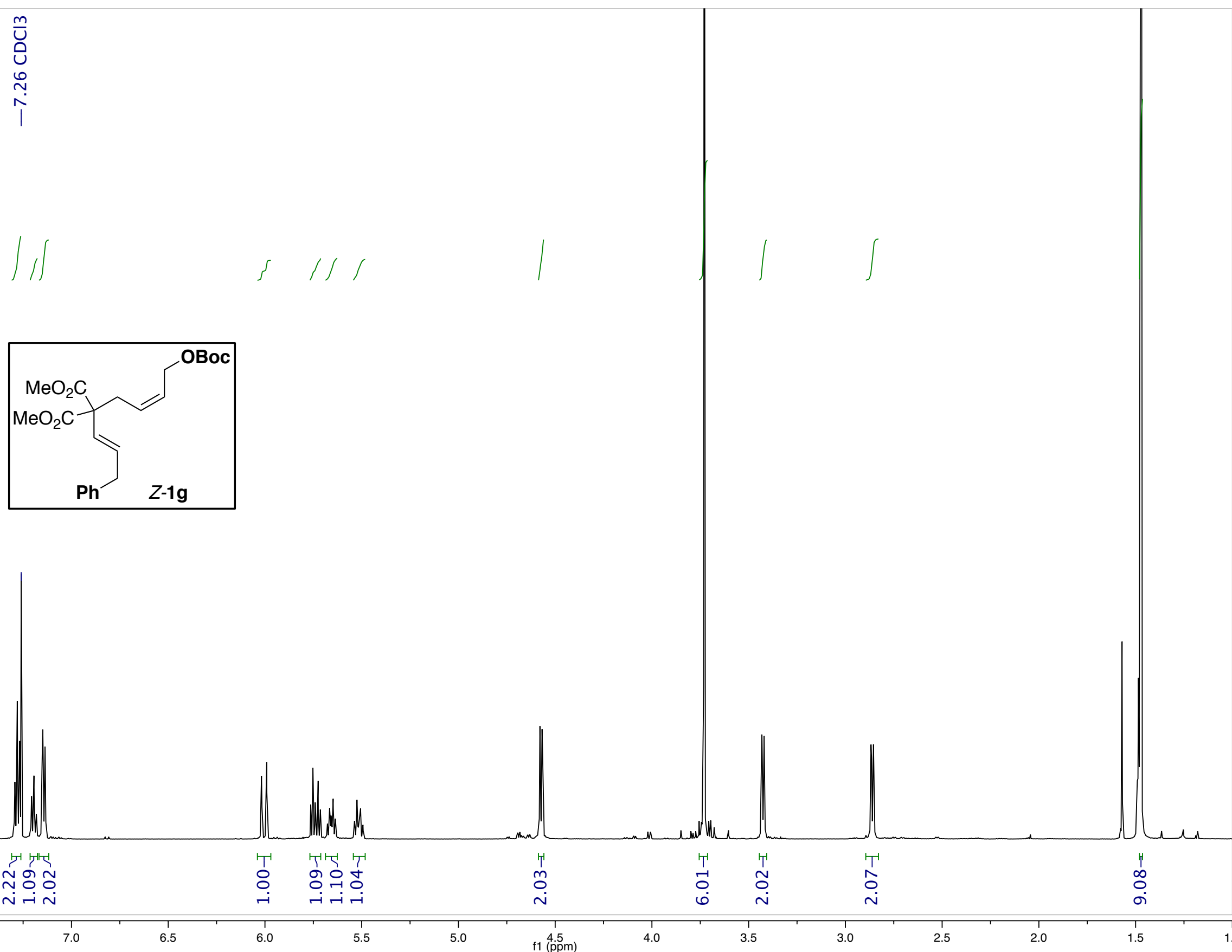


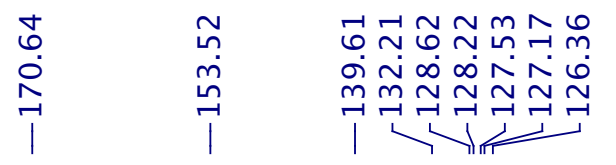

บั

ヘ

ก

ธัํำ

ก $N$

ํำ

i

ไै เ เ

กิm

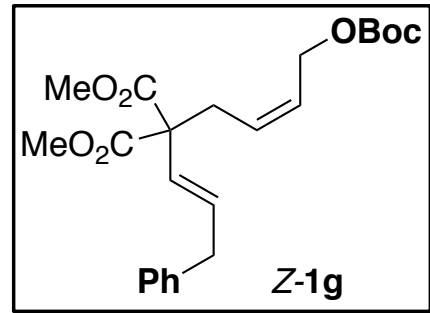

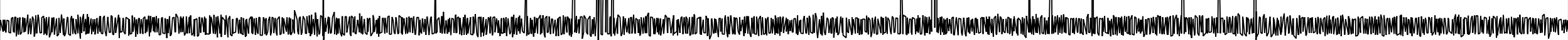
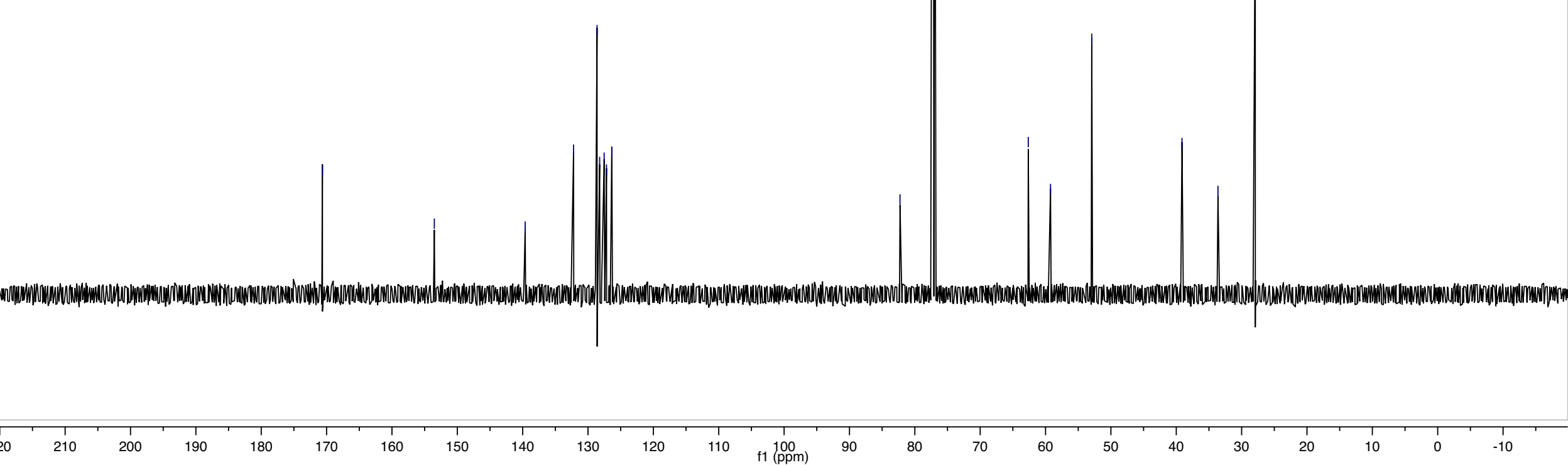


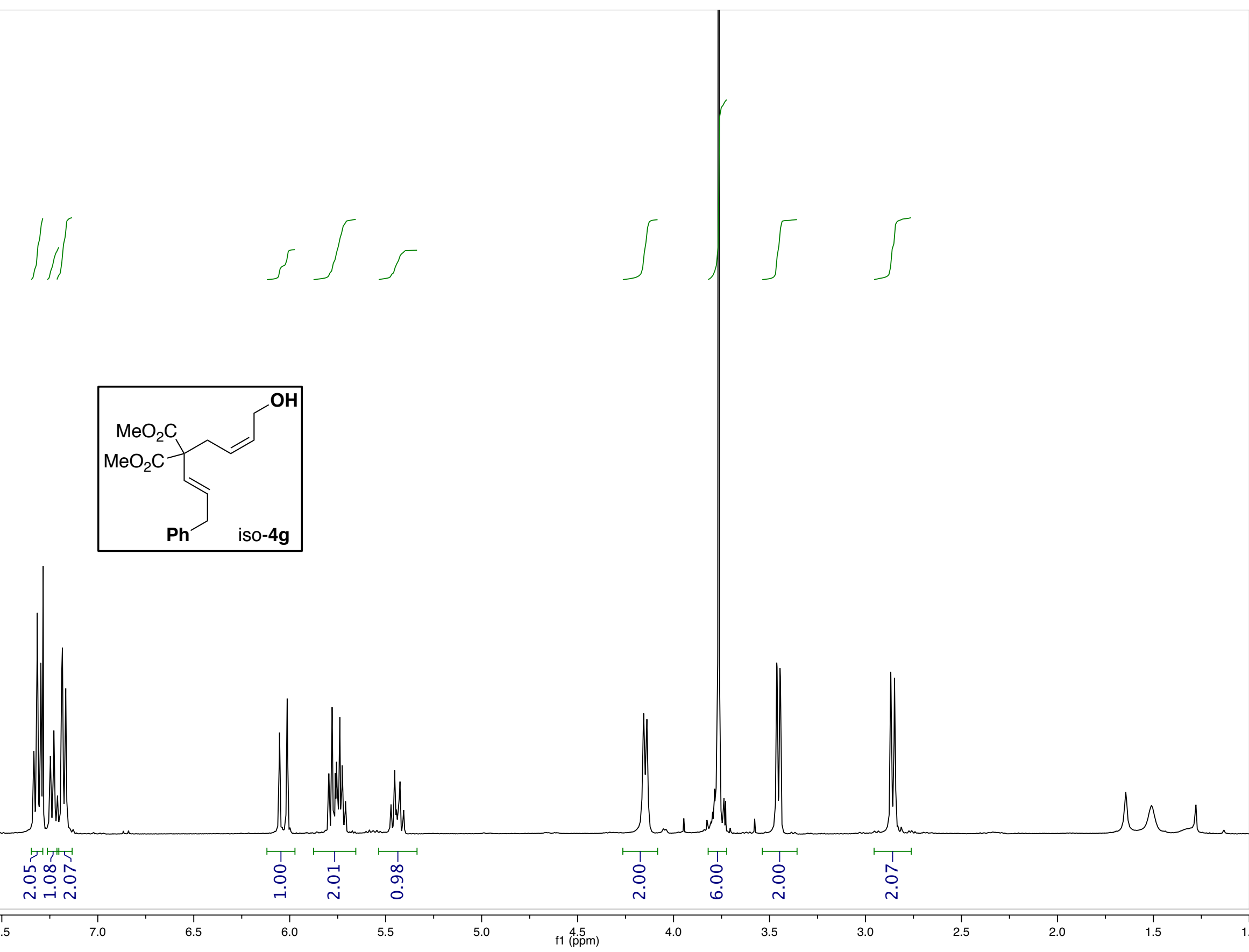



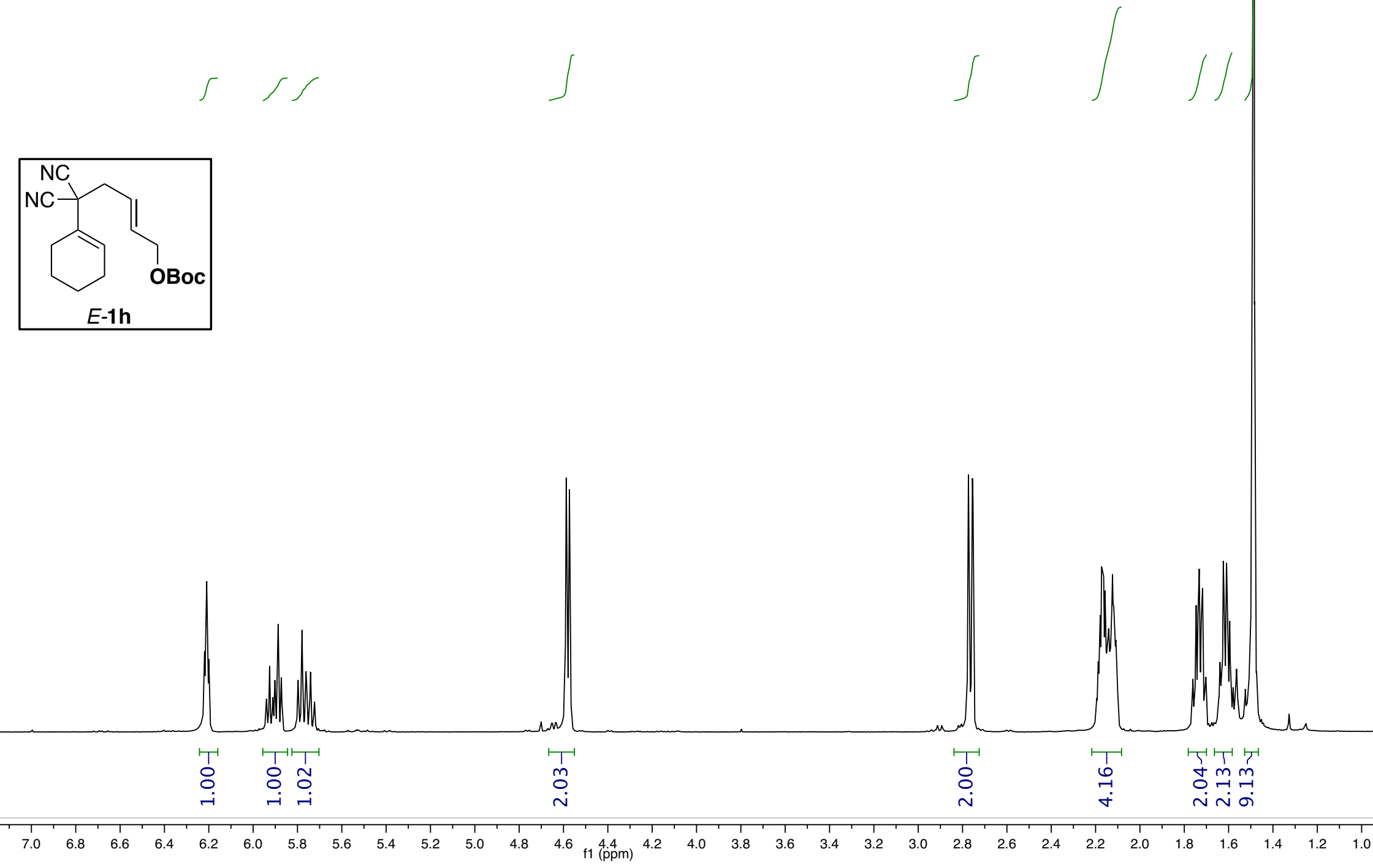


$$
\theta
$$



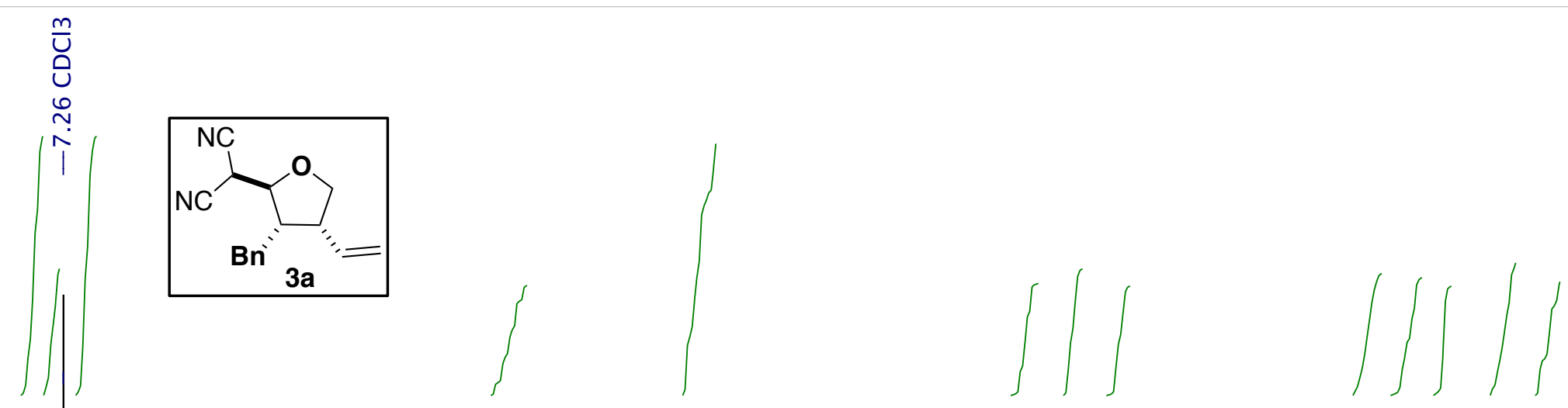

5:1 d.r.

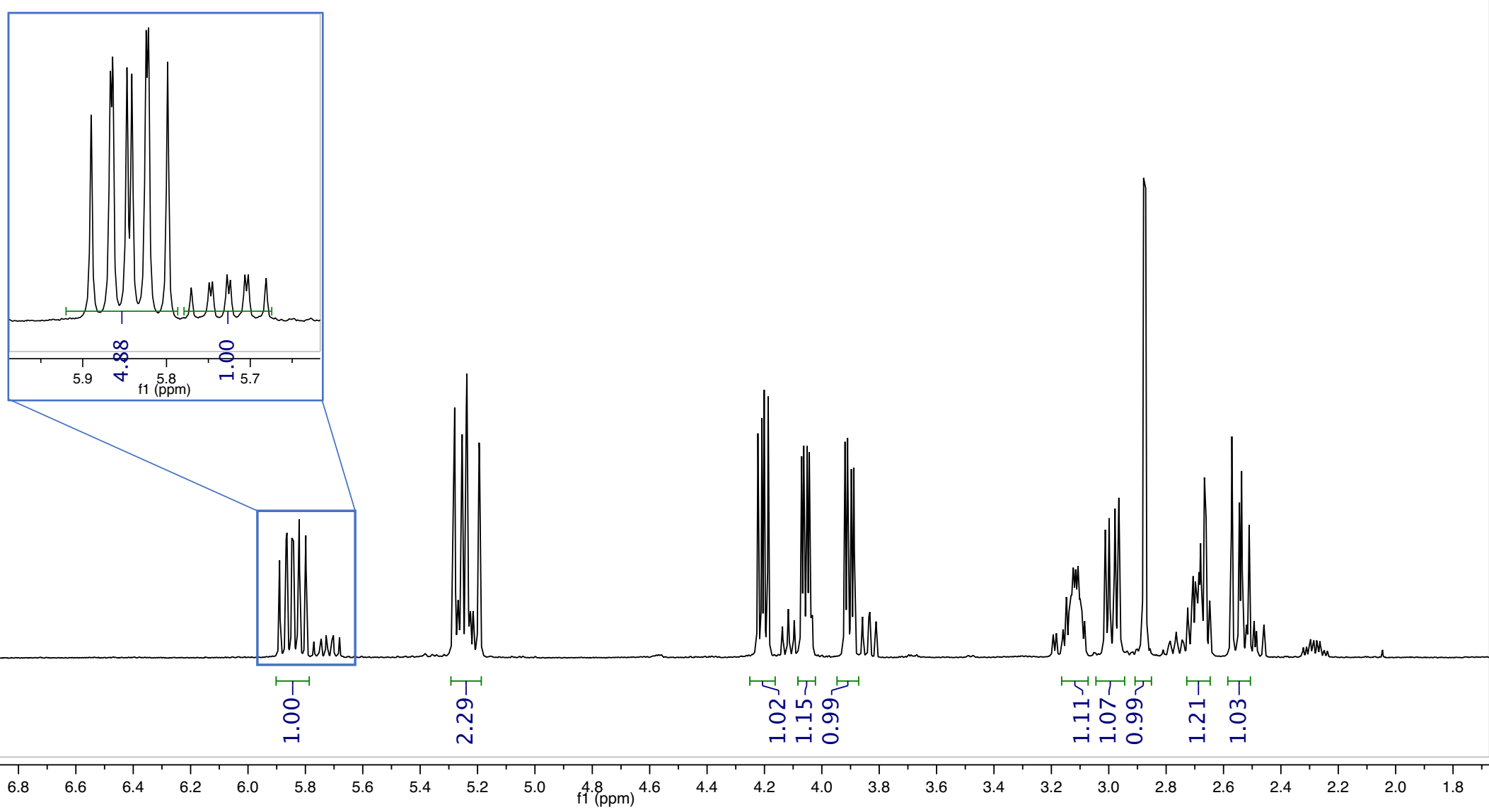




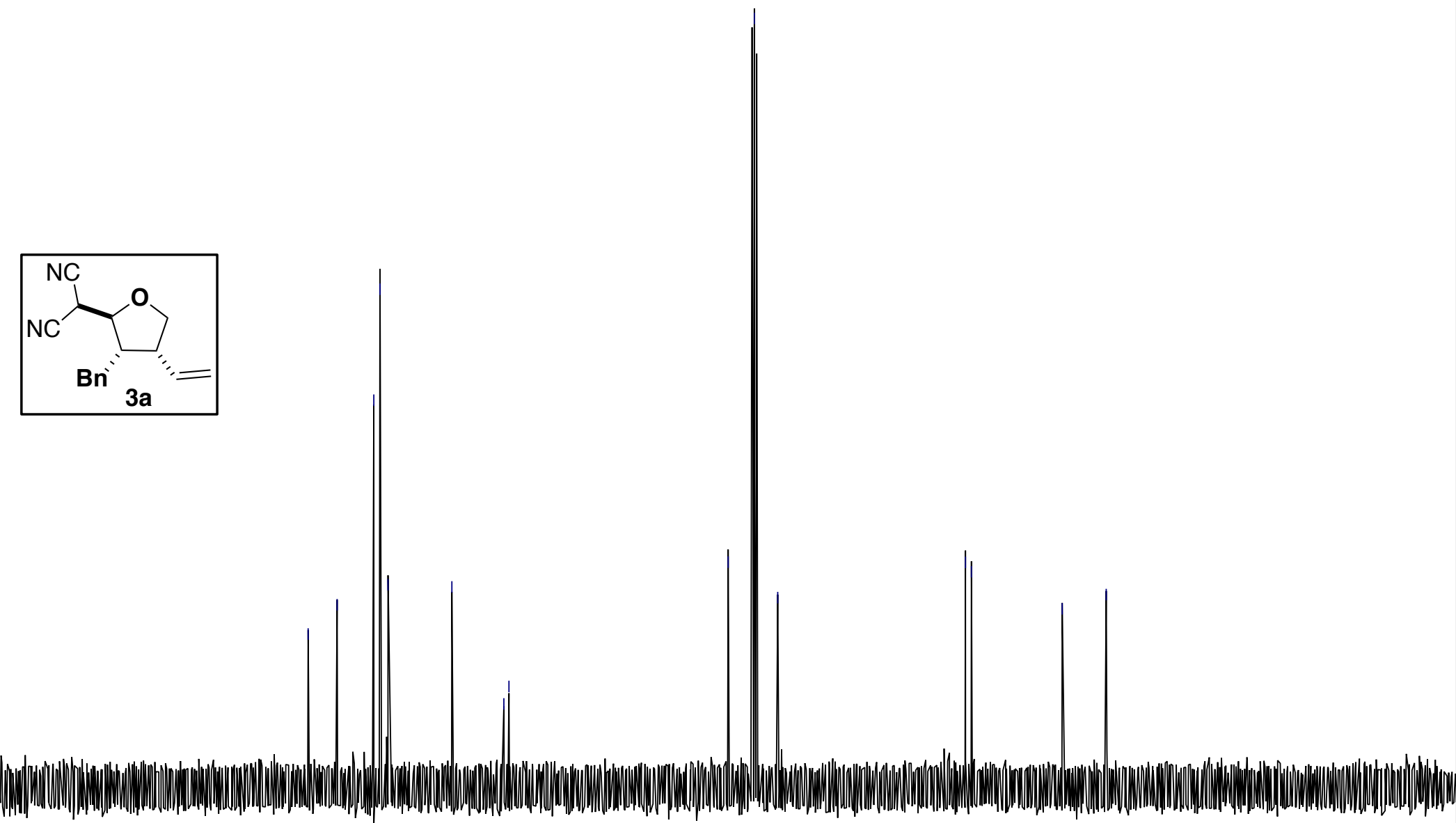




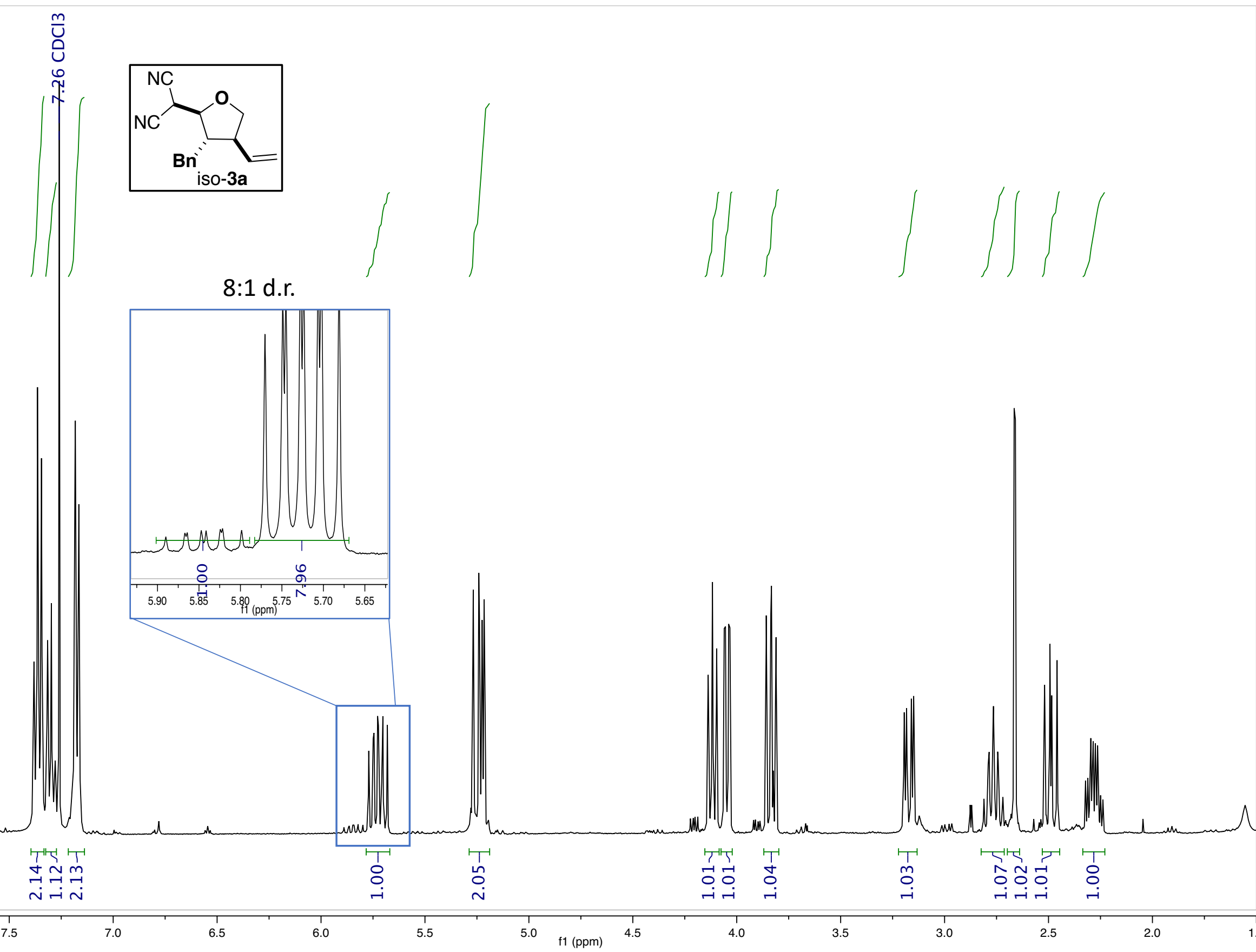




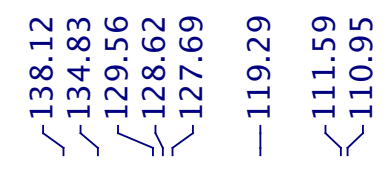

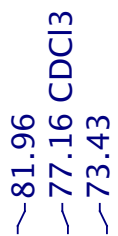

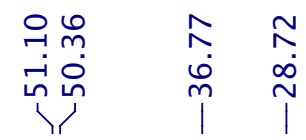

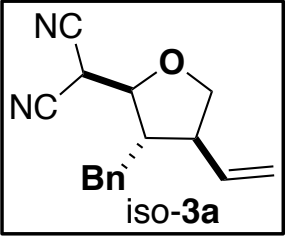



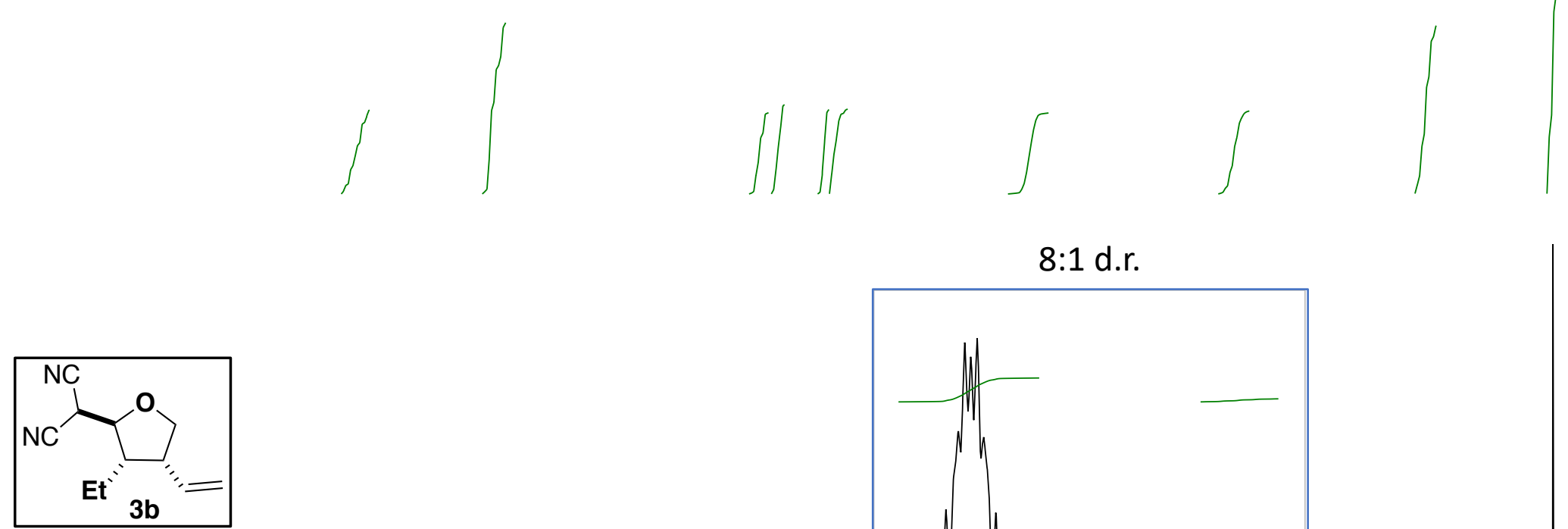

8:1 d.r.
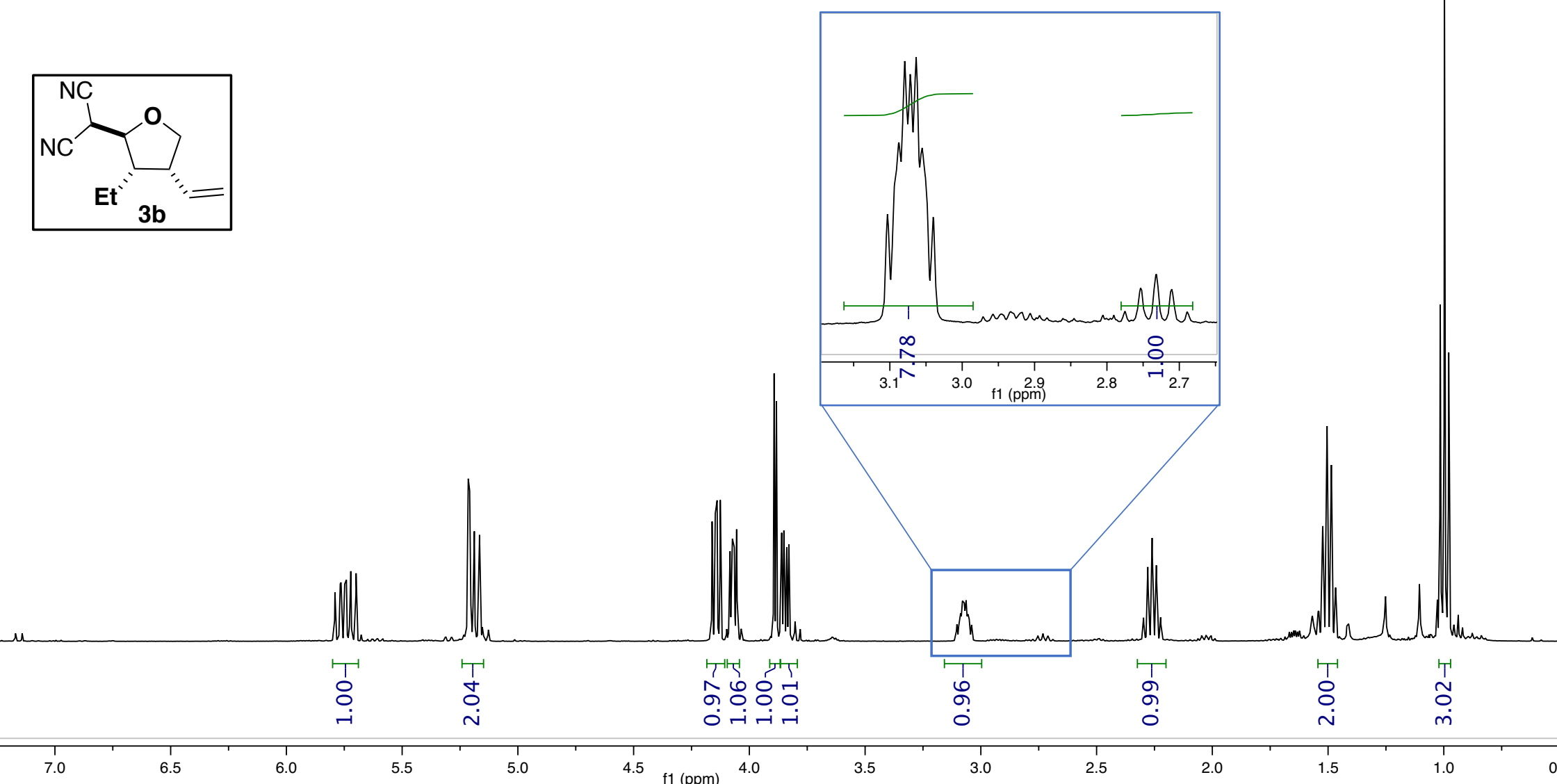


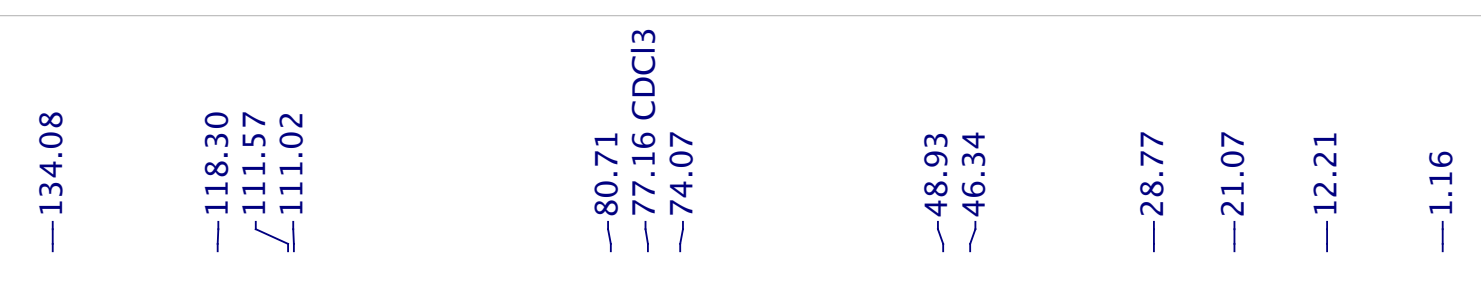

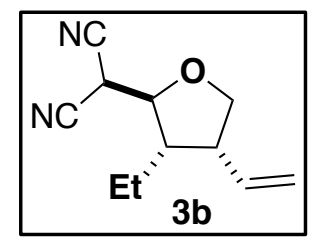


$\frac{m}{\cup}$
0
0
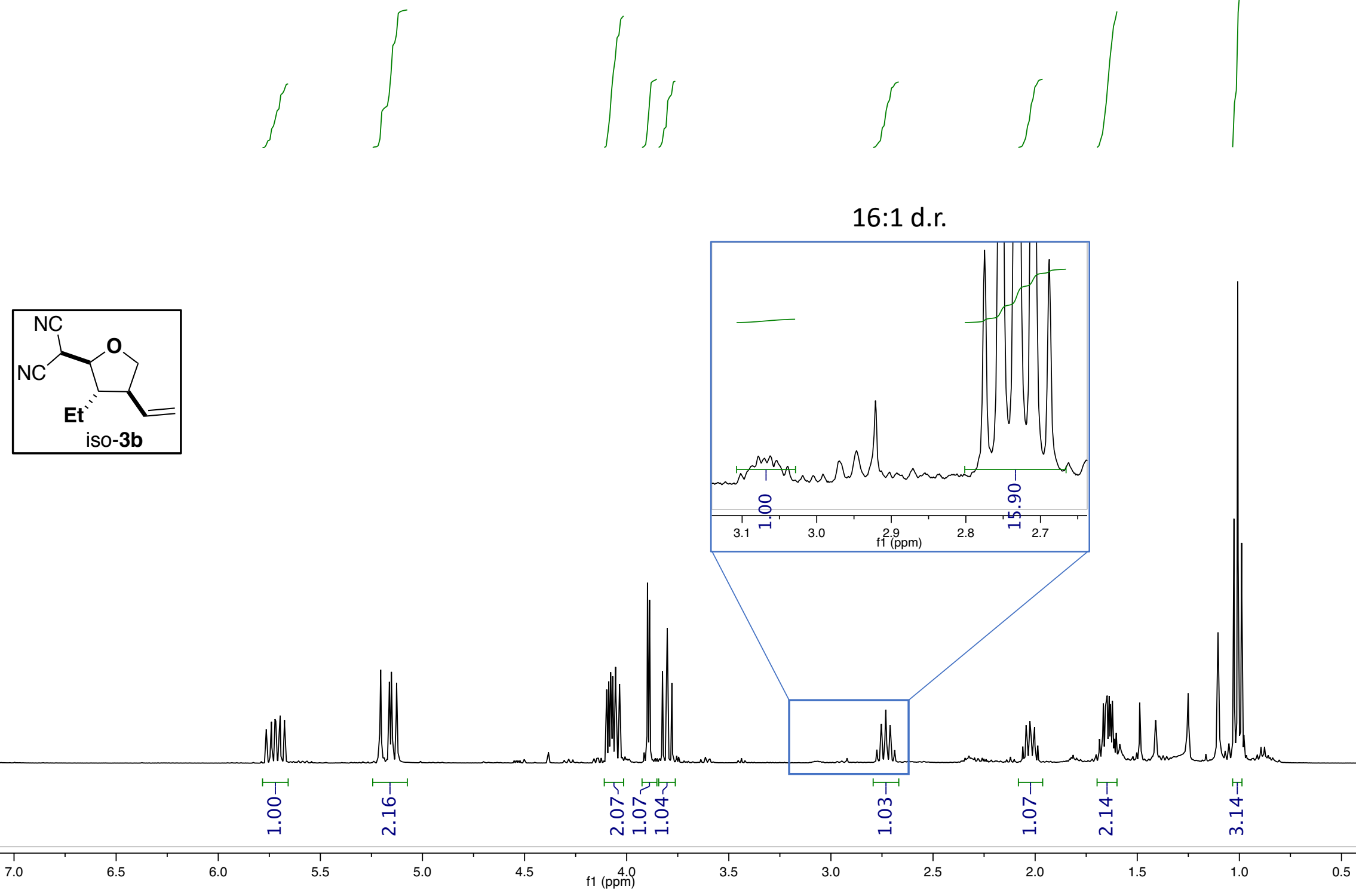


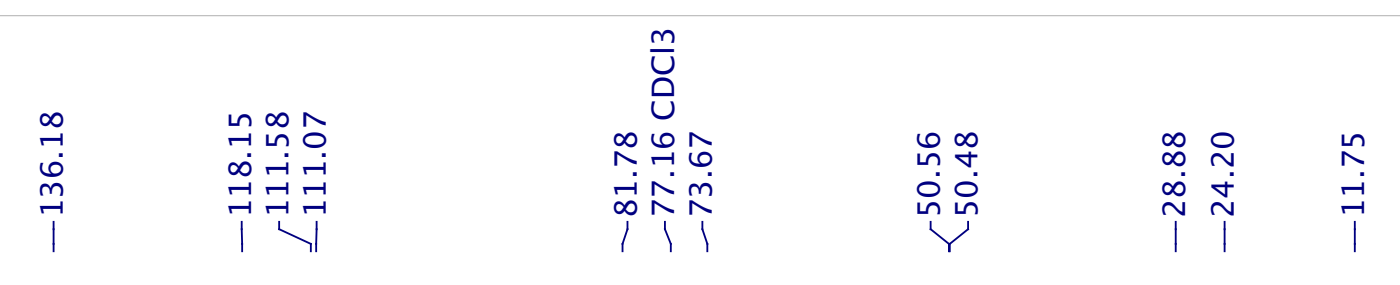

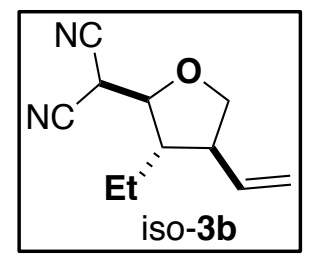

$210 \quad 200$

$\begin{array}{rr}1 & \\ 190 & 180\end{array}$

170

160

150

140

130

120

100
$\mathrm{f} 1(\mathrm{ppm})$

90

80

70

60

50

40

30

20 
$\frac{m}{U}$
0
0
$\stackrel{n}{i}$
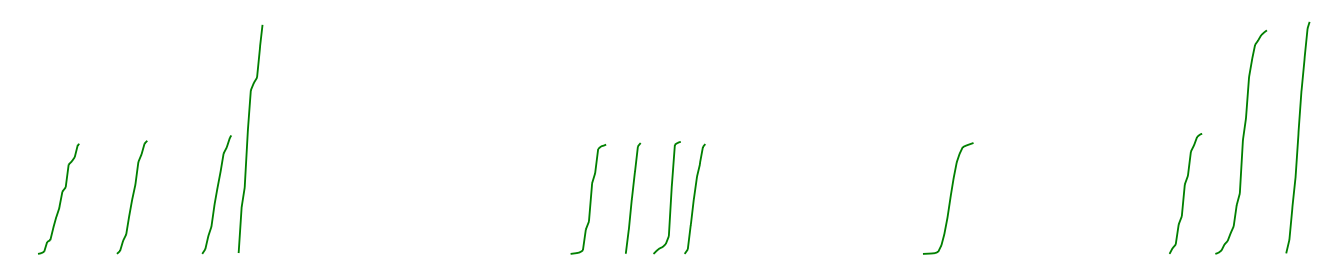

7:1 d.r.
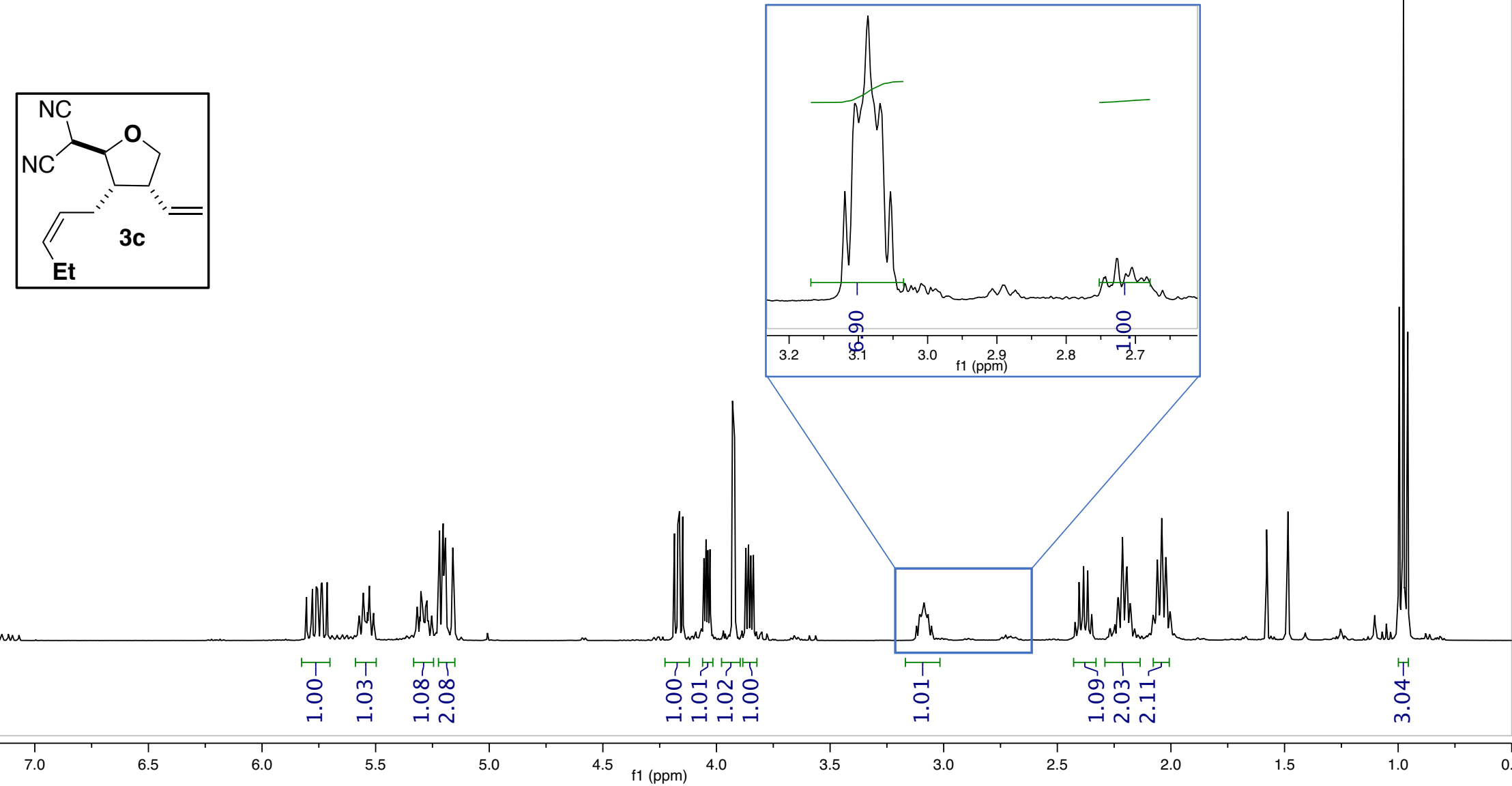


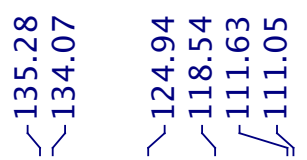

๙ே ホ

$\circ \hat{N}$

$1 \mid$

$\begin{array}{ll}+ & 0 \\ \infty & 0 \\ 0 & 0 \\ \dot{y} & +\end{array}$

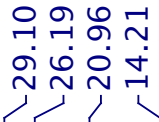
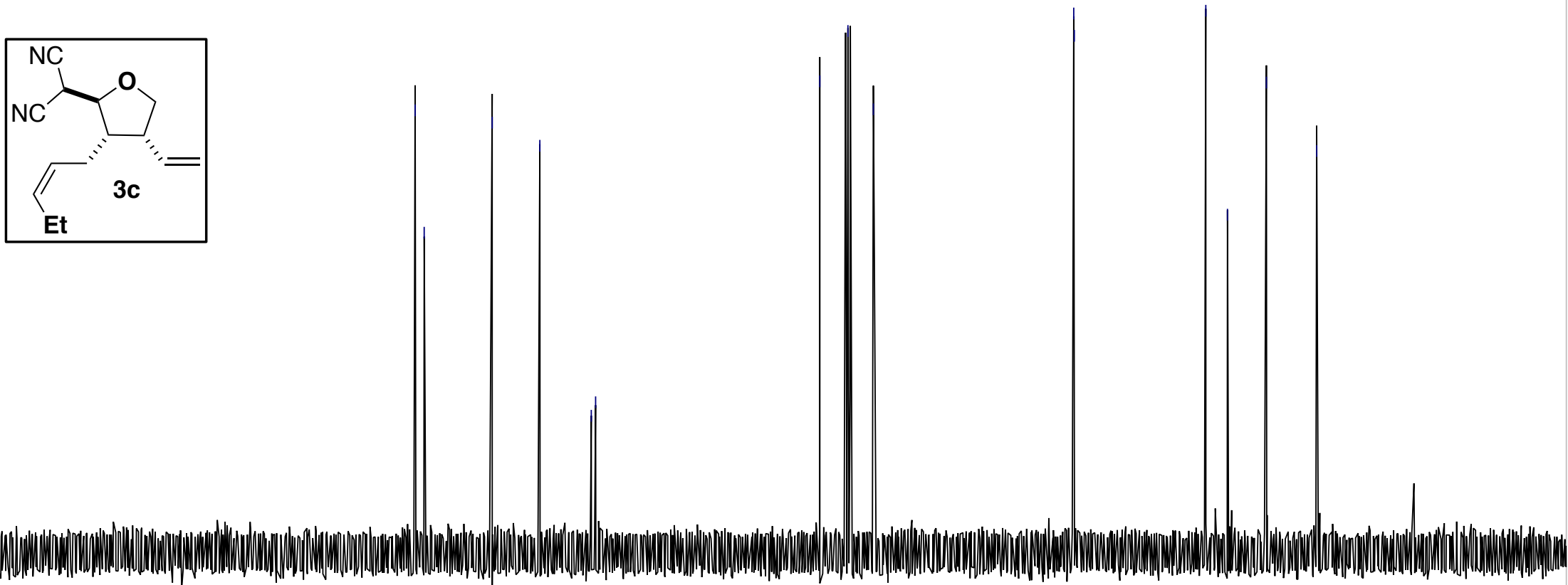

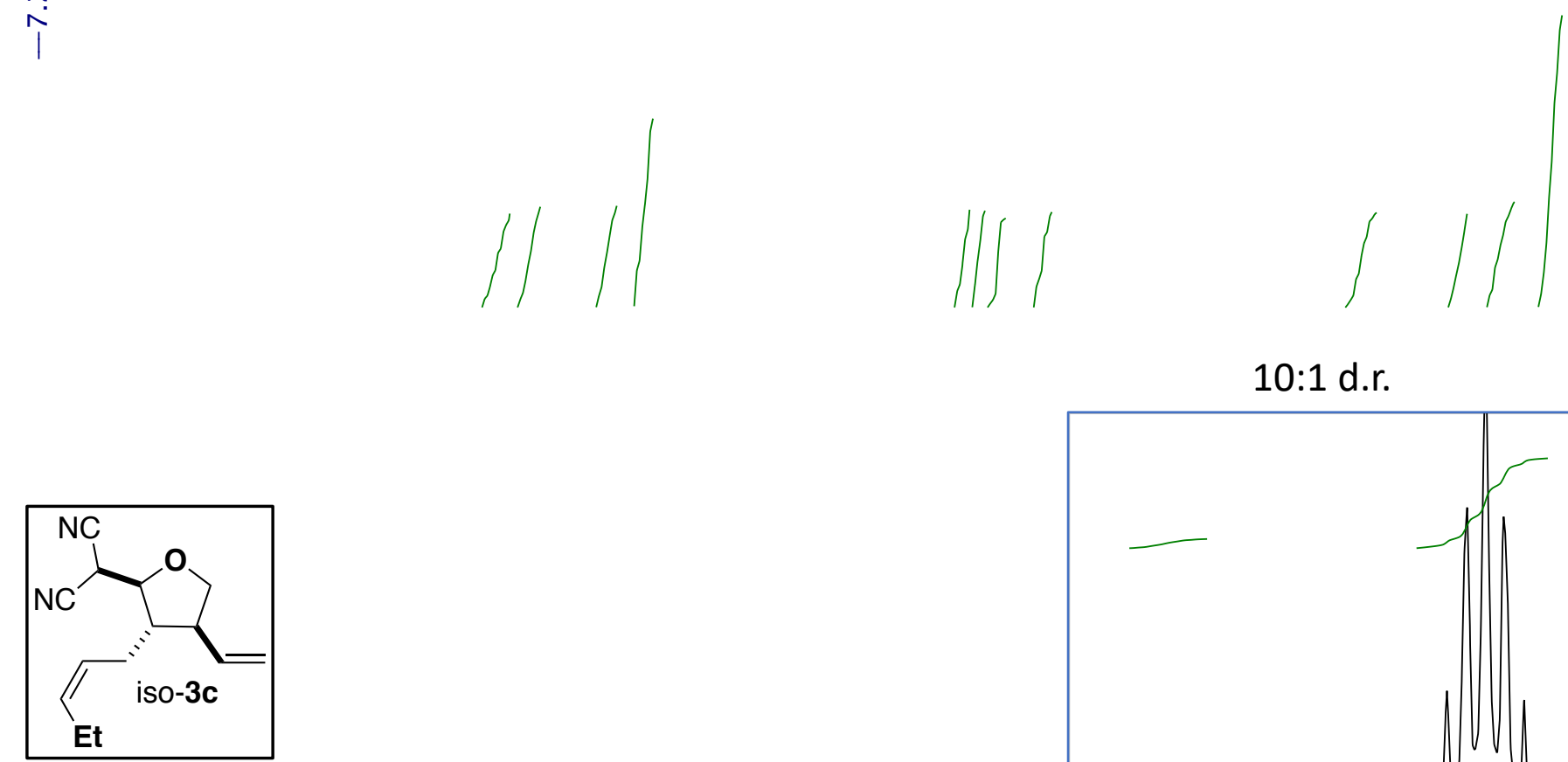

10:1 d.r.

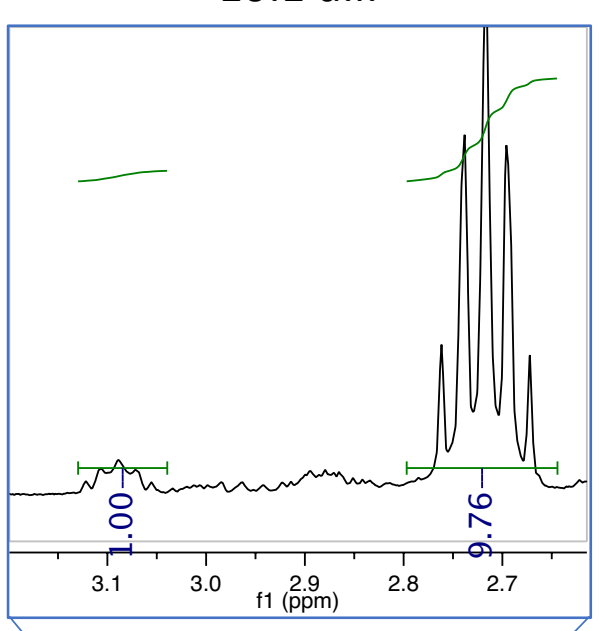

Ith

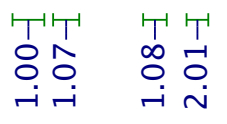

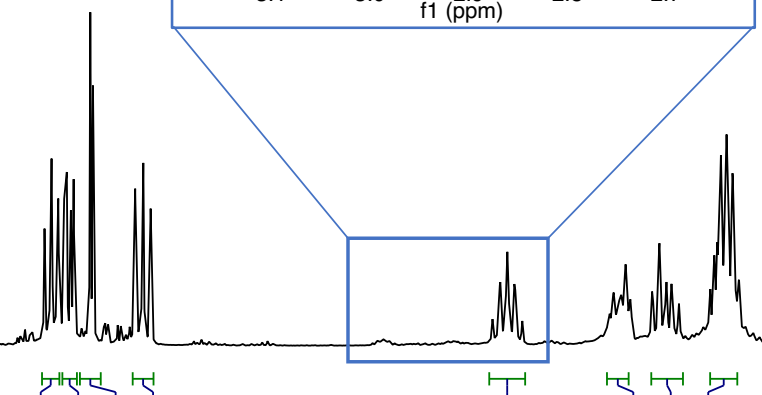

पूल मं

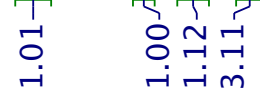




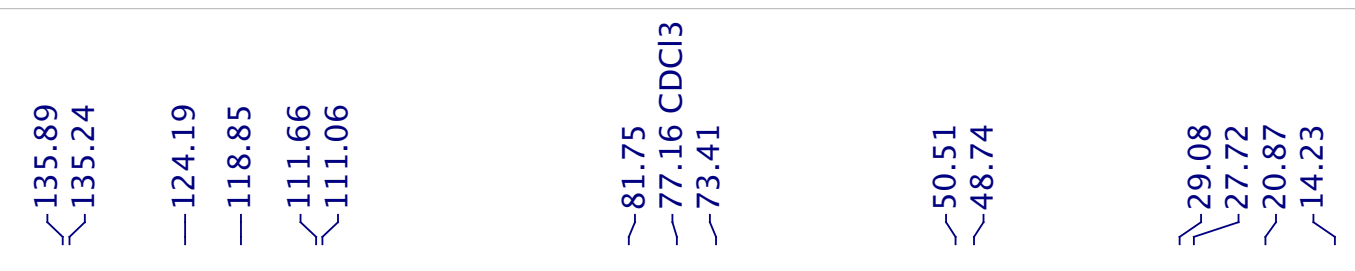

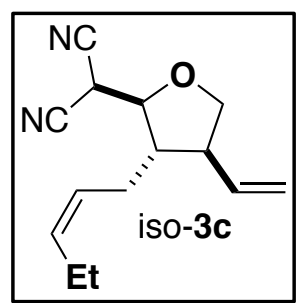

$210 \quad 200$

190

180

170

160

150

140

130

120

100
$\mathrm{f} 1(\mathrm{ppm})$

90

80

70

60

50

40

30

20

10 


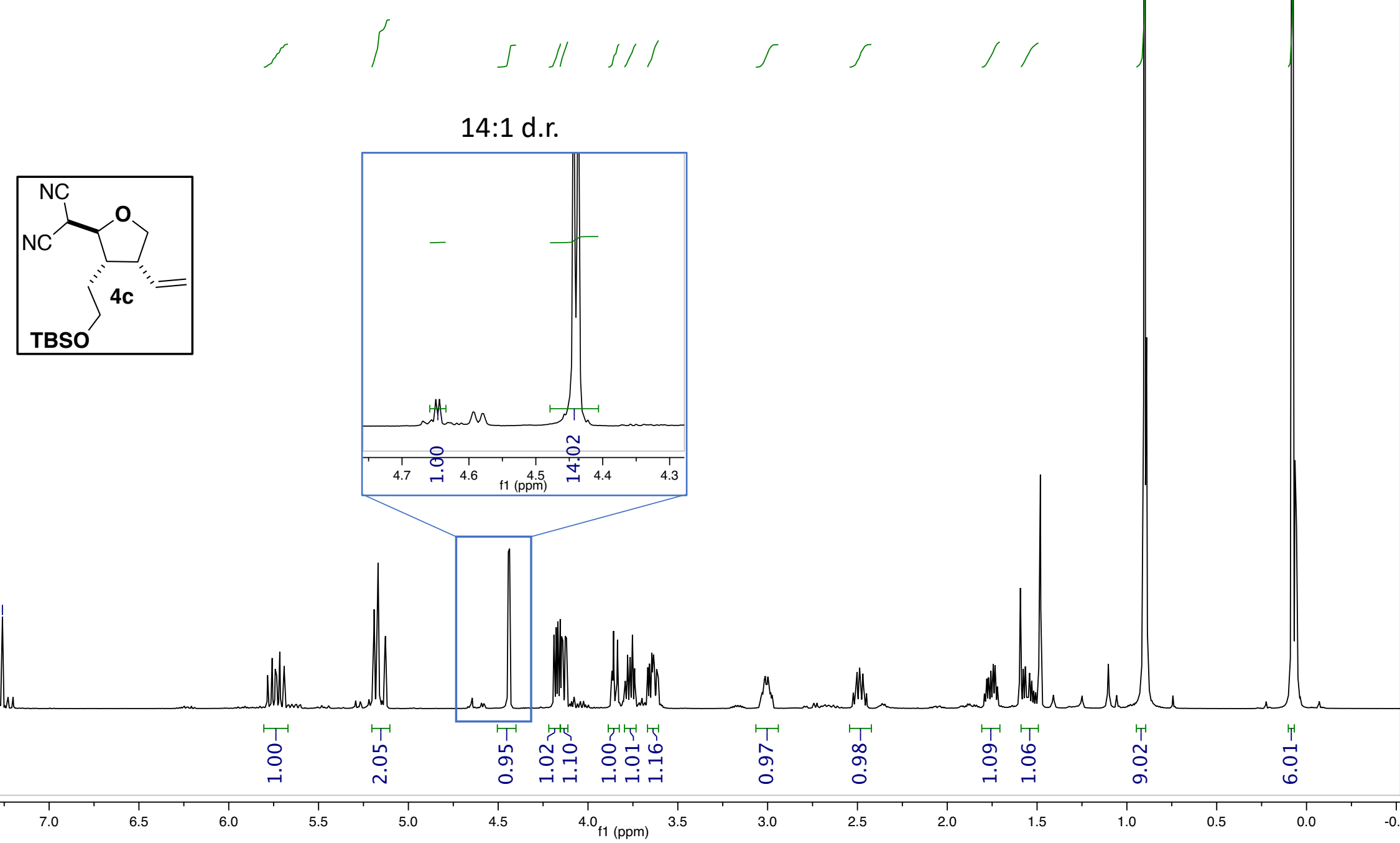




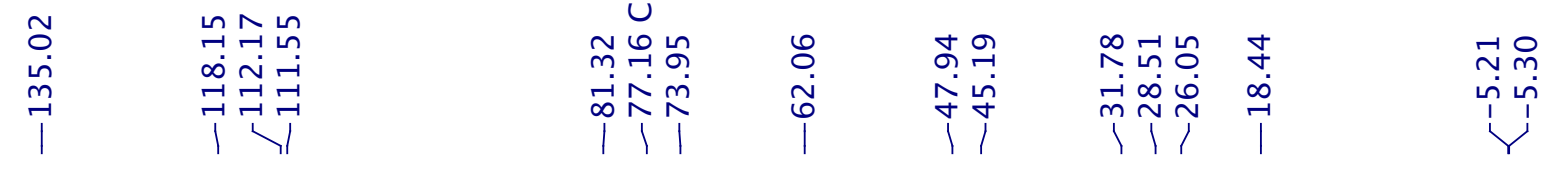

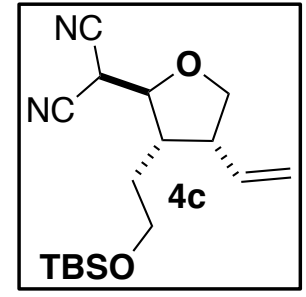

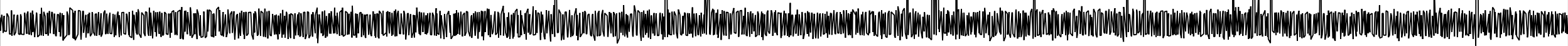

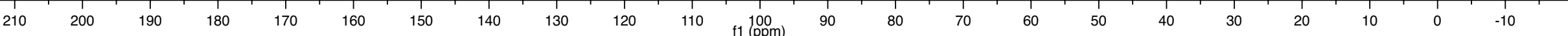




\section{* Trace of Knoevenagel starting material}

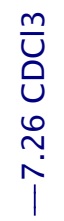
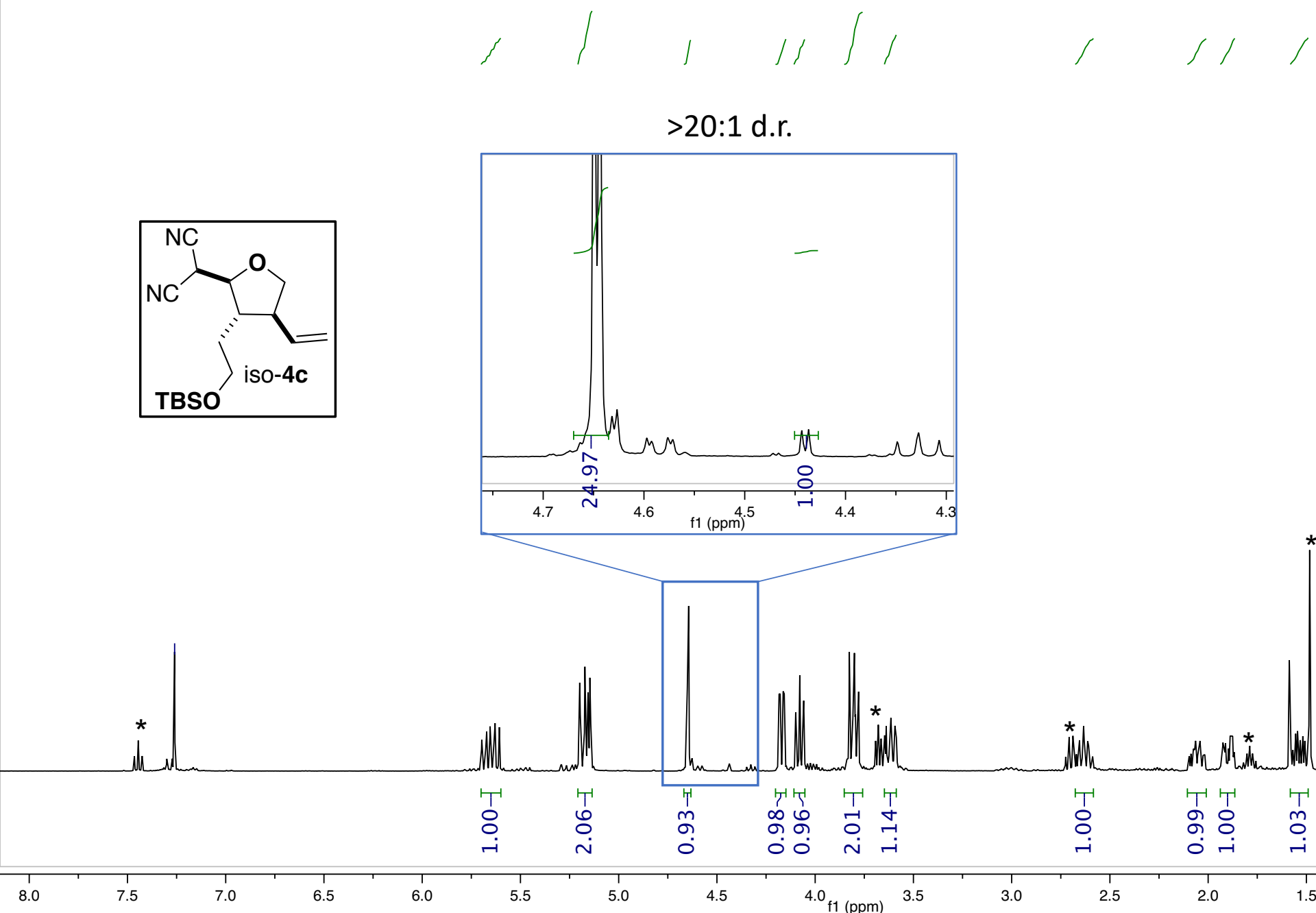

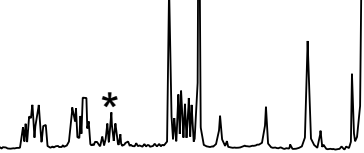

20

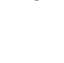




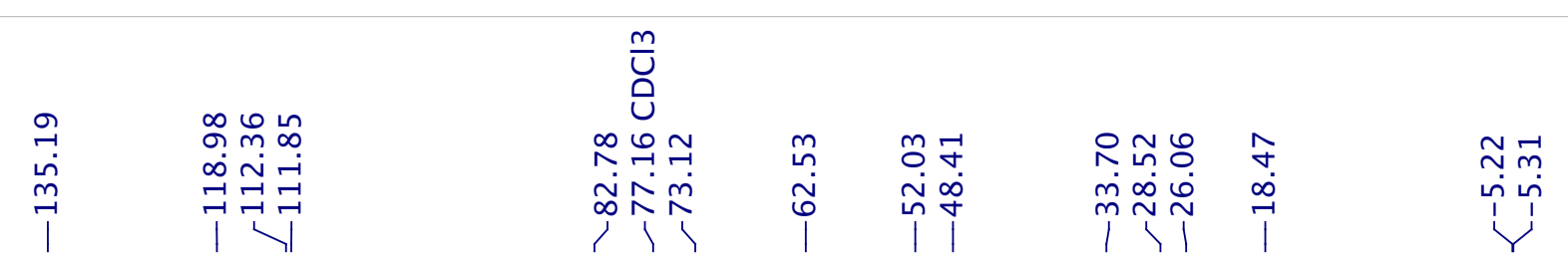

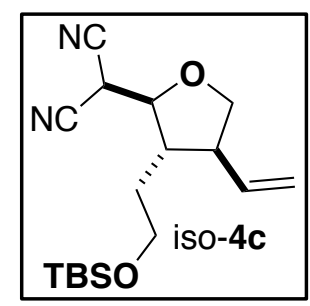



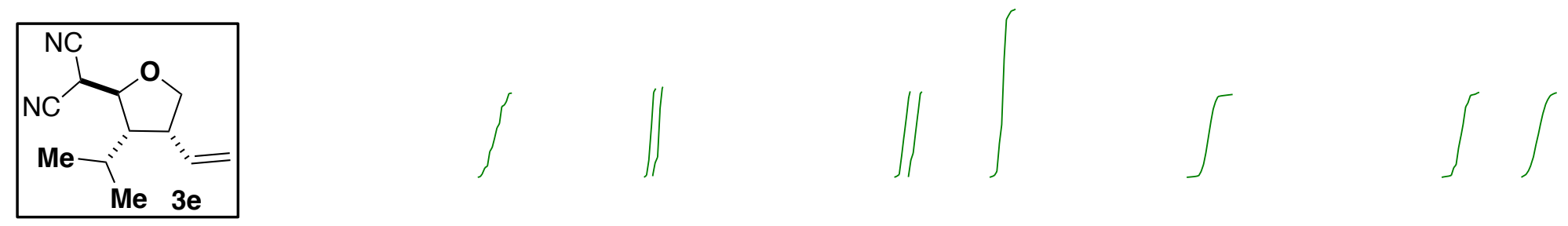

9:1 d.r.

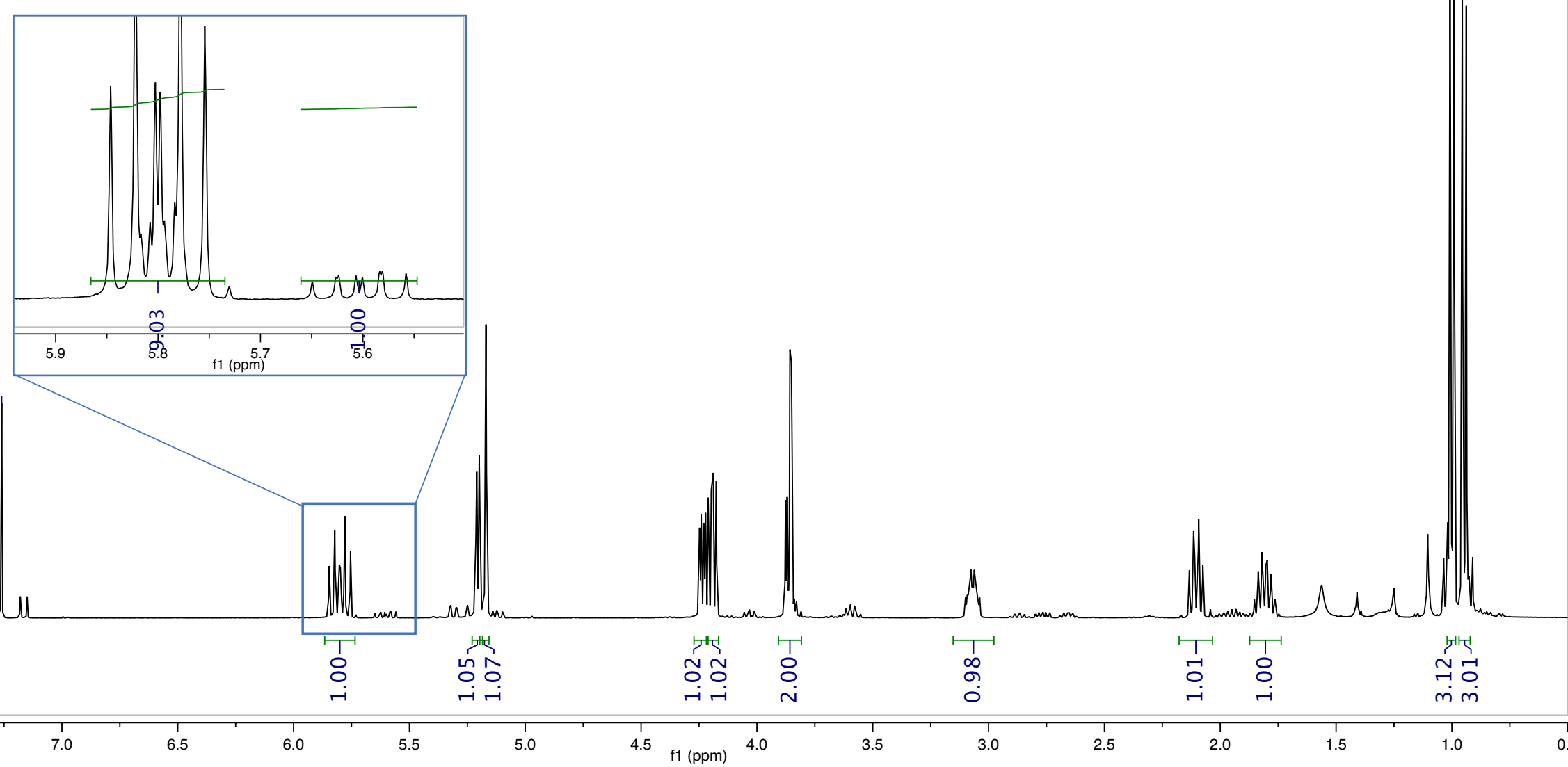




\begin{tabular}{|c|c|c|c|c|}
\hline & & 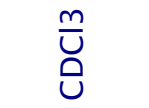 & & \\
\hline $\begin{array}{c}\stackrel{+}{+} \\
\dot{\vec{p}} \\
\vec{T} \\
\overrightarrow{1}\end{array}$ & 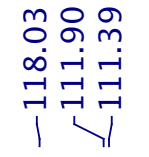 & 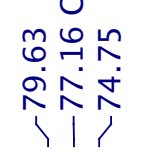 & $\begin{array}{ll}\hat{N} & \overrightarrow{+} \\
\hat{n} & \stackrel{+}{+} \\
\hat{\jmath} & 广\end{array}$ & 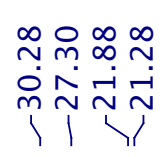 \\
\hline
\end{tabular}

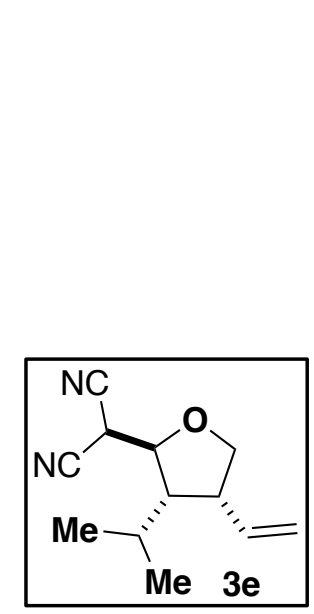

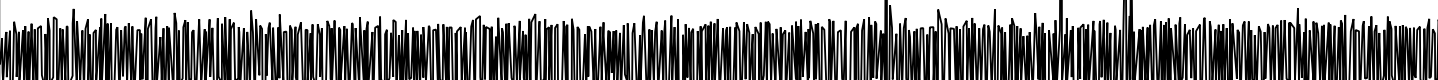
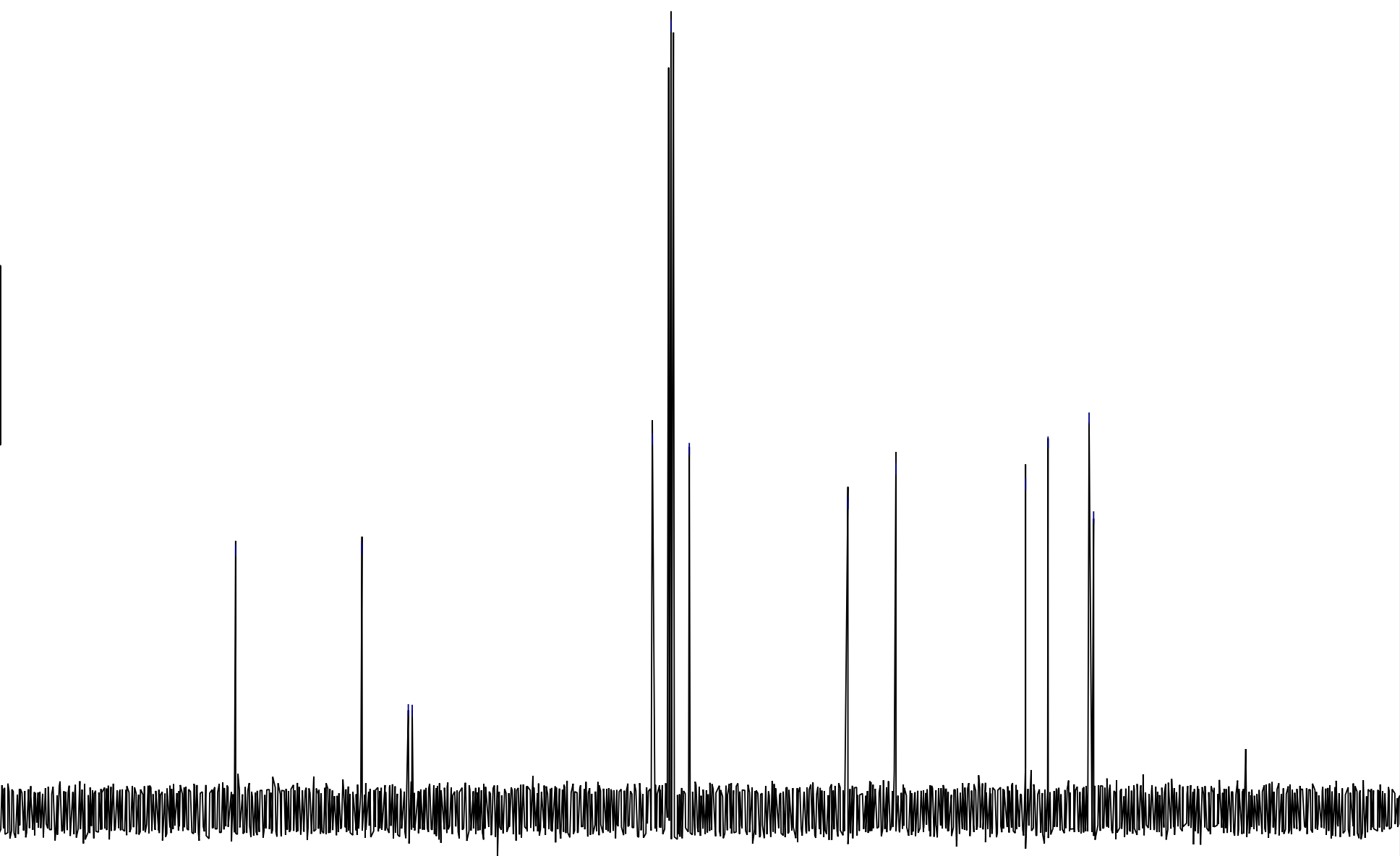

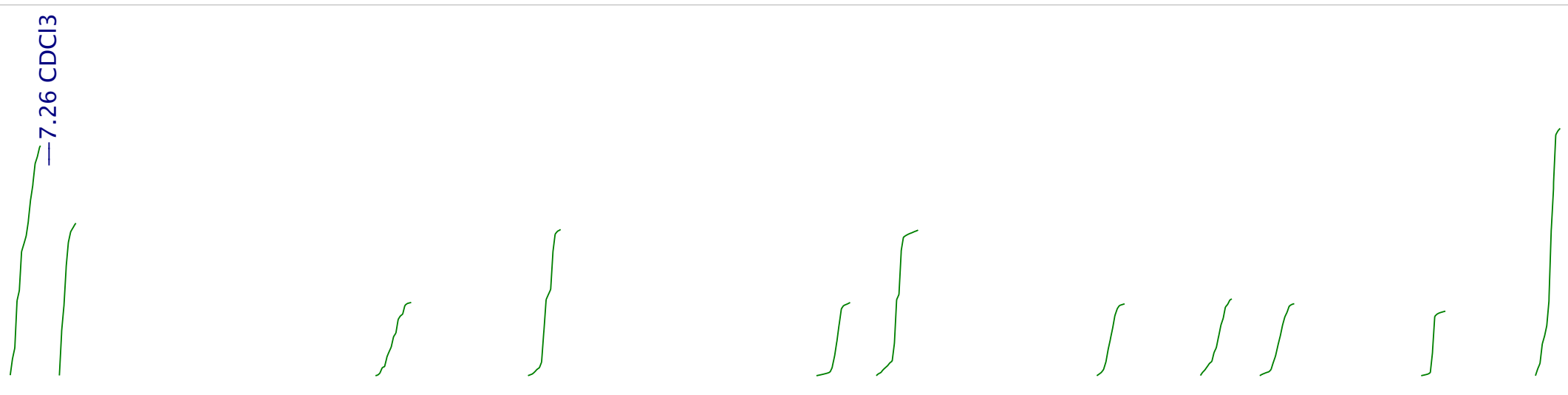

\section{Diastereomer 1}

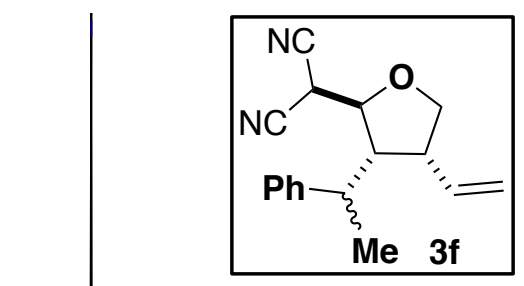

WNWWh

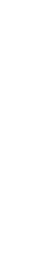




\section{Diastereomer 1}

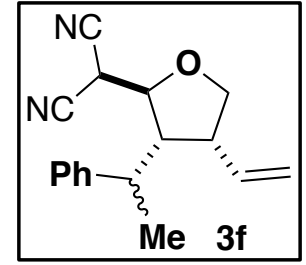




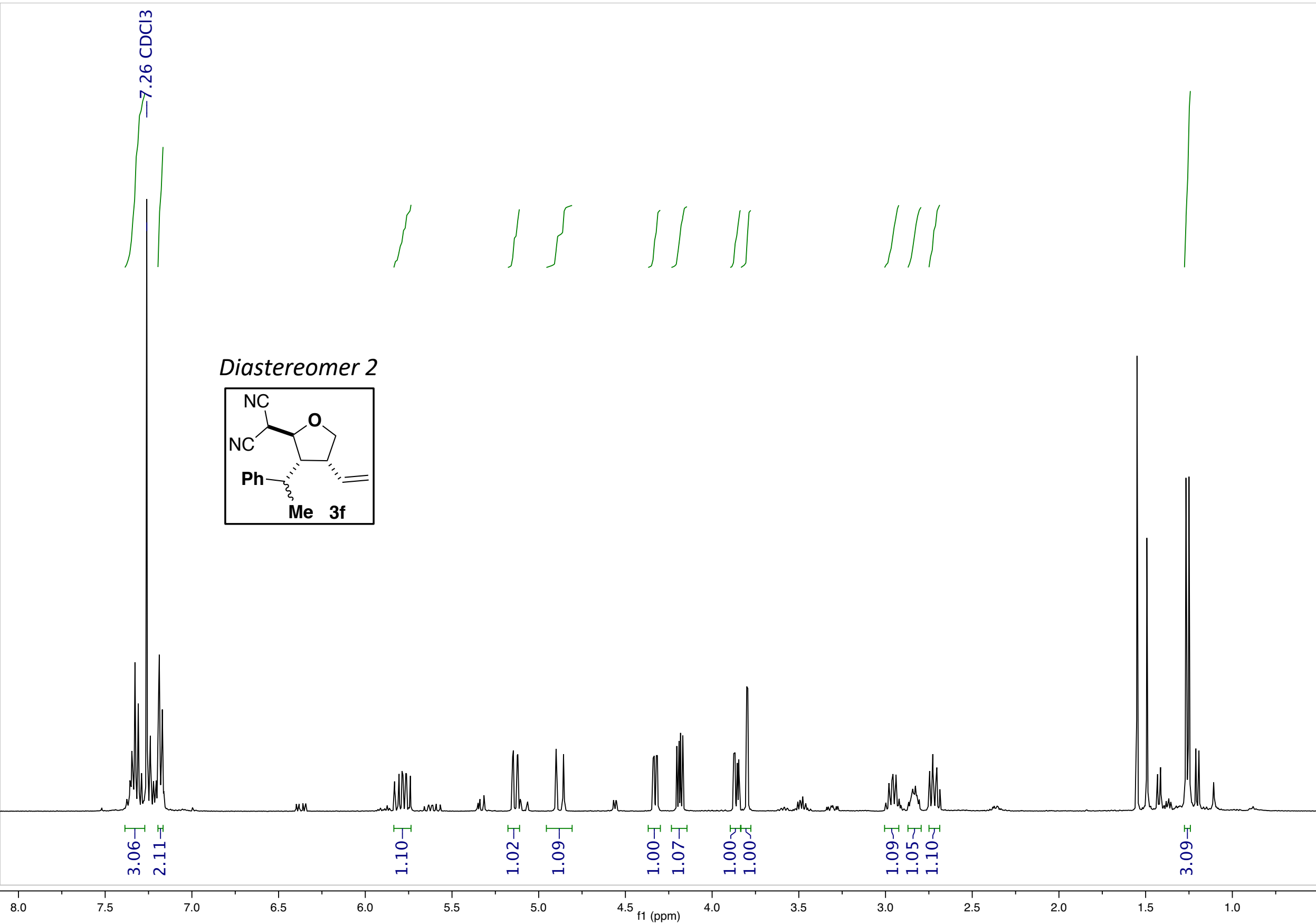




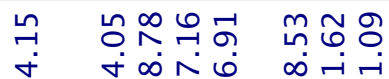

$$
\begin{aligned}
& \text { 方 }
\end{aligned}
$$

\section{Diastereomer 2}

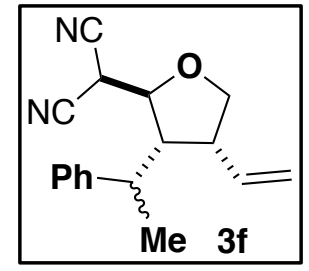

이 $m$

尺 

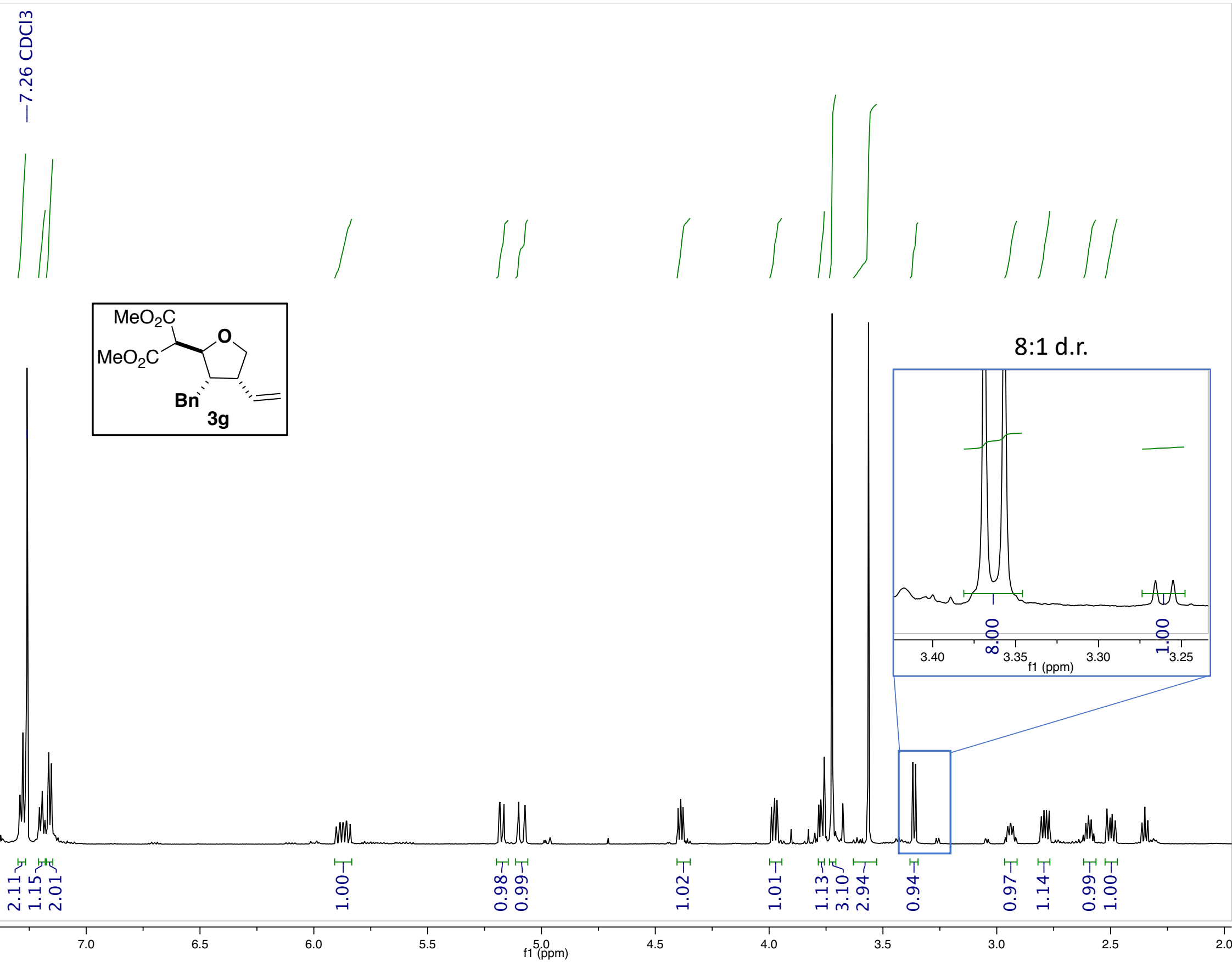


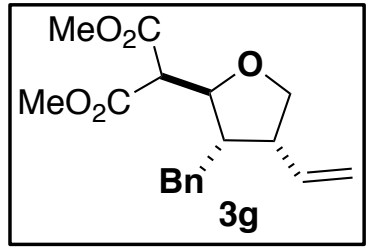

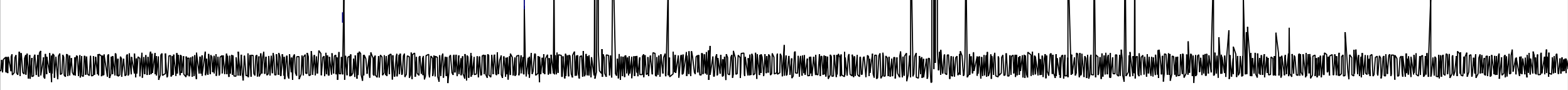

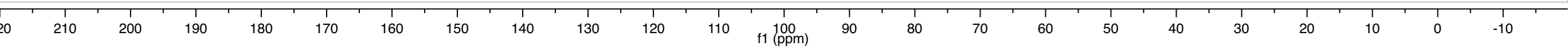



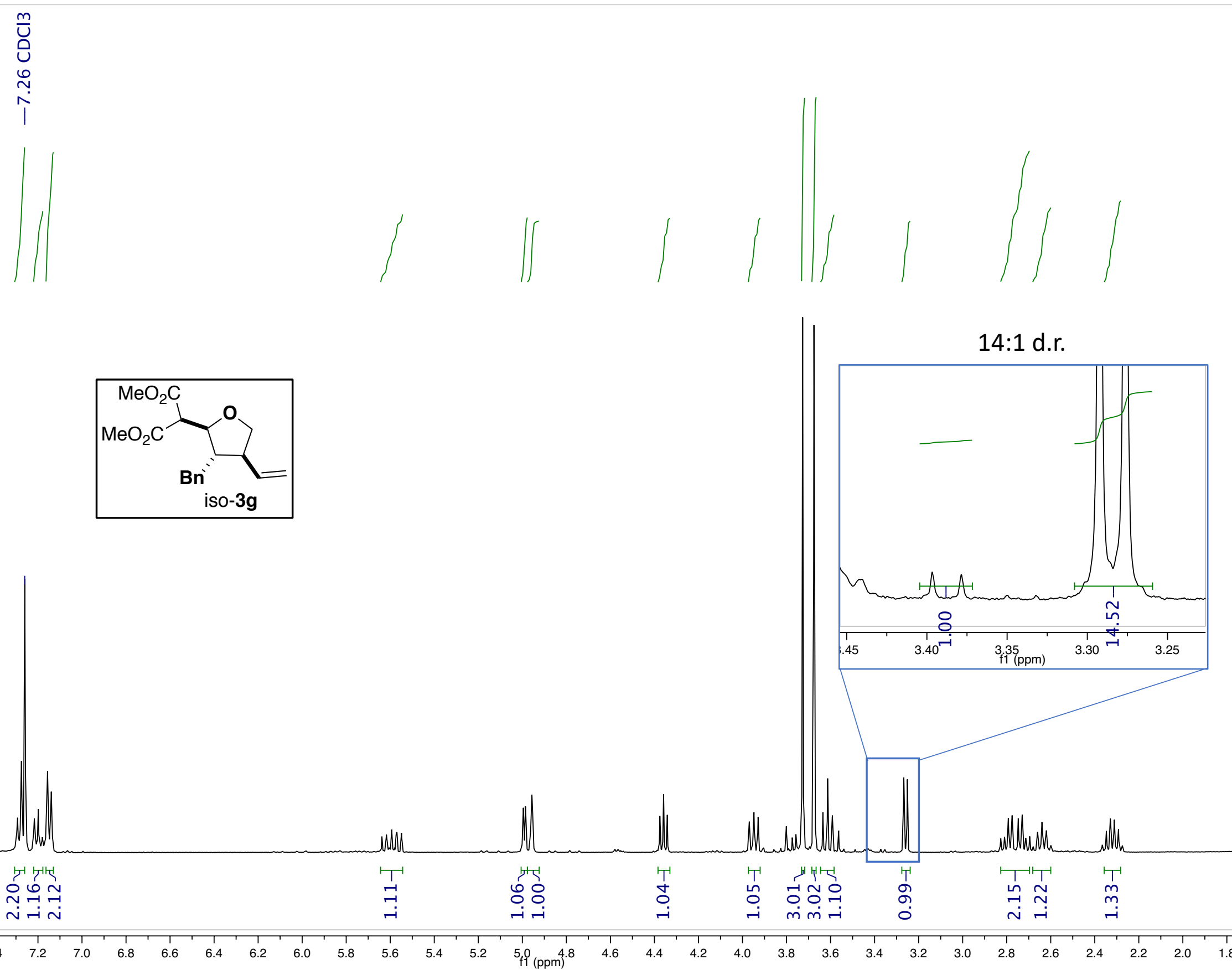


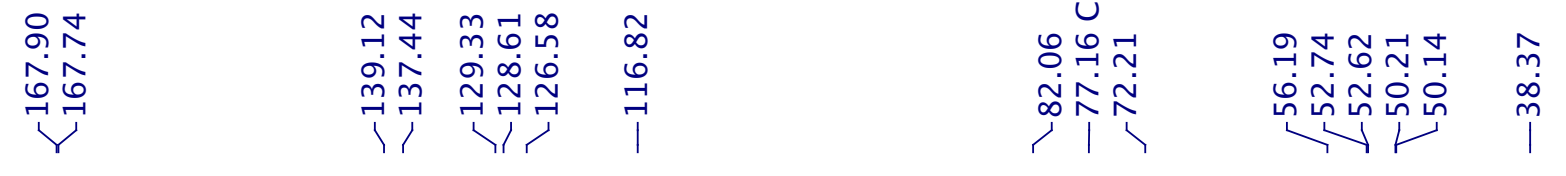

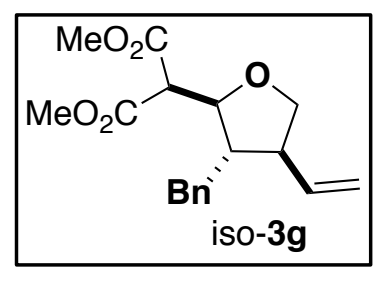

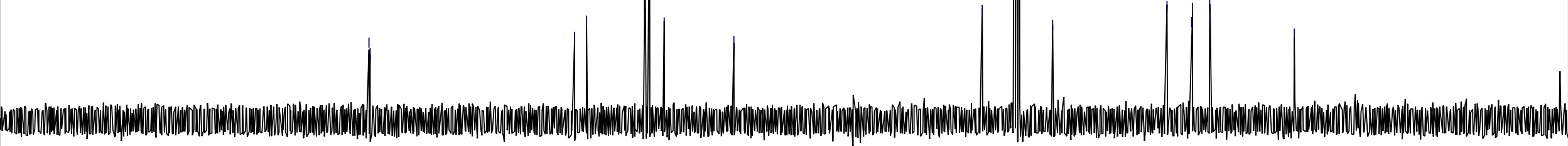

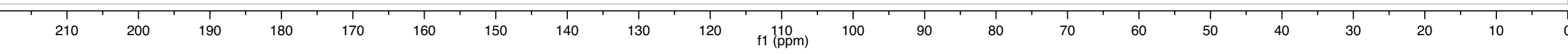



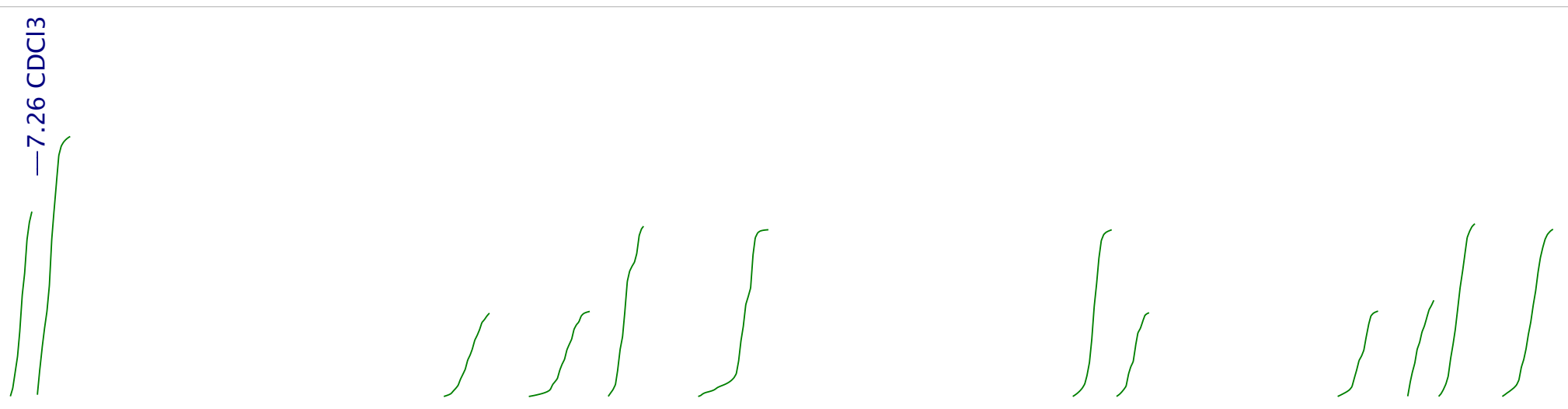

8:1 d.r.
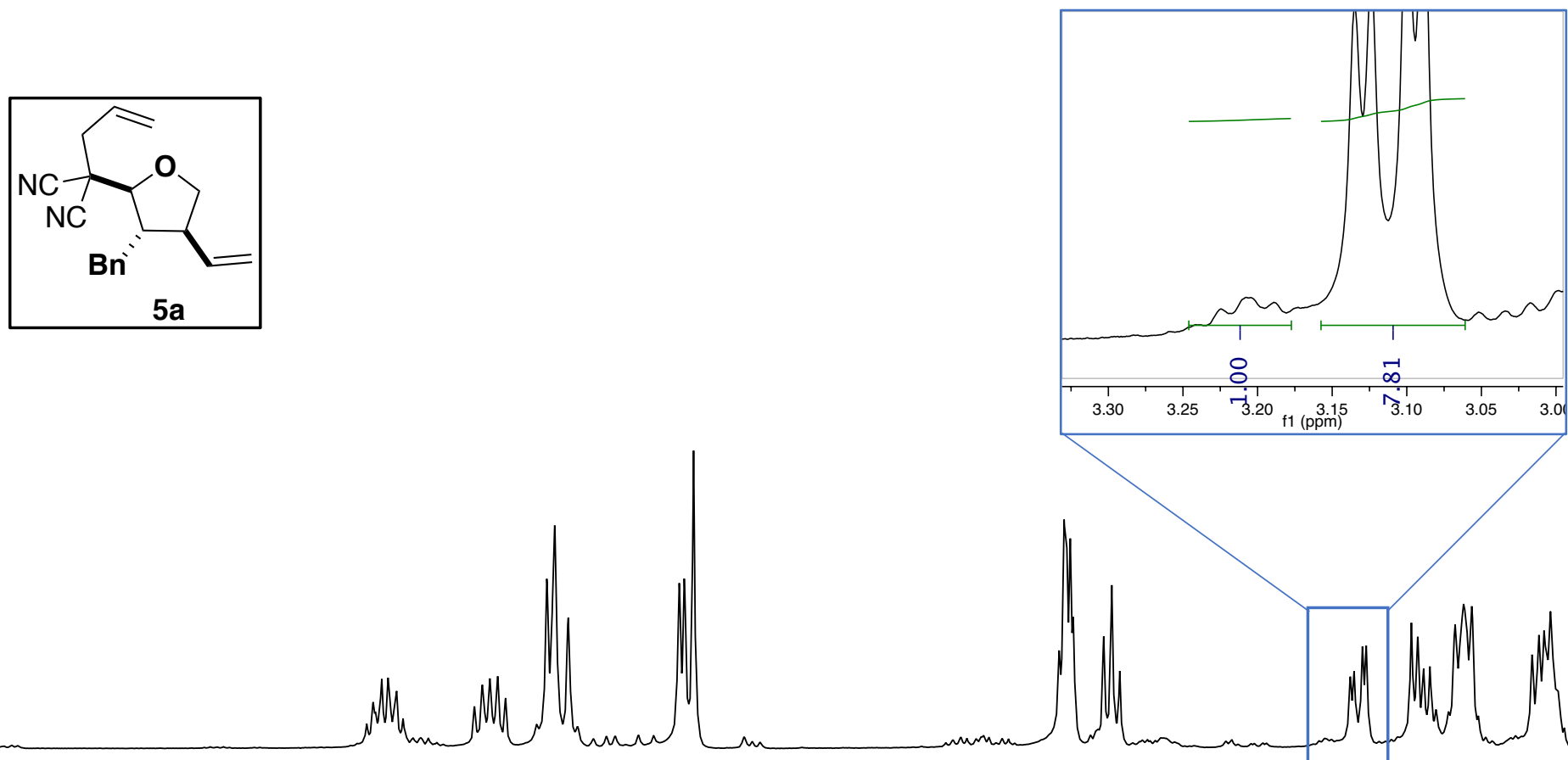

$\stackrel{\text { 눈 }}{\stackrel{1}{\sim}} \underset{\sim}{\sim}$

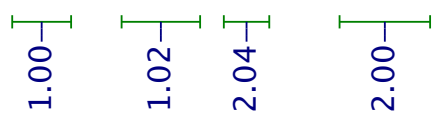

N

2 f1 $(\mathrm{ppm})$ 


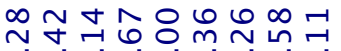

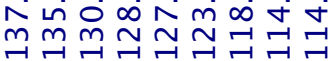

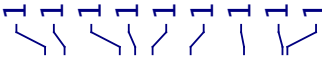

บับ

กิำำ

舟金
กินิํํำ

ㅇํํ भ $\cong \hat{m}$

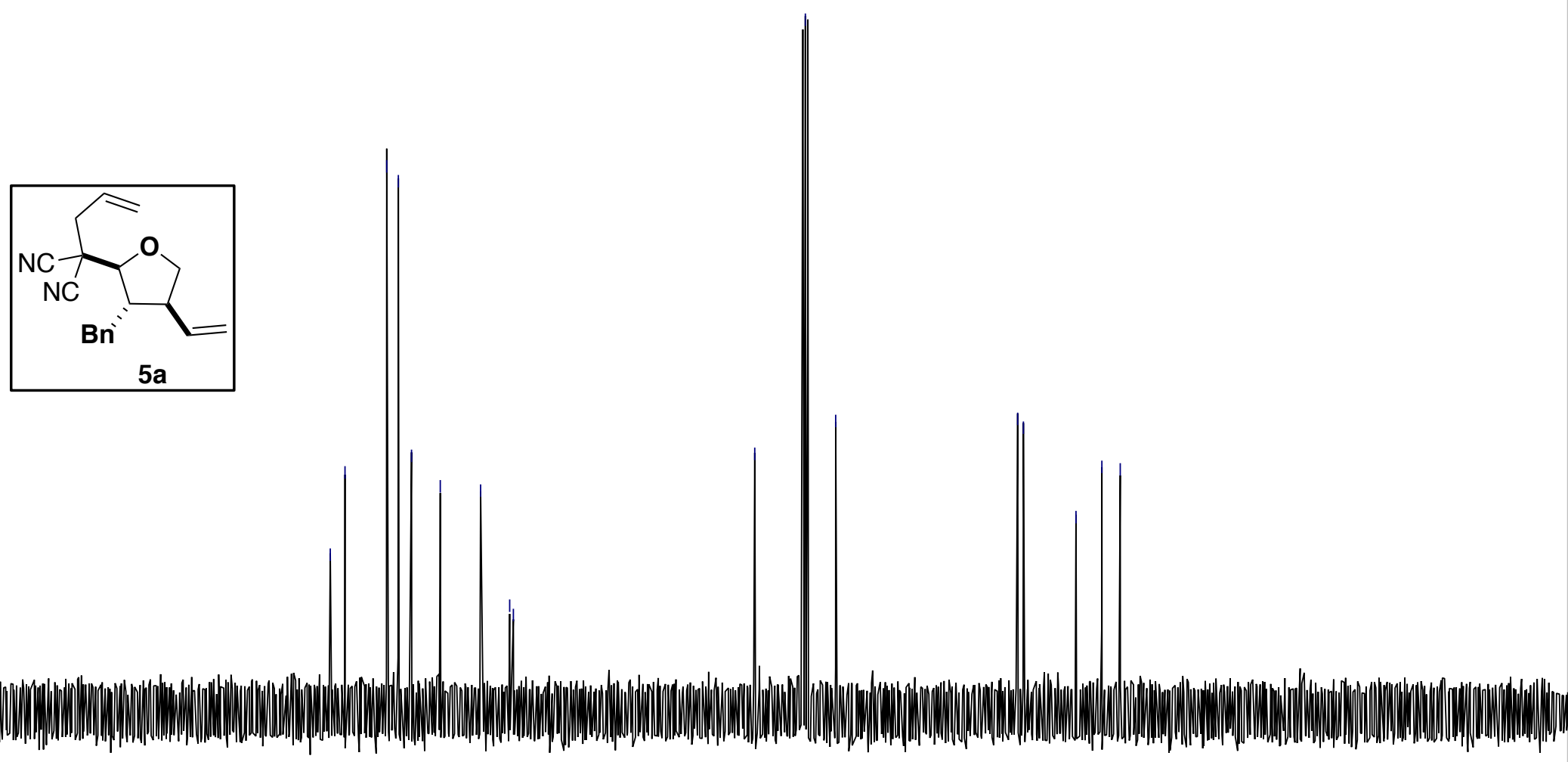




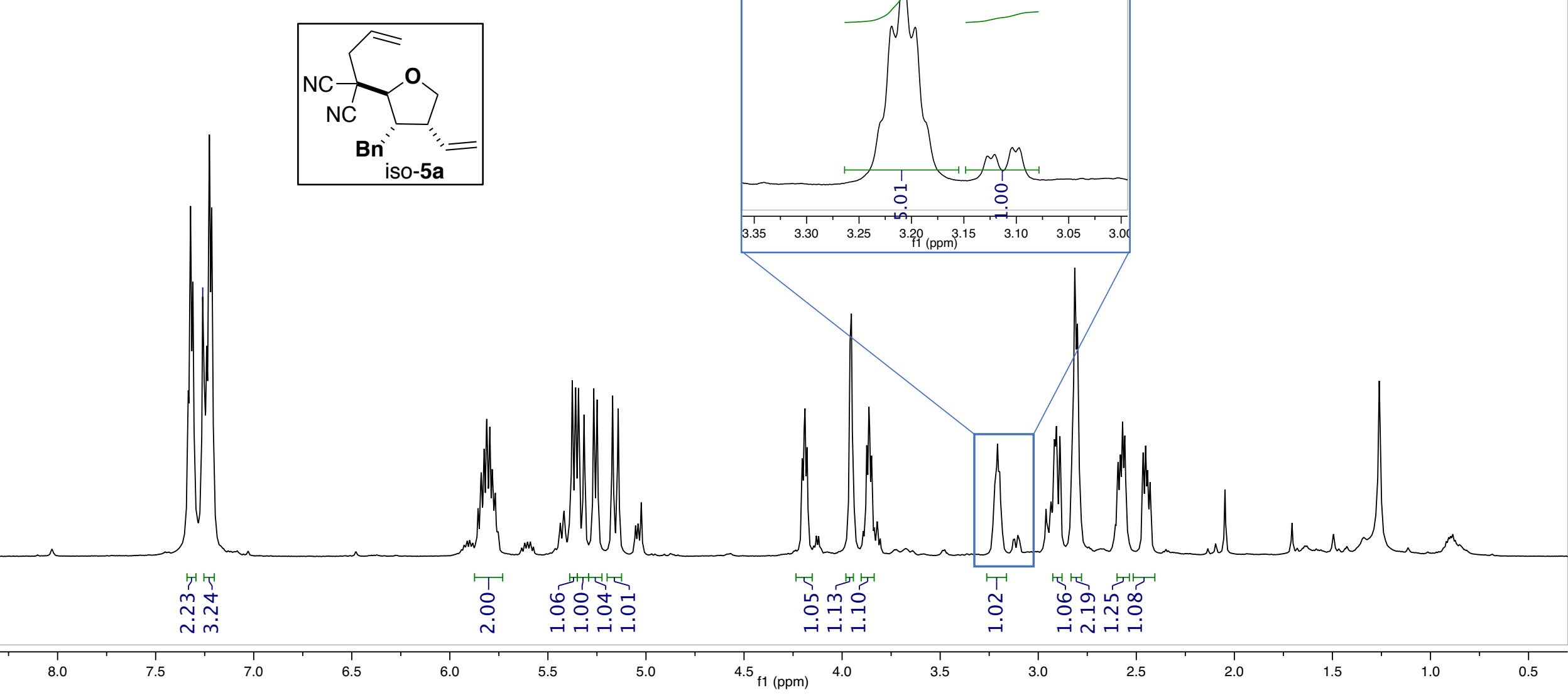

5:1 d.r. 


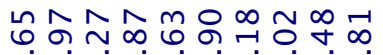

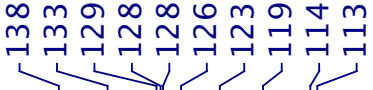
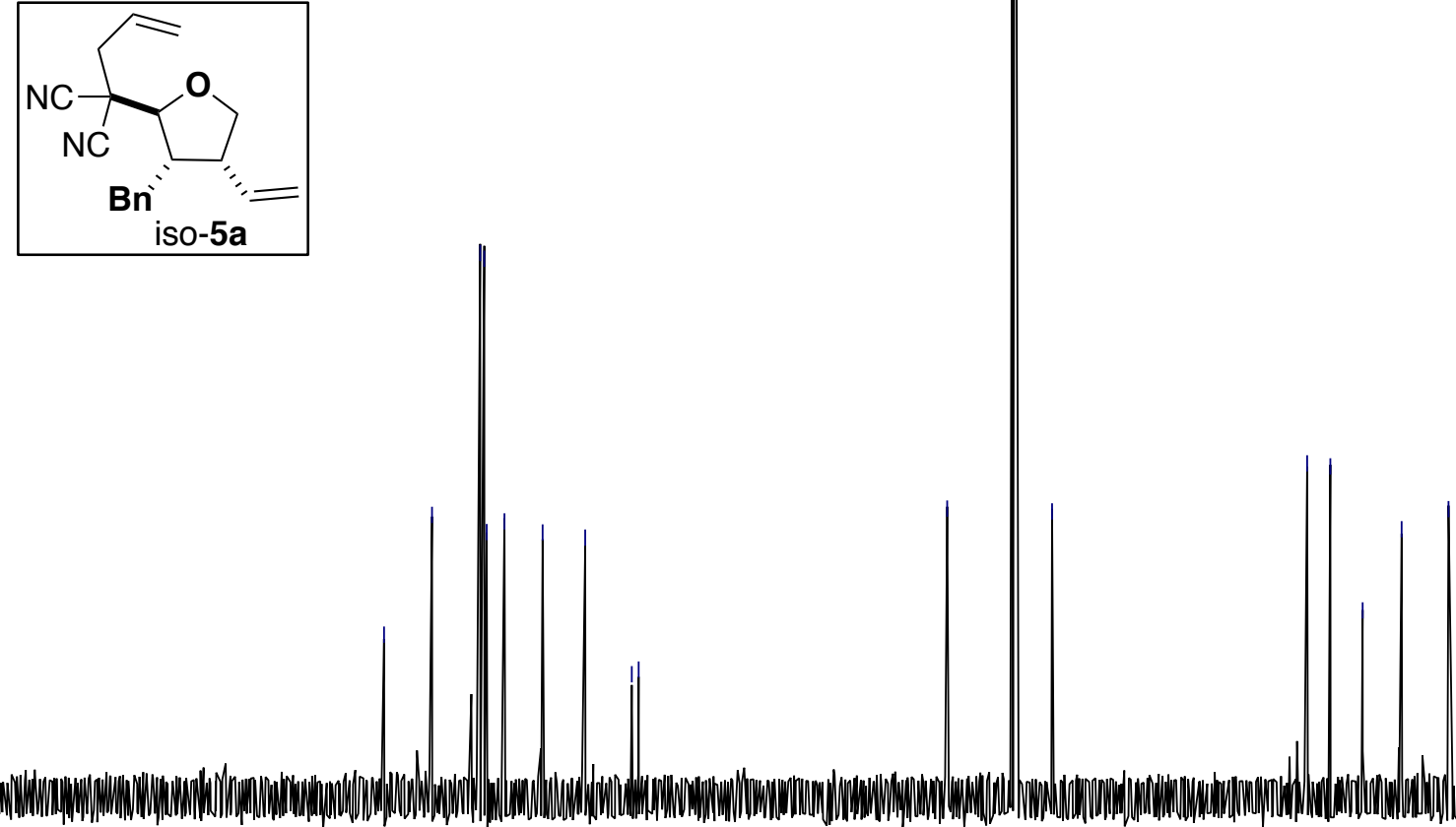

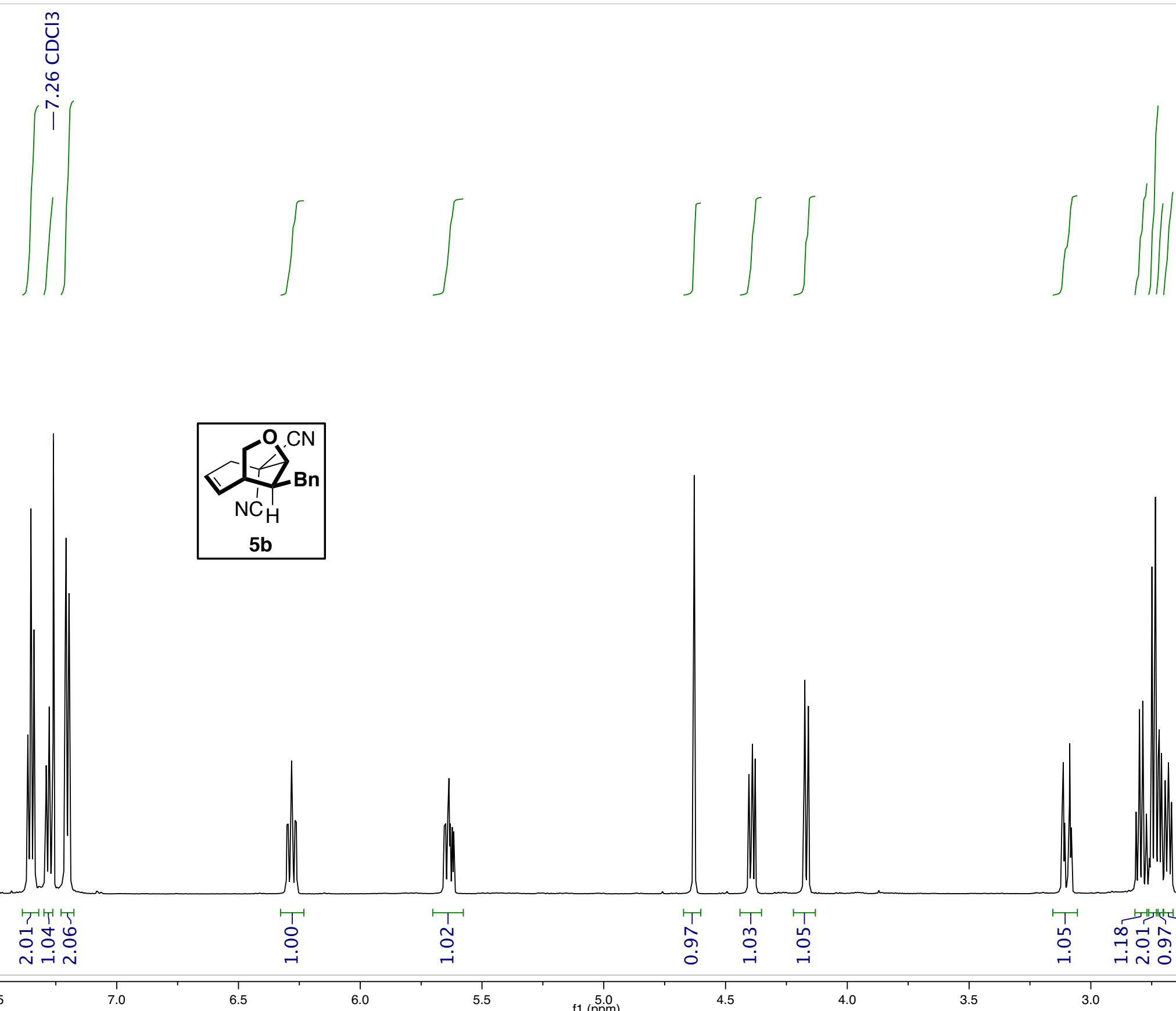

ग मान्म

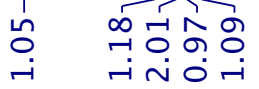

6.0

5.5

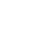




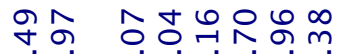

$$
\begin{aligned}
& \text { 京篎 }
\end{aligned}
$$
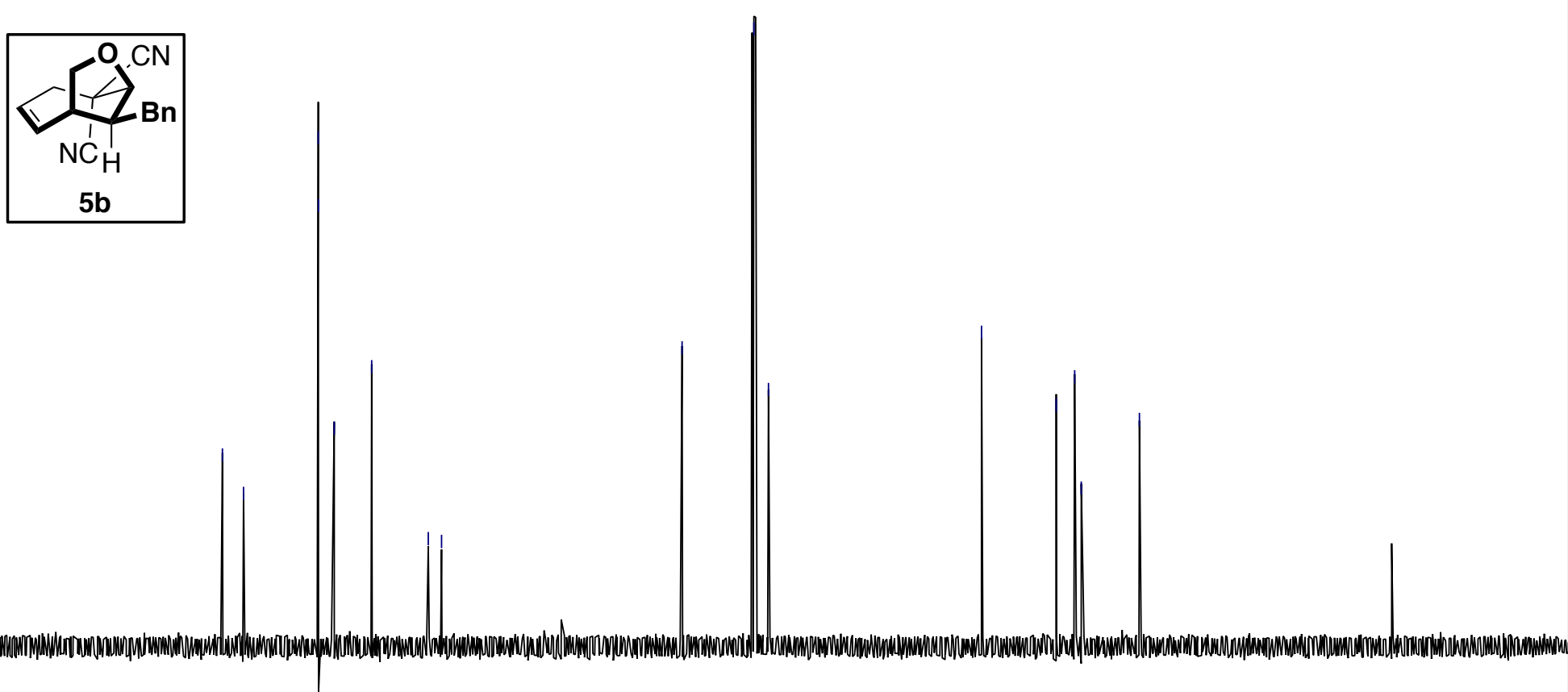


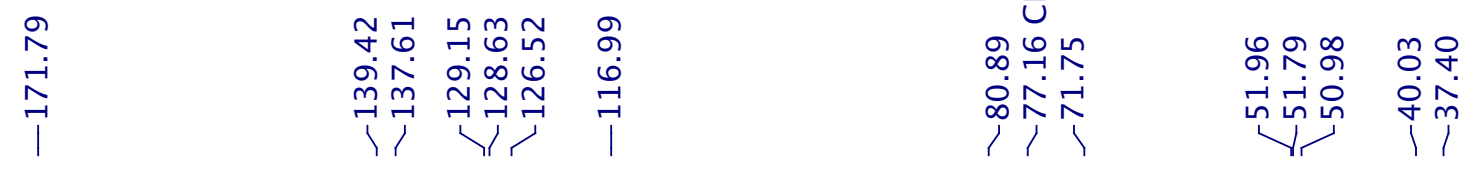

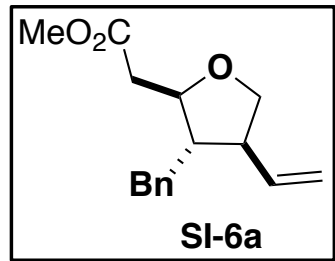

$210 \quad 200$

190 

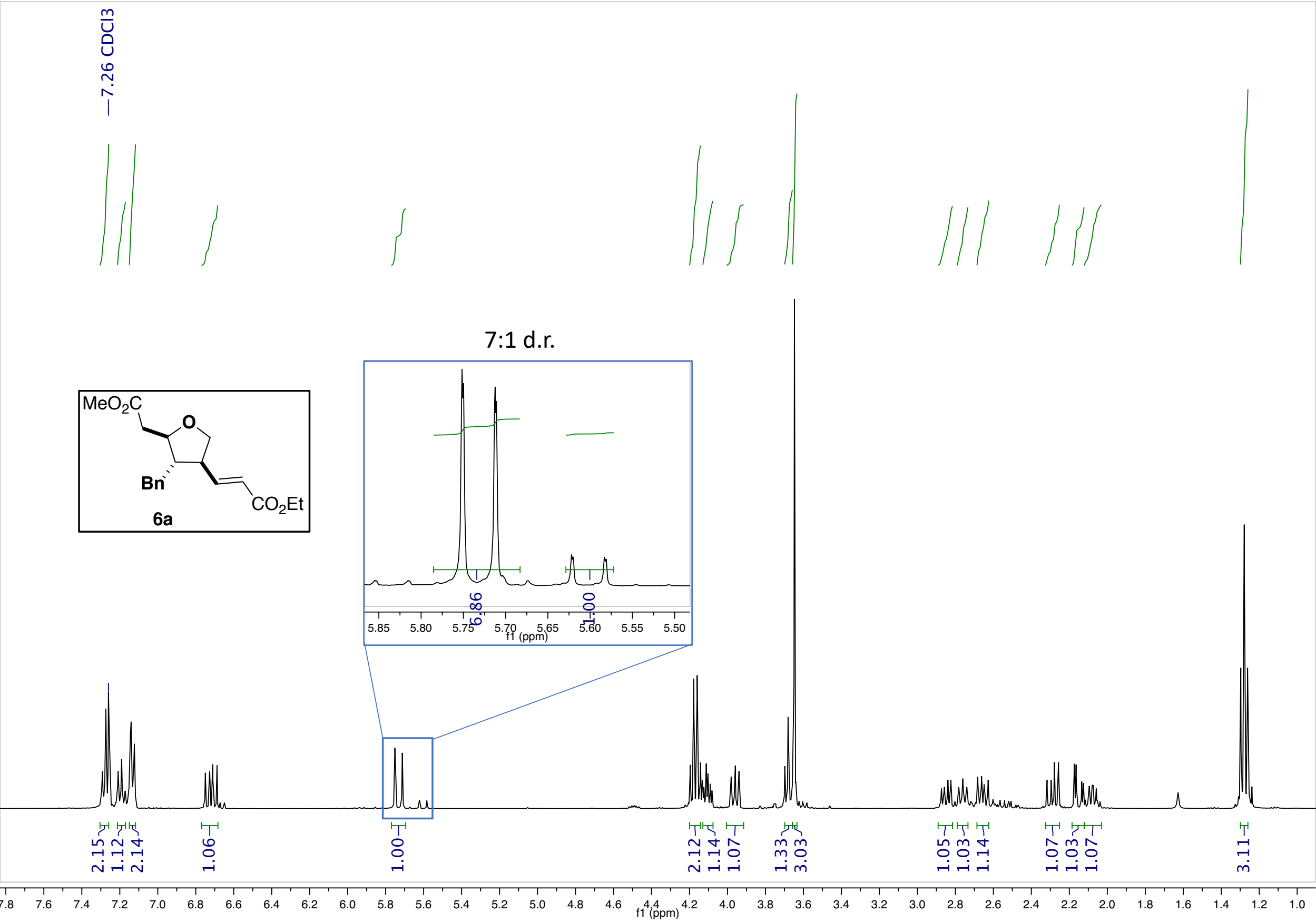

7:1 d.r. 


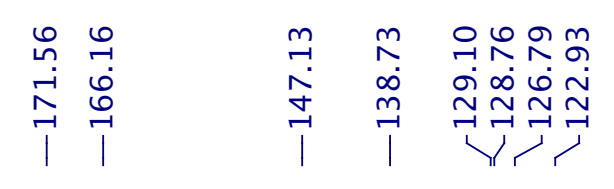

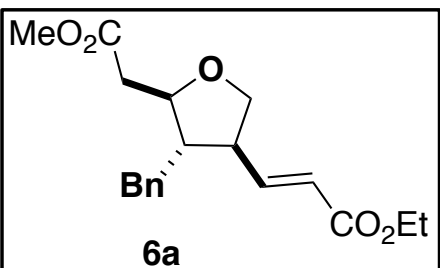




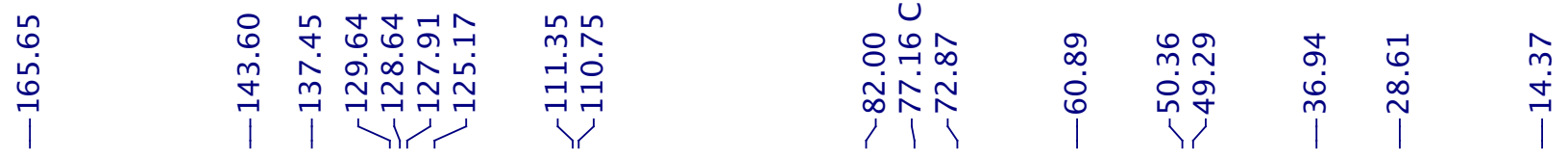

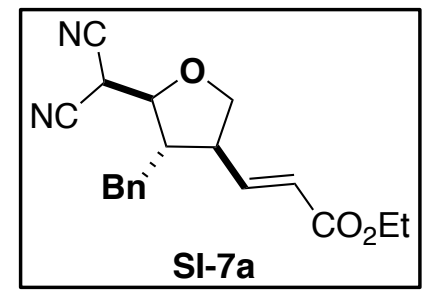

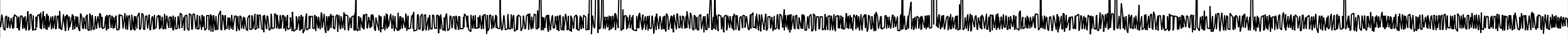

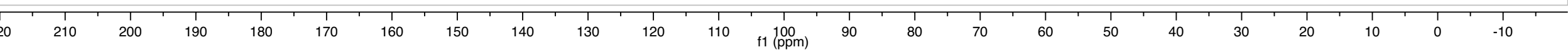


$\frac{m}{U}$
0
0

$i$
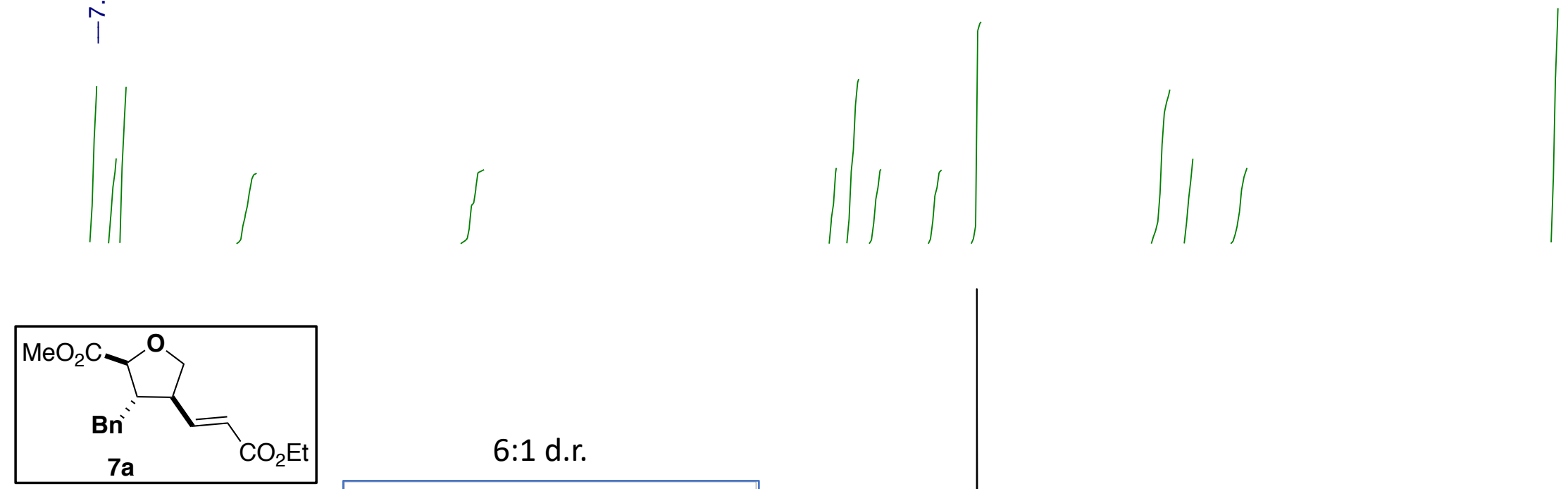

6:1 d.r.

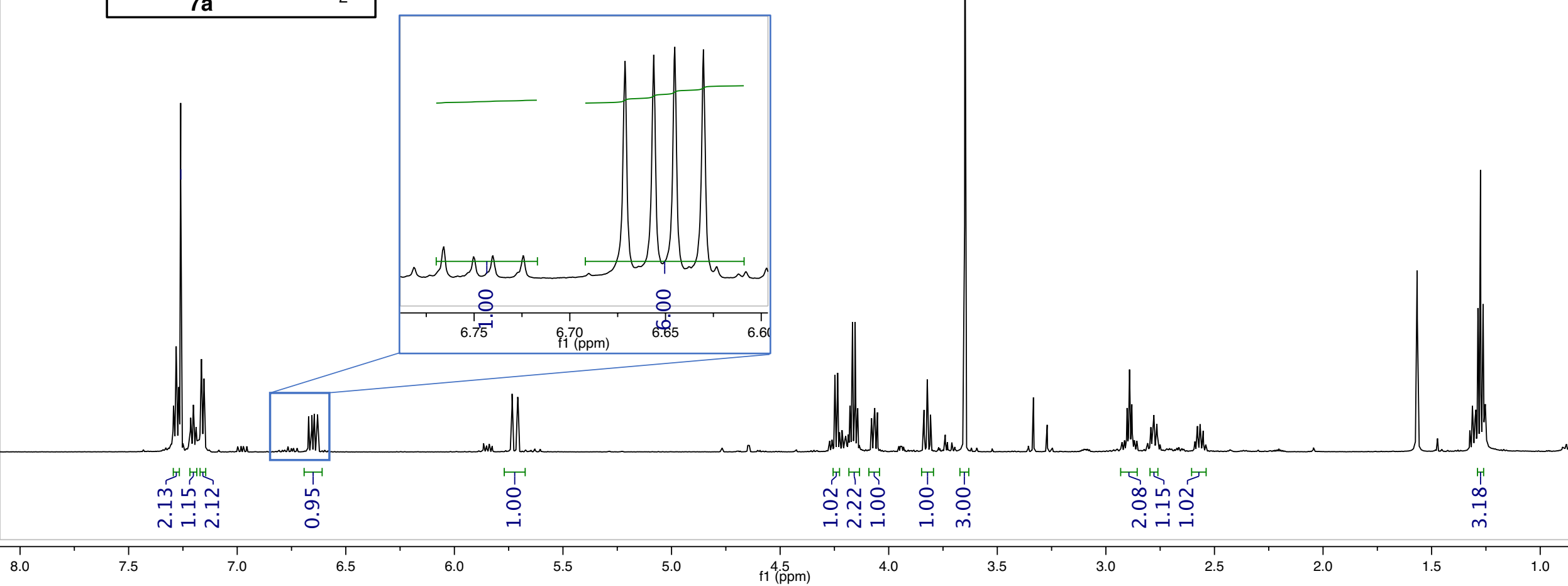




$$
\begin{aligned}
& \text { จ }
\end{aligned}
$$

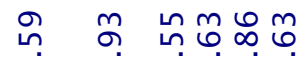

$$
\begin{aligned}
& \text { กิ } \\
& \text { 官 N }
\end{aligned}
$$
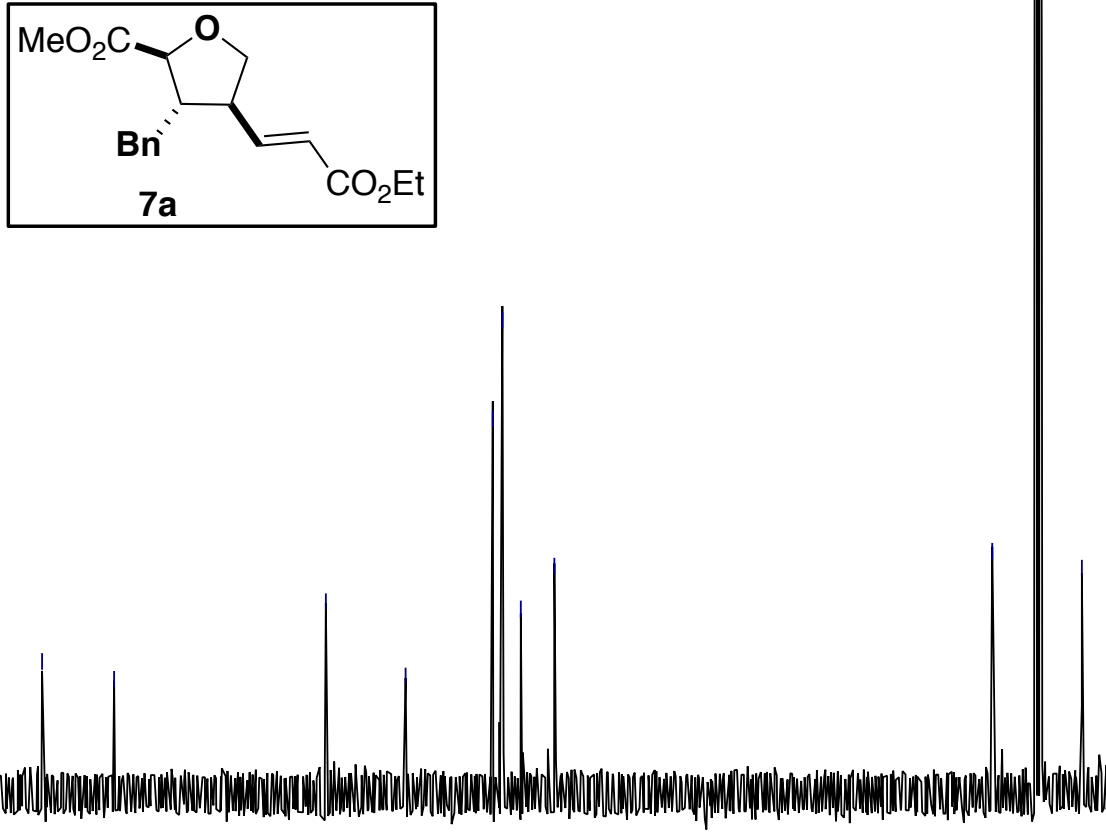
$\frac{m}{U}$
$\cup$
0
$\stackrel{0}{i}$
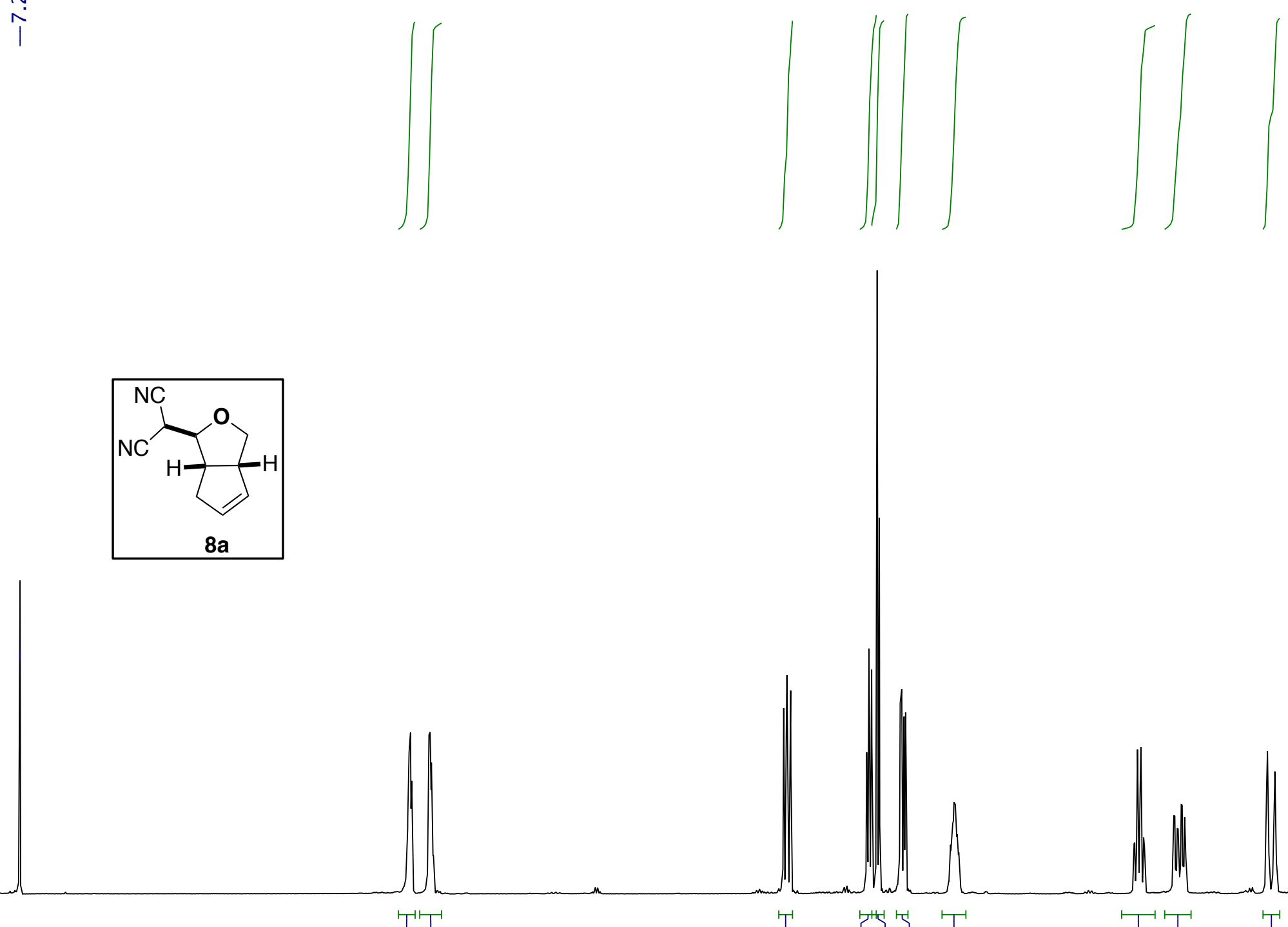

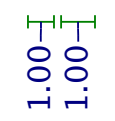

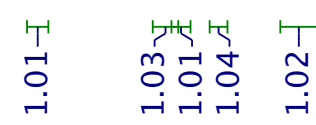

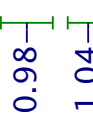

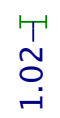

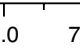

7.6

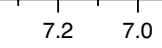

$\begin{array}{llll}6.8 & 6.6 & 6.4 & 6.2\end{array}$
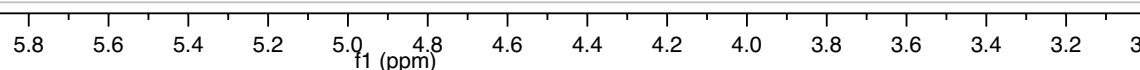


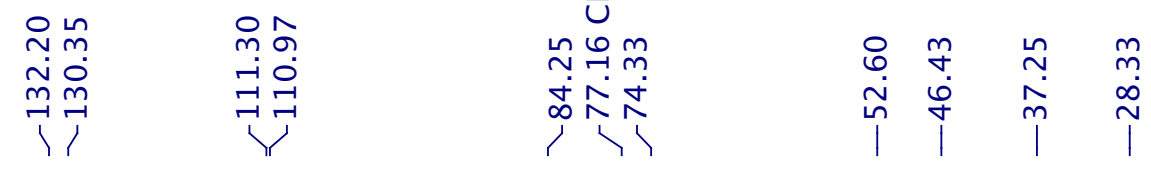

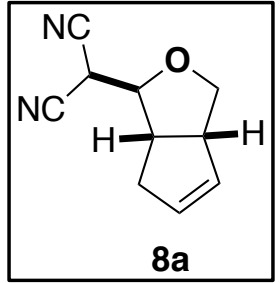

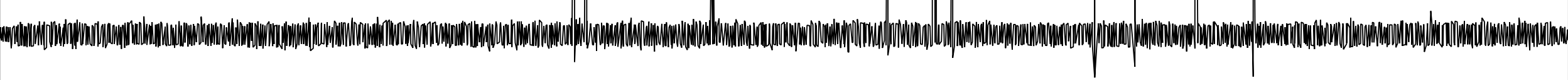

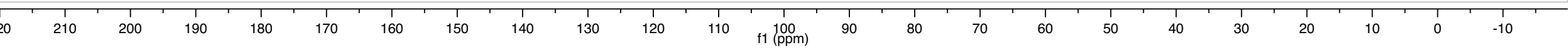



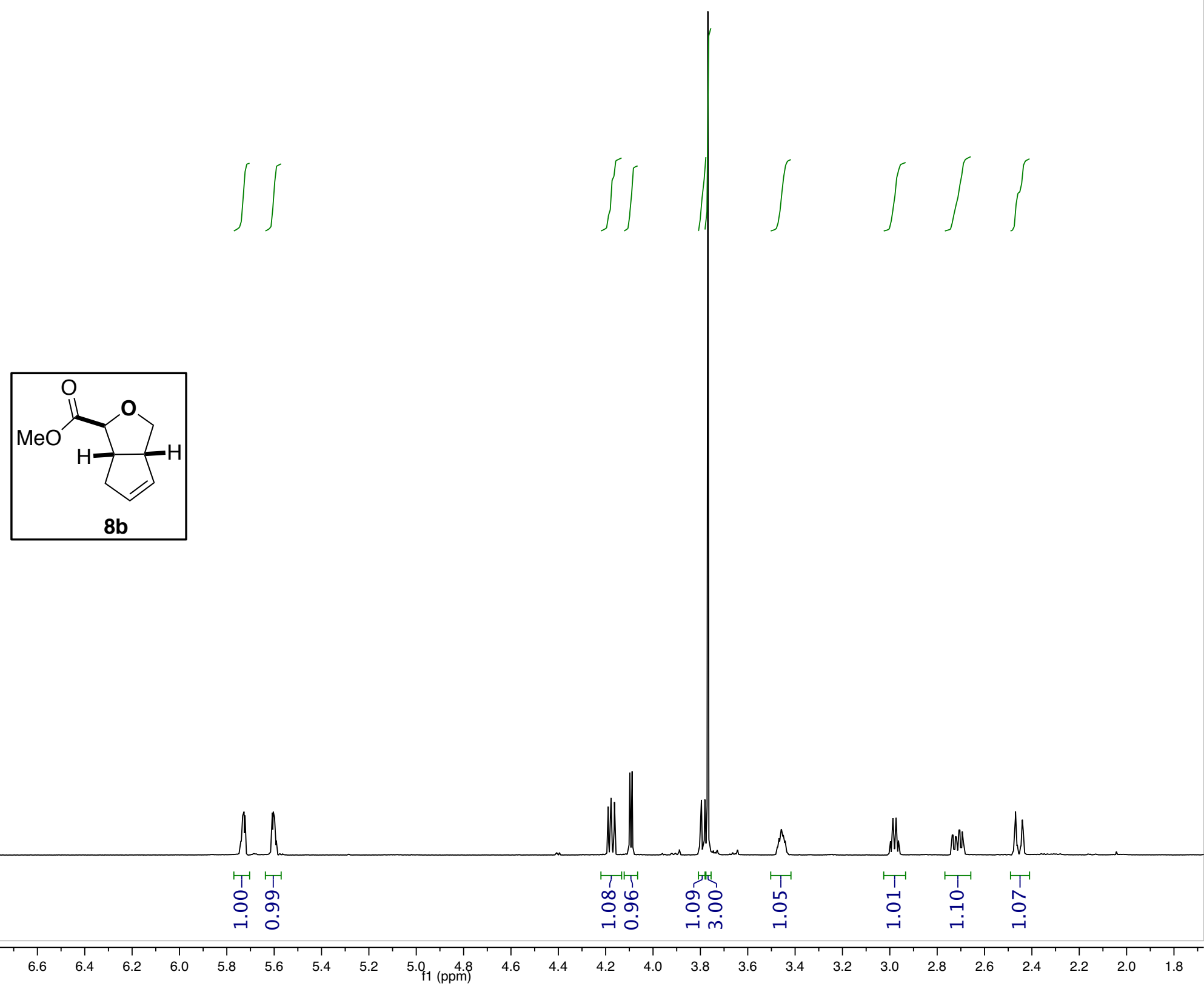
$\stackrel{\substack{n \\ m \\ n}}{n}$

ธั ธิ

min

出望

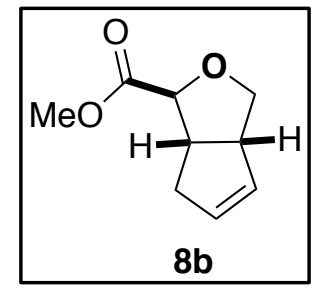

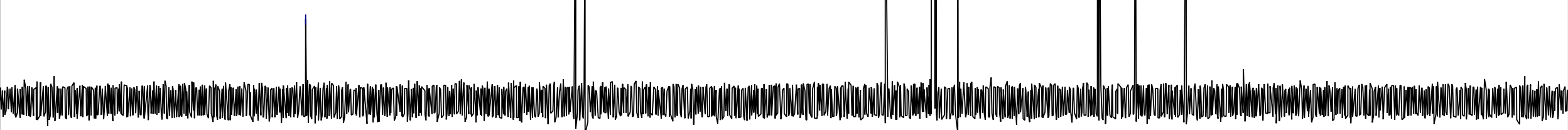
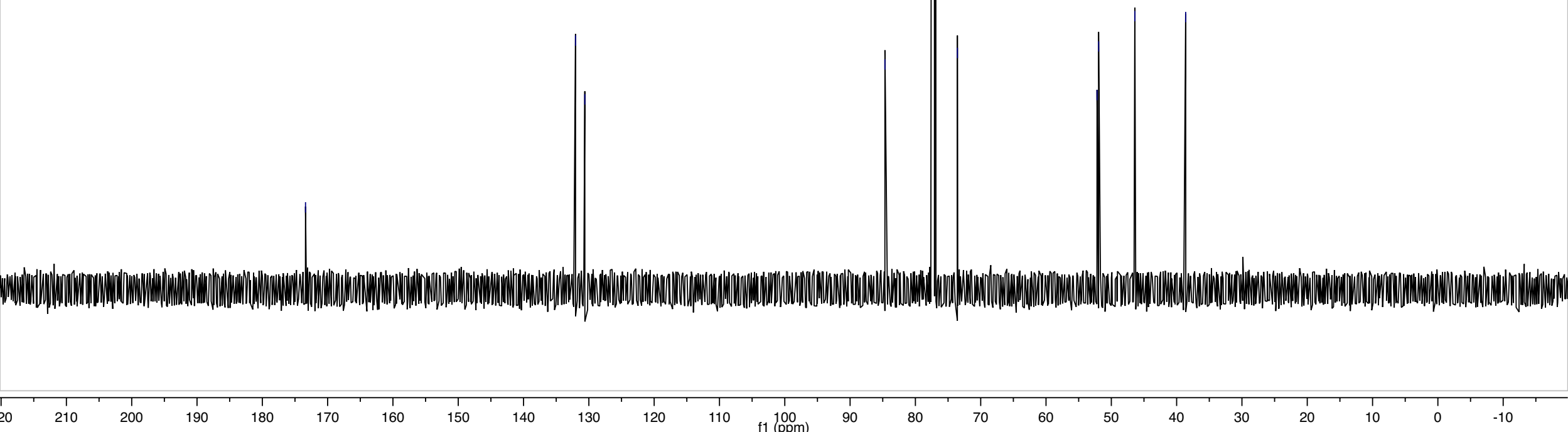